\title{
DETERMINATION OF THE 5 MW GROSS NOMINAL DESIGN CASE BINARY CYCLE FOR POWER GENERATION AT RAFT RIVER, IDAHO
}

\author{
I. J. INGVARSSON W. W. MADSEN
}

MASTER

December 1976

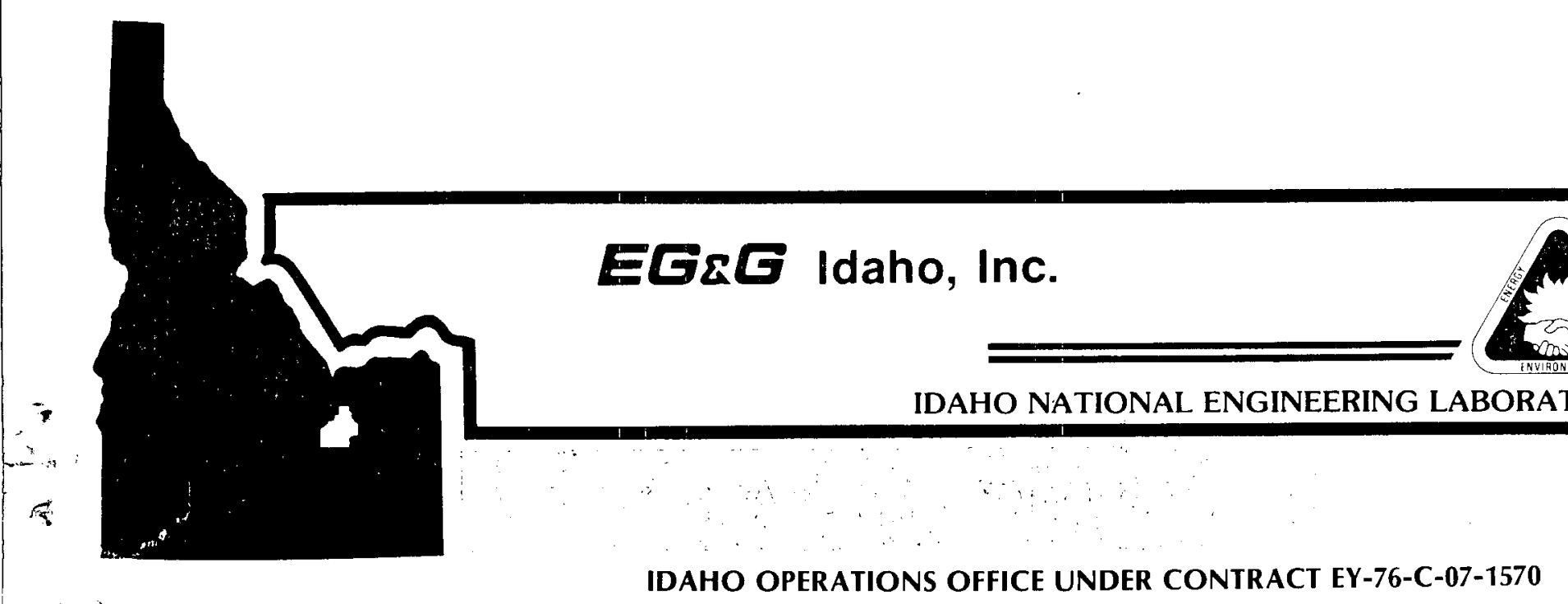




\section{DISCLAIMER}

This report was prepared as an account of work sponsored by an agency of the United States Government. Neither the United States Government nor any agency Thereof, nor any of their employees, makes any warranty, express or implied, or assumes any legal liability or responsibility for the accuracy, completeness, or usefulness of any information, apparatus, product, or process disclosed, or represents that its use would not infringe privately owned rights. Reference herein to any specific commercial product, process, or service by trade name, trademark, manufacturer, or otherwise does not necessarily constitute or imply its endorsement, recommendation, or favoring by the United States Government or any agency thereof. The views and opinions of authors expressed herein do not necessarily state or reflect those of the United States Government or any agency thereof. 


\section{DISCLAIMER}

Portions of this document may be illegible in electronic image products. Images are produced from the best available original document. 
Printed in the United States of America Available from

National Technical Information Service U.S. Department of Commerce 5285 Port Royal Road

Springfield, Virginia 22161

Price: Printed Copy $\$ 6.00$; Microfiche $\$ 3.00$

\section{NOTICE}

This report was prepared as an account of work sponsored by the United States Government. Neither the United States nor the Energy Research and Development Administration, nor the Nuclear Regulatory Commission, nor any of their employees, nor any of their contractors, subcontractors, or their employees, makes any warranty, express or implied, or assumes any legal liability or responsibility for the accuracy, completeness or usefulness of any information, apparatus, product or process disclosed, or represents that its use would not infringe privately owned rights. 
DETERMINATION OF THE 5 MW GROSS NOMINAL DESIGN

CASE BINARY CYCLE FOR POWER GENERATION AT RAFT RIVER, IDAHO

EG\&G Idaho, Inc.

IDAHO NATIONAL ENGINEERING LABORATORY

Idaho Falls, Idaho 83401

Edit by

I. J. Ingvarsson

W. W. Madsen

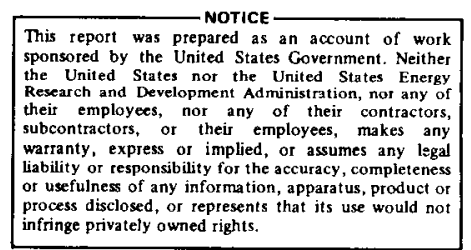

infringe privately owned rights.

Date Published - December 1976

Prepared for the

ENERGY RESEARCH AND DEVELOPMENT ADMINISTRATION

DIVISION OF GEOTHERMAL ENERGY AND IDAHO OPERATIONS OFFICE

Under Contract No. EY-76-C-07-1570 


\begin{abstract}
$\underline{\text { ABSTRACT }}$
This report documents a series of Rankine Cycle studies for power generation utilizing geothermal fluid as the heat source and isobutane as the working fluid. To find the plant configuration which would most effectively utilize the available energy, a parametric study was performed. The study considered the desirability of supercritical, single boiler or double boiler cycles, and the relative boiler temperatures and percentage isobutane flow split between the boilers in the double cycles for geothermal fluid temperatures of $260^{\circ} \mathrm{F}$ to $360^{\circ} \mathrm{F}$. This study was designed to discover thermodynamic trends which would point to an optimum isobutane cycle for geothermal fluid temperatures in this temperature range. The results of the parametric study were applied to derive a Nominal Design Case for a demonstration plant at Raft River, with a geothermal fluid resource at $290^{\circ} \mathrm{F}$. In addition, plant variations due to tolerances applied to thermodynamic properties and other key factors are included.
\end{abstract}




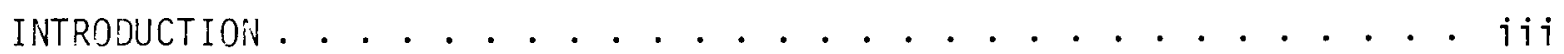

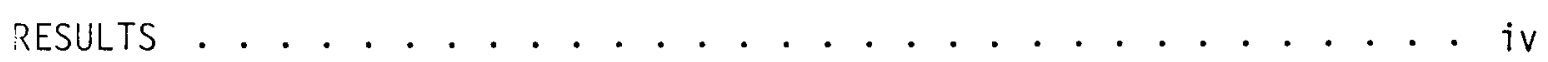

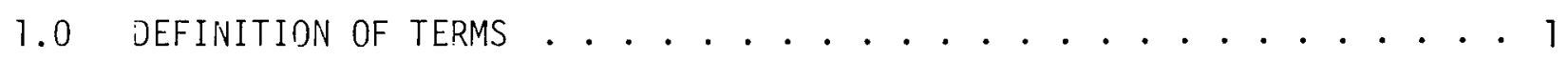

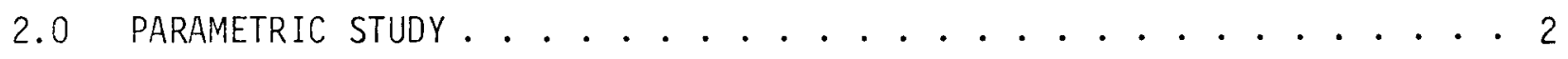

2.1 ASSUMPTIONS USED FOR THE PARAMETRIC STUDY OF THE GEOTHERMAL

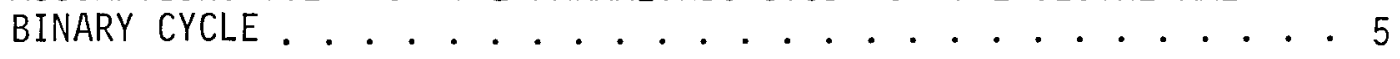

2.2 MEDIUM TEMPERATURE BRINE CASES . . . . . . . . . . . . 7

2.2.1 Single Boiler Cycles.............. . . 7

2.2.2 Double Boiler Cycles............. . 7

2.2.3 Results of the Medium Temperature Brine Cases . . . . 16

2.3 HIGH TEMPERATURE BRINE CASES . . . . . . . . . . 30

2.3.1 Single Boiler Cycles........... . . 30

2.3.2 Supercritical Cycles............ . . 33

2.3.3 Double Boiler Cycles . . . . . . . . . . 37

2.3.4 Results of the High Temperature Brine Cases . . . . . 45

2.4 RESULTS OF THE PARAMETRIC STUDY . . . . . . . . . . . . 49

3.0 DETERMINATION DF THE NOMINAL DESIGN CASE........... 53

4.0 TOLERANCE STUdY OF THE NOMINAL DESIGIN CASE . . . . . . . . . 55

4.1 BRINE INLET TEMPERATURE ................. 56

4.2 CONDENSING TeMPERATURE . . . . . . . . . . 57

4.3 GEOTHERMAL FLUID FOULING FACTORS . . . . . . . . . 58

4.4 ISOBUTANE SPECIFIC HEATS . . . . . . . . . . . 60

4.5 RESULTS OF THE TOLERANCE STUDY ................ 61

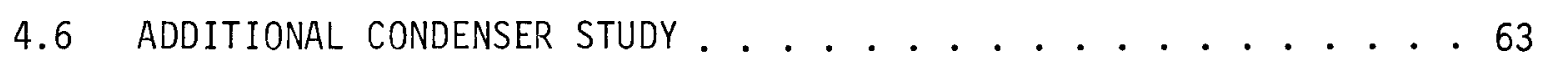


5.0 PLANT COST STUDIES . . . . . . . . . . . . . . . 67

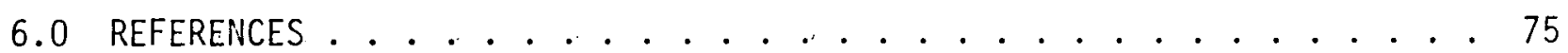

APPENDIX A - GEØSYS DOCUMENTATION

PROGRAM MODIVICATIONS ................... . . . . .

INPUT TO THE PROGRAM . . . . . . . . . . . . . . . . . . A-8

PROGRAM LISTING AND SAMPLE RUN . . . . . . . . . . . A-12

APPENDIX B - COST EQLATION DERIVATIONS 
INTRODUCTION

This report documents a series of Rankine Cycle studies for power generation utilizing geothermal fluid as the heat source and isobutane as the working fluid. The end purpose of this study was to determine the nominal configuration and steady state operation characteristics for a $5 \mathrm{MW}$ gross plant operating from the Raft River Idaho Geothermal Resource. To find the plant configuration which would most effectively utilize the available energy, a parametric study was performed. The study considered the desirability of supercritical, single boiler or double boiler cycles, and the relative boiler temperatures and percentage isobutane flow split between the boilers in the double boiler cycles for geothermal fluid temperatures of $260^{\circ} \mathrm{F}$ to $3600 \mathrm{~F}$. This study was designed to discover thermodynamic trends which would Doint to an optimum isobutane cycle for geothermal fluid temperatures in this temperature range. Optimum was defined as the case which utilized the greatest amount of available geothermal fluid energy, and was indexed as a variable called effectiveness (net power output per pound of geothermal fluid circulated through the plant). The results of the parametric study were applied to derive a Nominal Design Case for a demonstration plant at Raft River, with a geothermal fluid resource at $290^{\circ} \mathrm{F}$. 


\section{RESULTS}

The Nominal Design Case was defined for the Raft River geothermal fluid resource at $2900^{\circ} \mathrm{F}$. The optimum combination for that temnerature is a cycle with two boilers operating at $240^{\circ} \mathrm{F}$ and $180^{\circ} \mathrm{F}$ with the isobutane flow split so that there is approximately twice as much flow going through the high pressure boiler as through the low pressure boiler. The effectiveness for this system is 3.2 watt - hrs/lb of geothermal fluid. The net thermal efficiency is $7.5 \%$. Parasitic losses, including geothermal fluid supply pumping and reinjection, are on the order of one third of the gross power. Thus, the Raft River plant, which is to be rated at $5 \mathrm{MW}$ gross electrical power output requires about 40 MW thermal input, will produce a net power of $3.35 \mathrm{MW}(e)$, and requires approximately $1 \times 10^{6} \mathrm{lb} / \mathrm{hr}$ of geothermal fluid. The parametric study yielded several thermodynamic behavioral patterns related to the binary Rankine Cycle. These are summarized in Section 2.4 and discussed more fully in Sections 2.2 and 2.3. 


\subsection{Definition of Terms}

Brine: A short term used to refer to the geothermal fluid. This usage does not imply that the geothermal fluid has a significant saline content; it is only used for abbreviation and convenience.

Effectiveness: The effectiveness is a measure of brine utilization. It is defined as the net power output of the plant per pound of brine circulated (Btu/lbm brine). The brine wells are a major plant cost, therefore it is imnortant to maximize the effectiveness, resulting in a minimum brine flow rate.

Flow Split: (WF2/WF1) For a two boiler system, the flow split is the ratio of the mass flow in the low pressure (LP) path to the flow in the high pressure (HP) path. A flow split of 0.0 indicates a single boiler system. Pinch Point: The pinch point is a hardware - based parameter which is the minimum difference between the isobutane temperature and the brine temperature in the system. It occurs at the brine outlet of the boiler. Pinch Point Control: In a two-boiler system, the pinch points and the flow split are closely related parameters. The minimum pinch points for both boilers are input, as well as the flow split. There is a unique flow split value at which both pinch points are minimized. For any other flow split, one of the pincli points will exceed the minimum value. The pinch point which is minimized is said to control, because it is from this minimum temperature difference that the brine flow requirement is calculated. Raft River: Refers to the location of the geothermal resource in the Raft River Valley about 15 miles south of Malta, Idaho. 


\subsection{Parametric Study}

In order to determine the boiler temperature(s) for a geothermal isobutane binary cycle, at which brine utilization is the highest, a parametric study was performed, using a modified version of the computer program GEgSYS. (1) The basic model was documented in Reference 1, and current modifications are documented in Appendix $A$ of this report. The GEDSYS model used is shown schematically in Figure 2.0-1 and consists of two boilers, two preheaters, two turbines, a condenser and severa 1 pumps.

The parametric study considered single boiler cases and combinations of boiler temperatures and flow solits in two boiler cases for high and medium temperature brine. Brine temperature was varied in $20^{\circ} \mathrm{F}$ increments from $260^{\circ} \mathrm{F}$ to $360^{\circ} \mathrm{F}$ and included a $290^{\circ} \mathrm{F}$ case corresponding to brine temperatures encountered at Raft River. For brine temperatures of $260^{\circ} \mathrm{F}$ to $300^{\circ} \mathrm{F}$, several high pressure (HP) boiler temperatures were chosen, and for each of these several low pressure (LP) boiler temperatures were chosen. Then for each combination of boiler temperatures, the flow split was varied from zero (single boiler case) to a value at which the effectiveness reached its peak. For the high temperature brine cases, $320^{\circ} \mathrm{F}$ to $360^{\circ} \mathrm{F}$, supercritical cycles were run in addition to the boiling cycles described above.

For all cases, the condenser temperature, and therefore turbine back pressure, were fixed at a value previously determined by cooling tower capabilities and atmospheric conditions.

Superheat and regeneration were not considered in this study for two reasons: First, the main purpose of this plant is to gain experience with various operating modes so that larger power plants may be designed with more certainty. Therefore, the plant must be able to produce power, and at the same time, be flexible enough to allow experimental operating procedures and hardware. Superheaters and regenerators are not essential components 


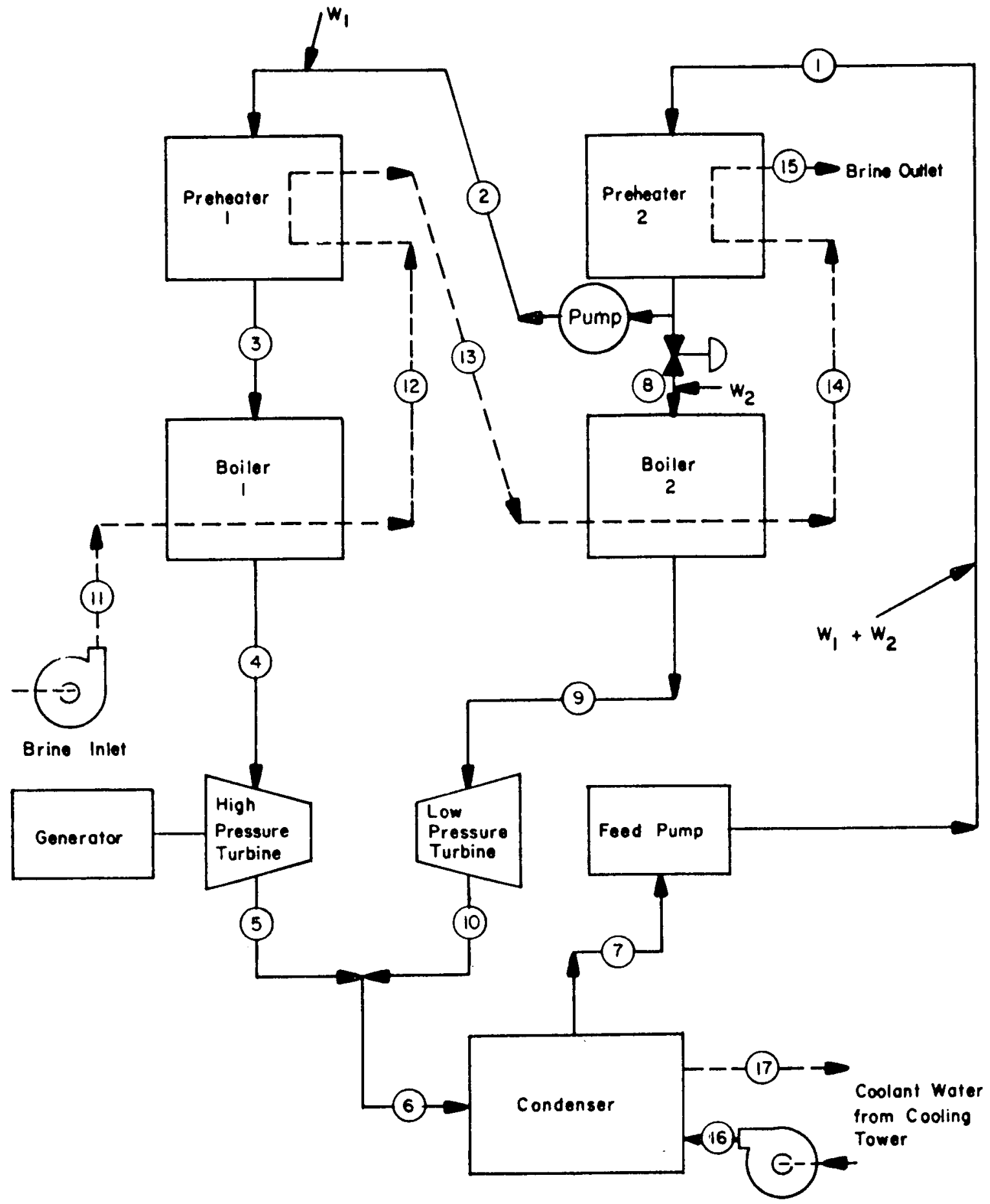

Figure 2.0-1 Double Boiling Isobutane Binary Cycle Diagram 
for this plant and working fluid (turbine exhaust occurs near the dome) and may be added in a future phase of development. Second, previous studies (1) have shown that although superheaters and regenerators improved the cycle thermodynamically, the improvement in cycle performance did not offset the accompanying cost increase for an experimental plant. 


\subsection{Assumptions Used for the Parametric Study of the Geothermal Binary Cycle}

The basic assumptions were as follows:

1. A11 pump efficiencies were assumed to be $80 \%$. Turbine efficiency was assumed to be $85 \%$ and electrical efficiency was assumed to be $95 \%$.

2. The limiting boiler pinch point was assumed to be $10^{\circ} \mathrm{F}$.

3. The overall pressure drop for each leg for the isobutane flow was set at 40 psid. Three psid was assumed across the isobutane condenser.

4. The total brine pump pressure rise was set equal to 480 psid due to system design requirements. This figure includes the pumping requirements for the down-hole pumps, the reinjection pumps (at 300 psia), line losses and elevation head requirements.

5. Cooling water system power consumption was assumed to be a 10 psid pressure drop in the condenser, the line pressure drops, a $40-\mathrm{ft}$ head rise required by the cooling tower, and tower fan power requirements.

6. The condenser water temperature inlet was set at $75^{\circ} \mathrm{F}$. This was obtained from cooling tower performance curves and the worst climatic conditions existing at Raft River. The water outlet of the condenser wás set at $95^{\circ} \mathrm{F}$. Allowing $10^{\circ} \mathrm{F}$ terminal difference for the heat exchanger, the isobutane condensing temperature was therefore set at $105^{\circ} \mathrm{F}$. Four degrees of subcooling were added to protect the isobutane circulating pump.

7. The overall heat-transfer coefficients (U'S) were derived from manufacturers data ${ }^{(2)}$ corresponding to a fouling factor of $0.003 \frac{\mathrm{hr}-\mathrm{ft}^{2}-{ }^{0} \mathrm{~F}}{\mathrm{Btu}}$. These are listed in Table I. 
TABLE I

Overall Heat Transfer Coefficients Used for Heat Exchanger Calculations

Heat Exchanger

Description

HP Boiler

HP Preheater

LP Boiler

LP. Preheater

Condenser
Fouling Factor $=0.003$

(Btu/hr- $-\mathrm{ft}^{2}-{ }^{\mathrm{O}} \mathrm{F}$ )

157

120

145

110

133 


\subsection{Medium Temperature Brine Cases}

\subsubsection{Single Boiler Cycles}

A plot of effectiveness vs boiler temperature for the single boiler system is found in Figure 2.2.1-1 for brine temperatures of $260^{\circ} \mathrm{F}, 280^{\circ} \mathrm{F}$, $290^{\circ} \mathrm{F}$, and $300^{\circ} \mathrm{F}$. For a given brine temperature, effectiveness increases as boiler temperature increases due to the increased turbine pressure ratio. The curve turns down for two reasons: As turbine inlet pressure increases, pumping requirements also increase, reducing usable power, and, as the boiler temperature approaches the brine temperature, the boiler pinch point limitations require increased brine flow, and effectiveness drops.

Figure 2.2.1-2 represents a cross-plot of effectiveness vs brine temperature for the most effective single boiler temperature at a given brine temperature. As brine temperature increases, effectiveness increases due to the higher temperature heat source. There is more energy available from the brine and therefore less brine flow is required. Since the boiler pinch point controls the brine flow rate as brine temperature increases, there is a larger log mean temperature difference for the preheater, allowing better heat transfer and a smaller preheater.

\subsubsection{Double Boiler Cycles}

Typical flow split variation curves for the $300^{\circ} \mathrm{F}, 290^{\circ} \mathrm{F}, 280^{\circ} \mathrm{F}$, and $260^{\circ} \mathrm{F}$ cases are shown in Figures 2.2.2-1 through 2.2.2-4. A complete set of curves can be found in Reference 3. Figure 2.2.2-1 shows the effect of varying the flow split for a brine inlet temperature equal to $280^{\circ} \mathrm{F}$, an HP boiler temperature of $240^{\circ} \mathrm{F}$, and an LP boiler temperature of $140^{\circ} \mathrm{F}$. This case is representative of those cycles in which the HP and LP boiler temperature difference and the HP boiler and brine inlet temperature difference are relatively large $\left(40^{\circ} \mathrm{F}\right)$. The graph actually shows two curves: The first, starting from a flow split of zero, is the effectiveness curve obtained while the HP boiler pinch 


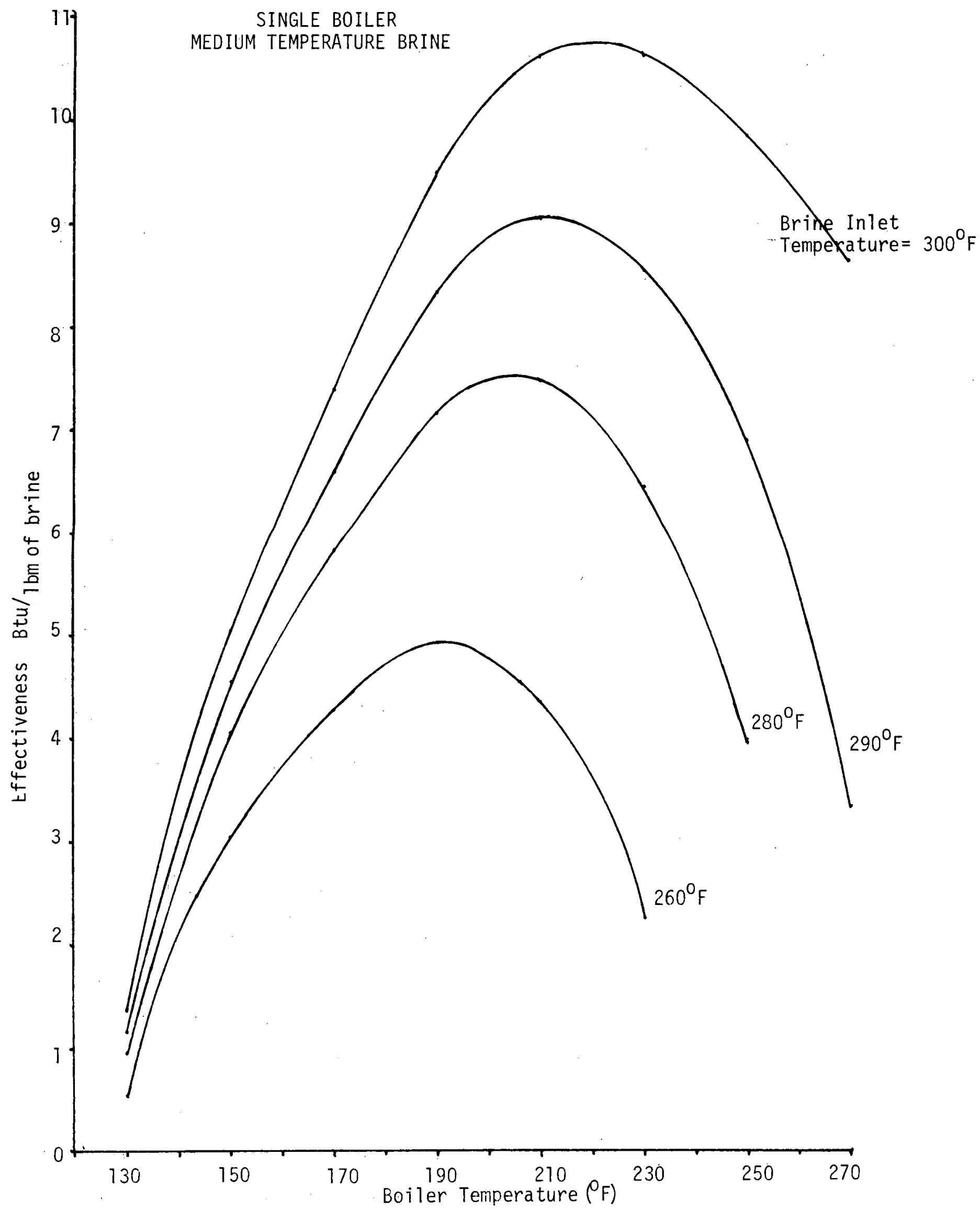

Figure 2.2.1-1 Effectiveness vs. Boiler. Temperature for Various Brine Temperatures, Single Boiler Case 


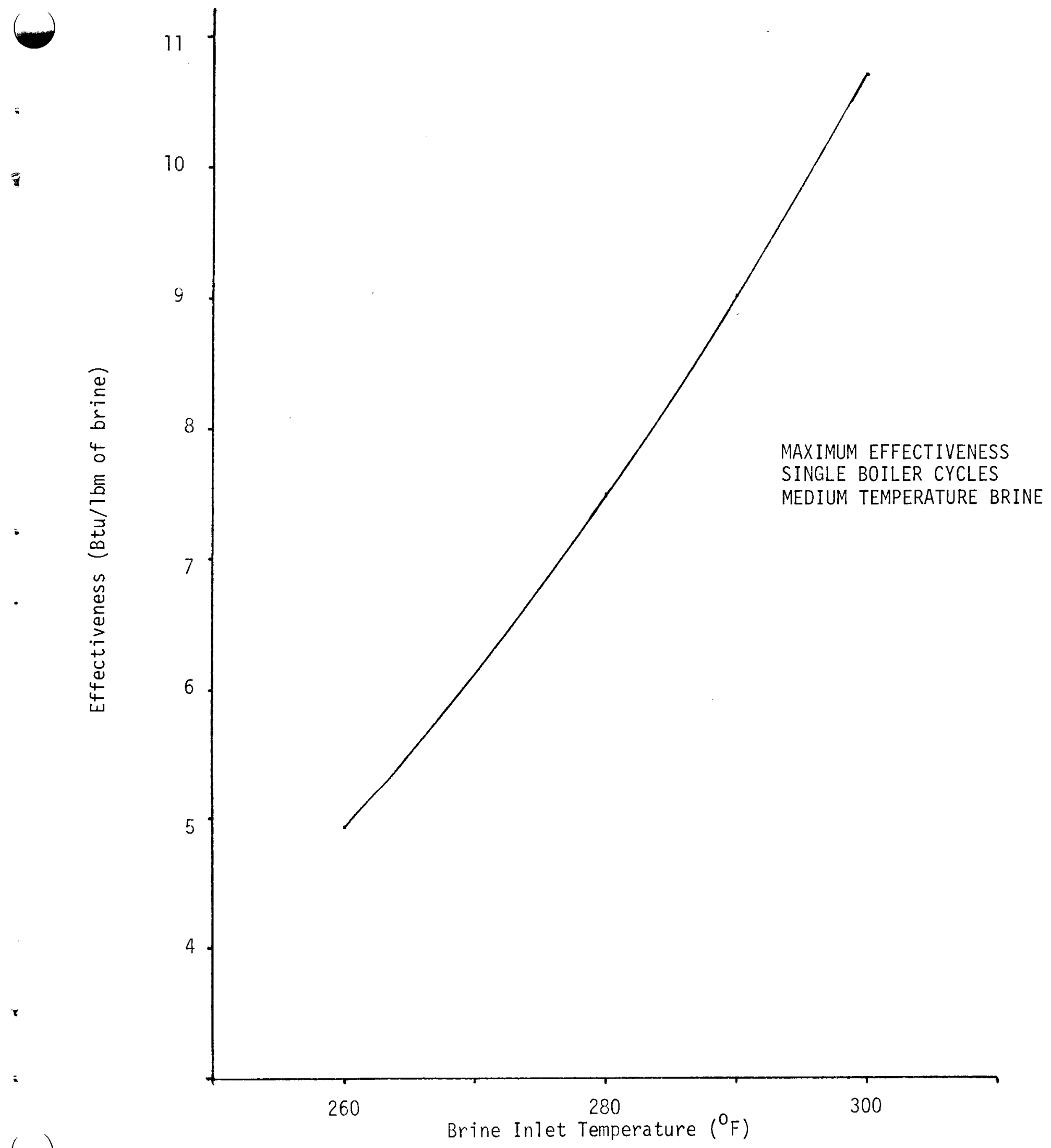

Figure 2.2.1-2 Maximum Effectiveness vs. Brine Inlet Temperature for Single Boiler Case 
point controls. The second curve, which intersects the first, is indicative of cycle performance when the flow split becomes large enough to shift control to the LP boiler pinch point. The intersection of the curves occurs at the point of maximum effectiveness, where both pinch points are equal to $10^{\circ} \mathrm{F}$. In almost all cases, it was found that the maximum effectiveness occurred at a flow split corresponding to both pinch points minimized at $10^{\circ} \mathrm{F}$. When the HP boiler pinch point controls, the brine temperature at the entrance to the HP preheater is fixed, thereby fixing the brine flow rate. The LP pinch point floats, depending upon the amount of brine energy remaining after the HP preheater and the LP boiler. As more isobutane flow is channeled to the LP flow path (flow split is increased), the LP pinch point decreases to the minimum value, and both pinch points are minimized. If the flow split is further increased, the control shifts to the LP boiler pinch point. The brine temperature at the entrance to the LP preheater is fixed and brine flow must increase to maintain that temperature as the LP boiler energy demands increase. Then, the HP pinch point increases due to the decrease in HP boiler requirements (less isobutane flow) and the increased brine flow rate.

There appears to be a point of higher effectiveness, as the curve bends backwards, with increasing effectiveness at a flow split of about 1.15. This is on the LP pinch point controlling curve, in the portion where the LP boiler pinch point is $10^{\circ} \mathrm{F}$, and the HP boiler pinch point as allowed to drop below $10^{\circ} \mathrm{F}$. Decreasing the pinch point allows more energy to be extracted from the brine, thereafter effectiveness increases. However, so does the heat exchanger area, and therefore plant cost. A pinch point of $0.0^{\circ} \mathrm{F}$, requiring infinite heat exchanger area, would result in the most effective cycle. Since the minimum allowable pinch point is $10^{\circ} \mathrm{F}$ for this study, the higher effectiveness point is not included. 
MEDIUM TEMPERATURE BRINE

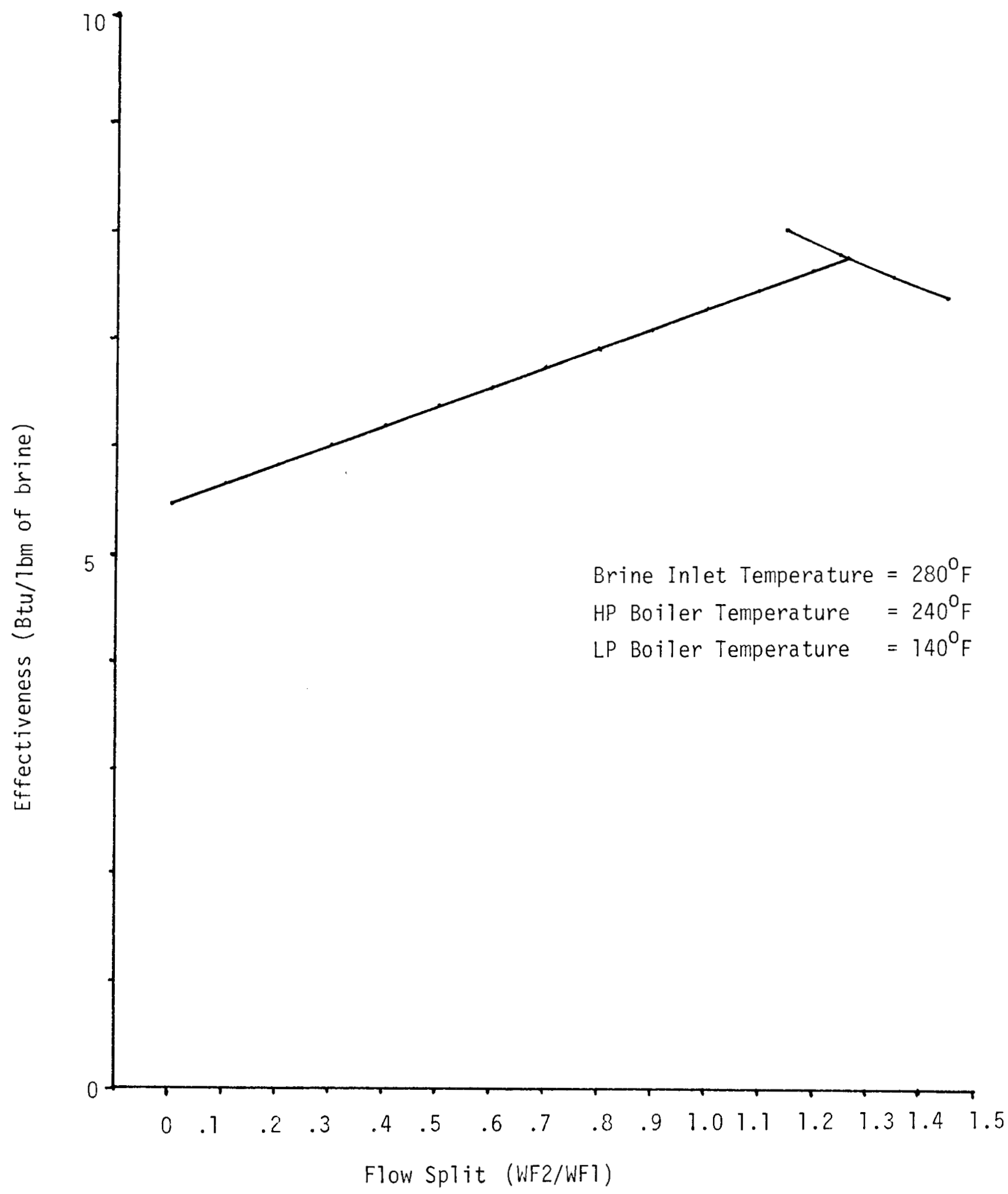

Figure 2.2.2-1 Sensitivity Study for Double Boiler Cycle - Flow Split vs. Effectiveness 


\section{MEDIUM TEMPERATURE BRINE}

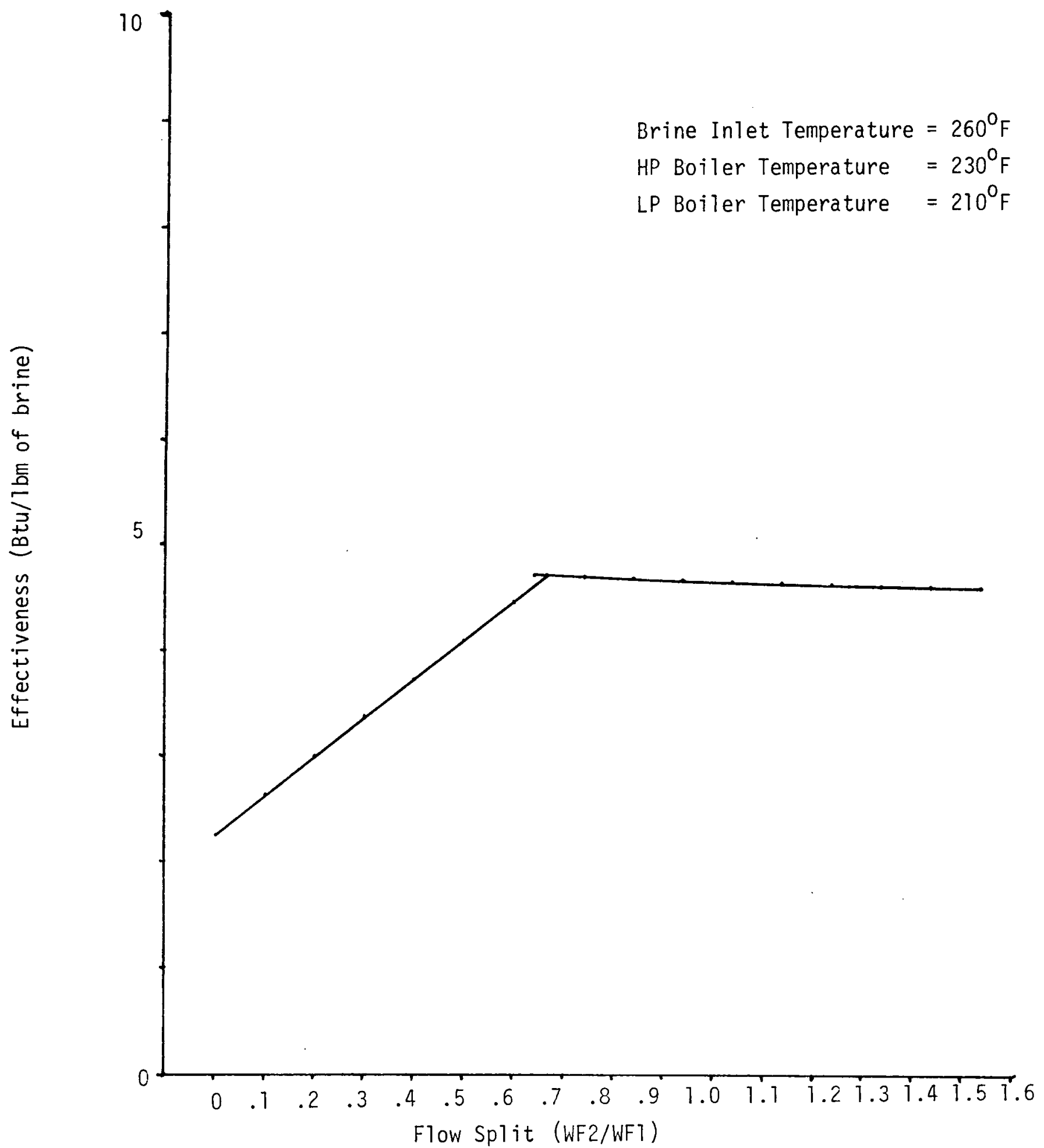

Figure 2.2.2-2 Sensitivity Study for Double Boiler Cvcle - Flow SDlit vs. Effectiveness 
Figure 2.2.2-2 illustrates a cycle in which the boiler temperatures are closer together and closer to the brine inlet temperature. For this case there is a brine inlet temperature of $260^{\circ} \mathrm{F}$, and $\mathrm{HP}$ boiler temperature of $230^{\circ} \mathrm{F}$ and the LP boiler temperature of $210^{\circ} \mathrm{F}$. The curves are similar to those in Figure 2.2.2-1, but the curve which indicates LP boiler pinch point control is flatter. This means the effectiveness is less sensitive to increases in flow split after the shift point (flow split $=.66)$. In other words, since the two boiler temperatures are relatively close together, the effect of shifting flow to the LP path is less pronounced than in Figure 2.2.2-1, where the LP boiler temperature is significantly lower than the HP boiler temperature.

This flattening effect is more pronounced in Figure 2.2.2-3 which shows the case of brine inlet temperature equal to $300^{\circ} \mathrm{F}$, with an HP boiler temperature of $230^{\circ} \mathrm{F}$, and an LP boiler temperature of $210^{\circ} \mathrm{F}$. In this instance there are two factors which contribute to producing a system which is quite insensitive to flow split increases: The brine temperature is significantly higher than the HP boiler temperature, and the two boiler temperatures are close together. The large temperature difference between the brine and the HP boiler causes the poinch point control shift to occur early (flow split <.1). The fact that the difference between the HP and LP boiler temperatures is small makes the curve flat, since the system sees little difference in specific power output or specific pumping power requirement between the two boilers. See Section 2.2.3 for a more complete discussion of this phenomenon.

Figure 2.2.2-4 is an illustration of the exception to the rule that effectiveness is maximized where both pinch points are minimized. In this case, the brine temperature is $280^{\circ} \mathrm{F}$, the HP boiler temperature is $260^{\circ} \mathrm{F}$, and the LP boiler temperature $\left(110^{\circ} \mathrm{F}\right)$ is very close to the condensing temperature $\left(105^{\circ} \mathrm{F}\right)$. This results in a pressure ratio of only 1.07 across the LP turbine, producing 


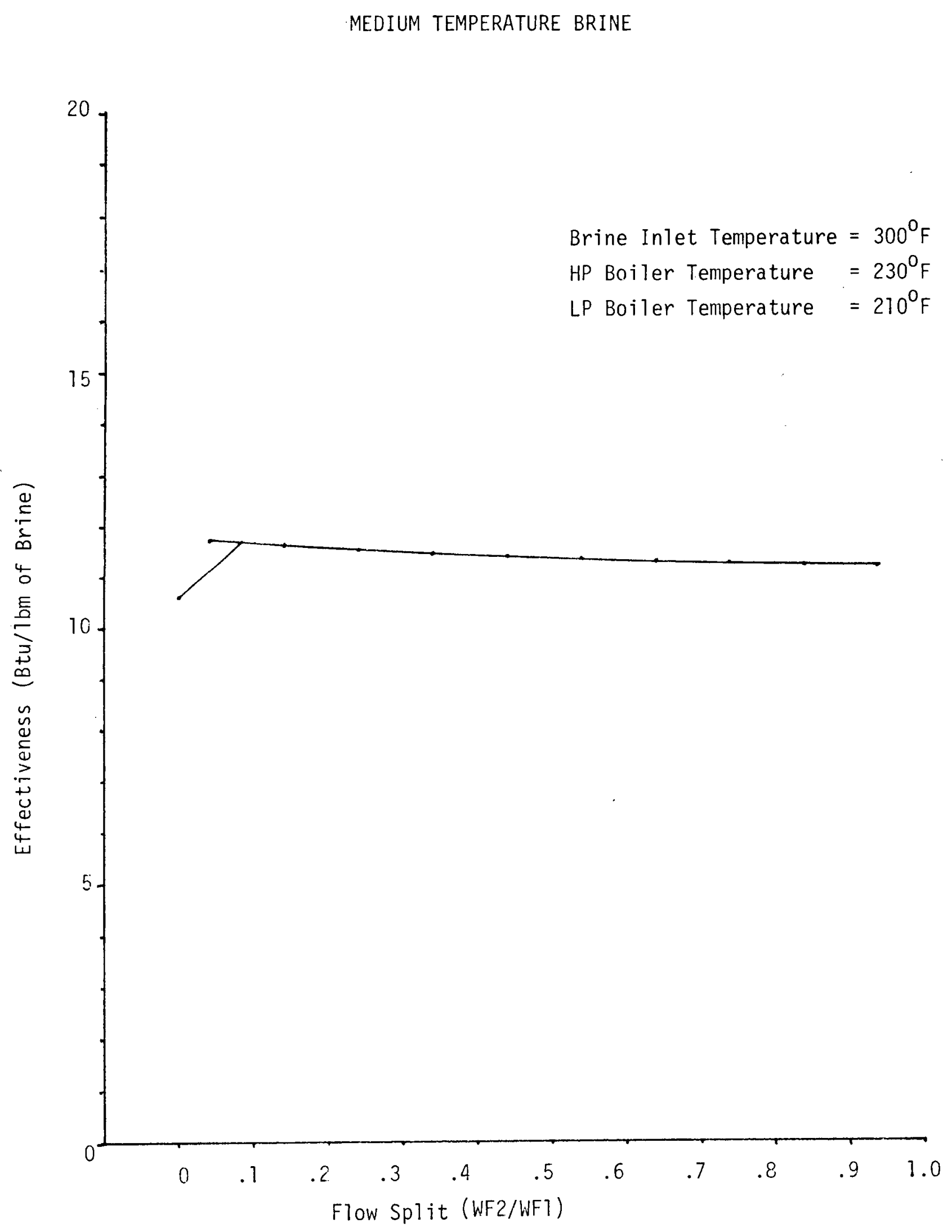

Figure 2.2.2-3 Sensitivity Study for Double Boiler Cvcle - Flow Split vs. Effectiveness 


\section{MEDIUM TEMPERATURE BRINE}

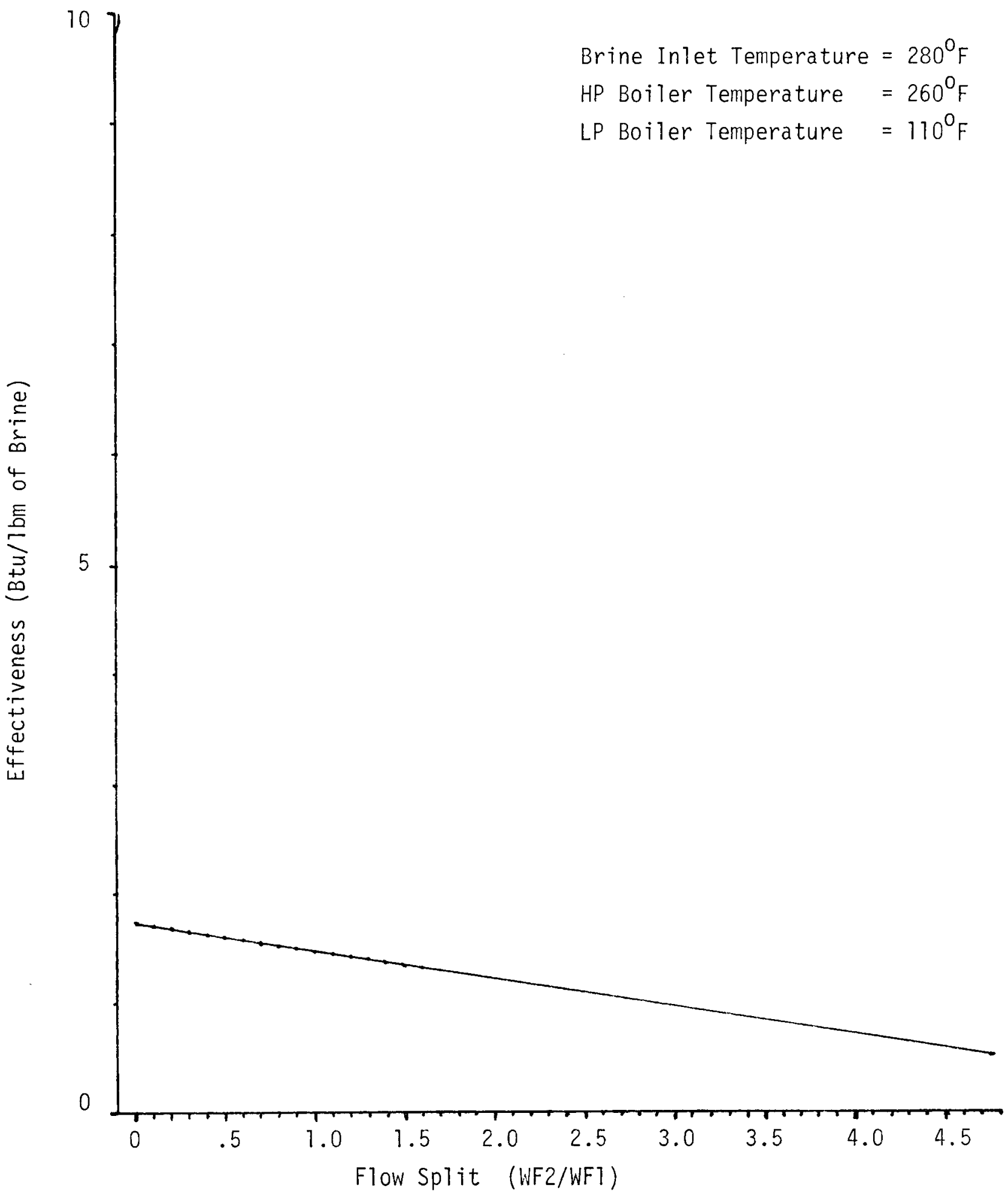

Figure 2.2.2-4 Sensitivity Study for Double Boiler Cvcle - Flow Snlit vs. Effectiveness 
very little power per pound of isobutane. In fact, the parasitic losses for the LP loop are greater than the turbine output, resulting in a negative net power out of the LP turbine. Therefore, any increase of flow to the LP loop would decrease effectiveness.

Figures 2.2.2-5 through 2.2.2-8 are plots of effectiveness vs LP boiler temperature for several HP boiler temperatures at brine temperatures of $260^{\circ} \mathrm{F}$, $280^{\circ} \mathrm{F}, 290^{\circ} \mathrm{F}$, and $300^{\circ} \mathrm{F}$. The points on these curves represent the most effective case for each pair of boiler temperatures, as found from the flow split curves. At the maximum effectiveness, both boiler pinch points are minimized at $10^{\circ} \mathrm{F}$. Figures 2.2.2-9 through 2.2.2-11 are cross plots of the above curves, showing effectiveness vs HP boiler temperatures. The $260^{\circ} \mathrm{F}$ brine cases were not cross plotted because only two LP boiler temperatures were studied.

Figure 2.2.2-12 is a plot of effectiveness vs brine temperature. The effectiveness is that for the most effective boiler combination for each brine temperature. As in the single boiler case, the effectiveness increases with brine temperature.

\subsubsection{Results of the Medium Temperature Brine Cases}

All of the flow split plots for the double boiler cases include a point of flow split equals zero, or the single boiler case at the HP boiler temperature. With the exception noted in Section 2.2.2, at a given brine temperature, the maximum effectiveness for each two-boiler combination was found at the flow split were both pinch points were minimized at $10^{\circ} \mathrm{F}$, and the single boiler cases were less effective than any two-boiler temperature. The boiler pinch point controls the brine temperature at the boiler exit, and the brine is rejected from the system at elevated temperatures. The LP boiler and preheater utilize the previously wasted energy, and the cycle becomes more effective. A 


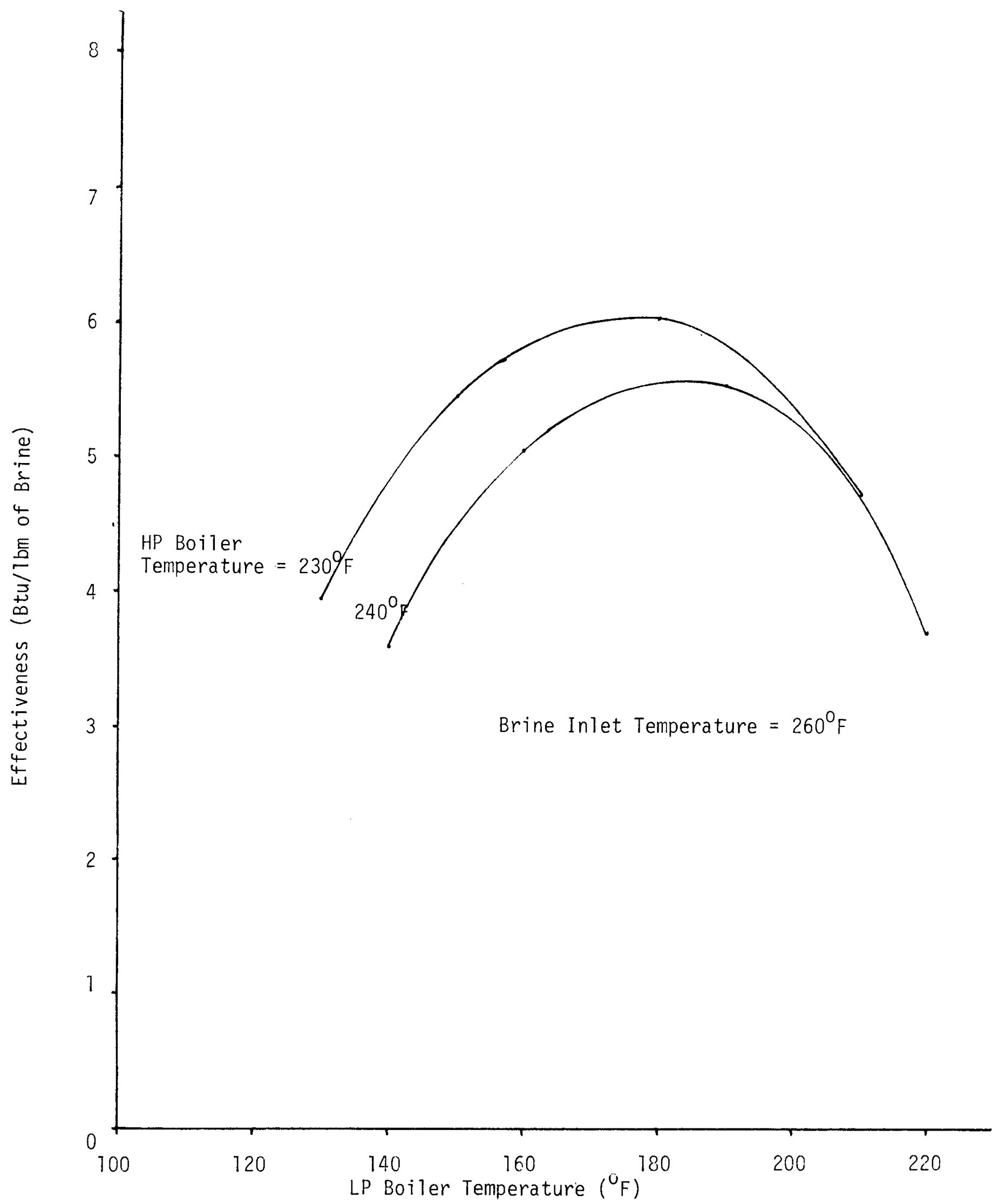

Figure 2.2.2-5 Effectiveness VS LP Boiler Temperature for Varying HP Boiler Temiperatures 


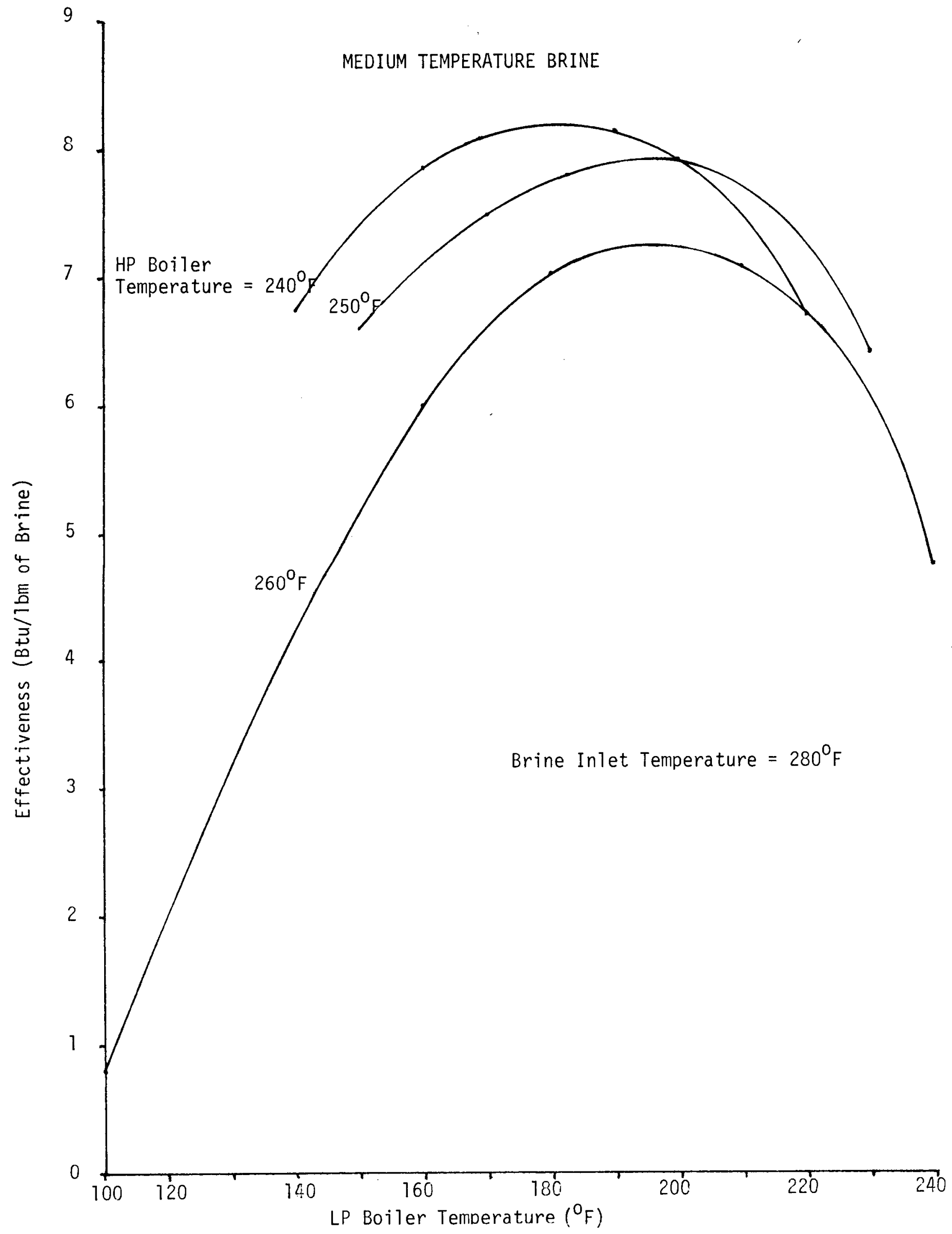
Figure 2.2.2-6 $\begin{aligned} & \text { Effectiveness vs LP Boiler Temperature for Varying HP Boiler } \\ & \text { Teiliperatures }\end{aligned}$ 
MEDIUM TEMPERATURE BRINE

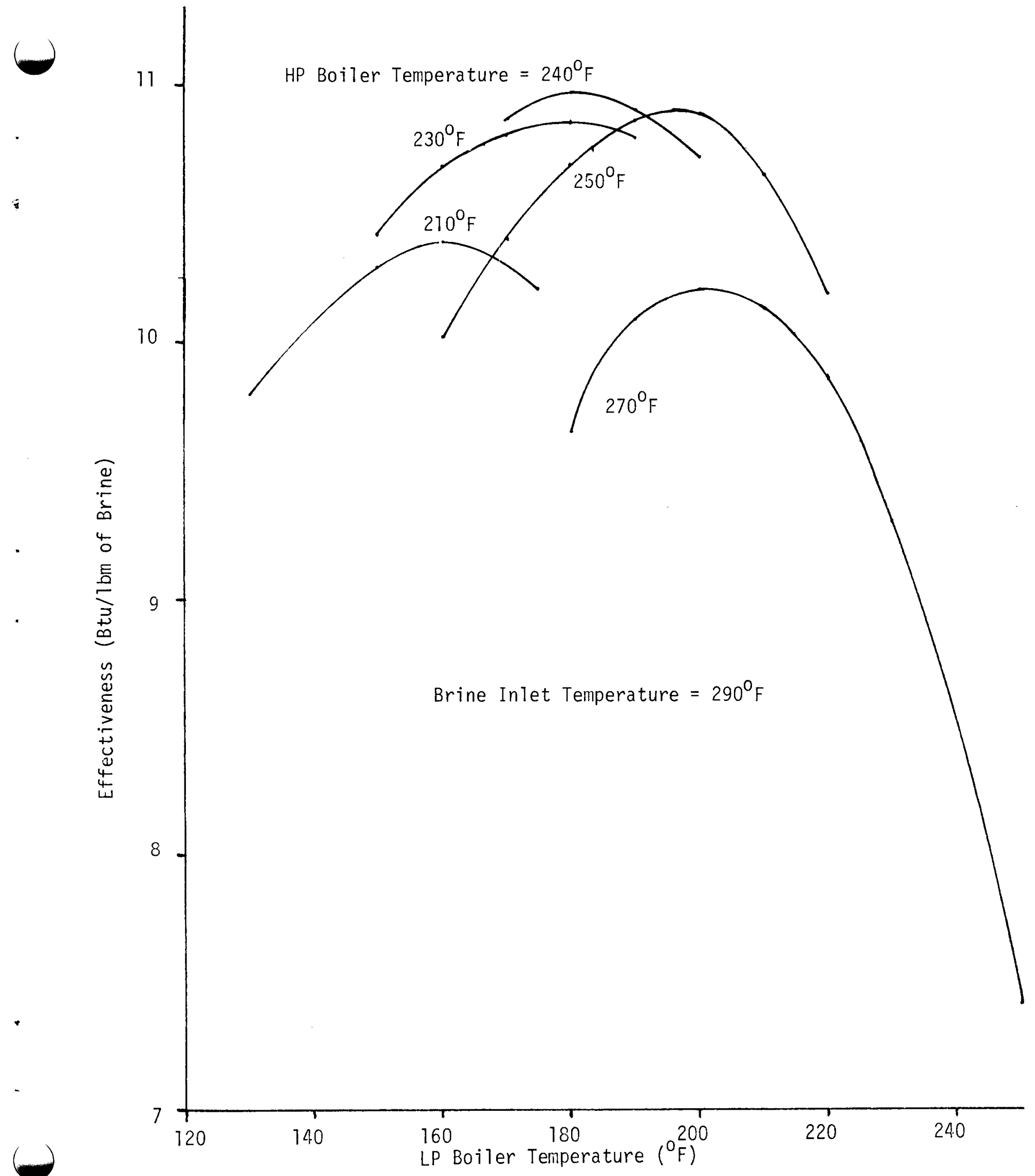

Figure 2.2.2-7 Effectiveness vs LP boiler Temperature for Varying HP Boiler Temperatures 


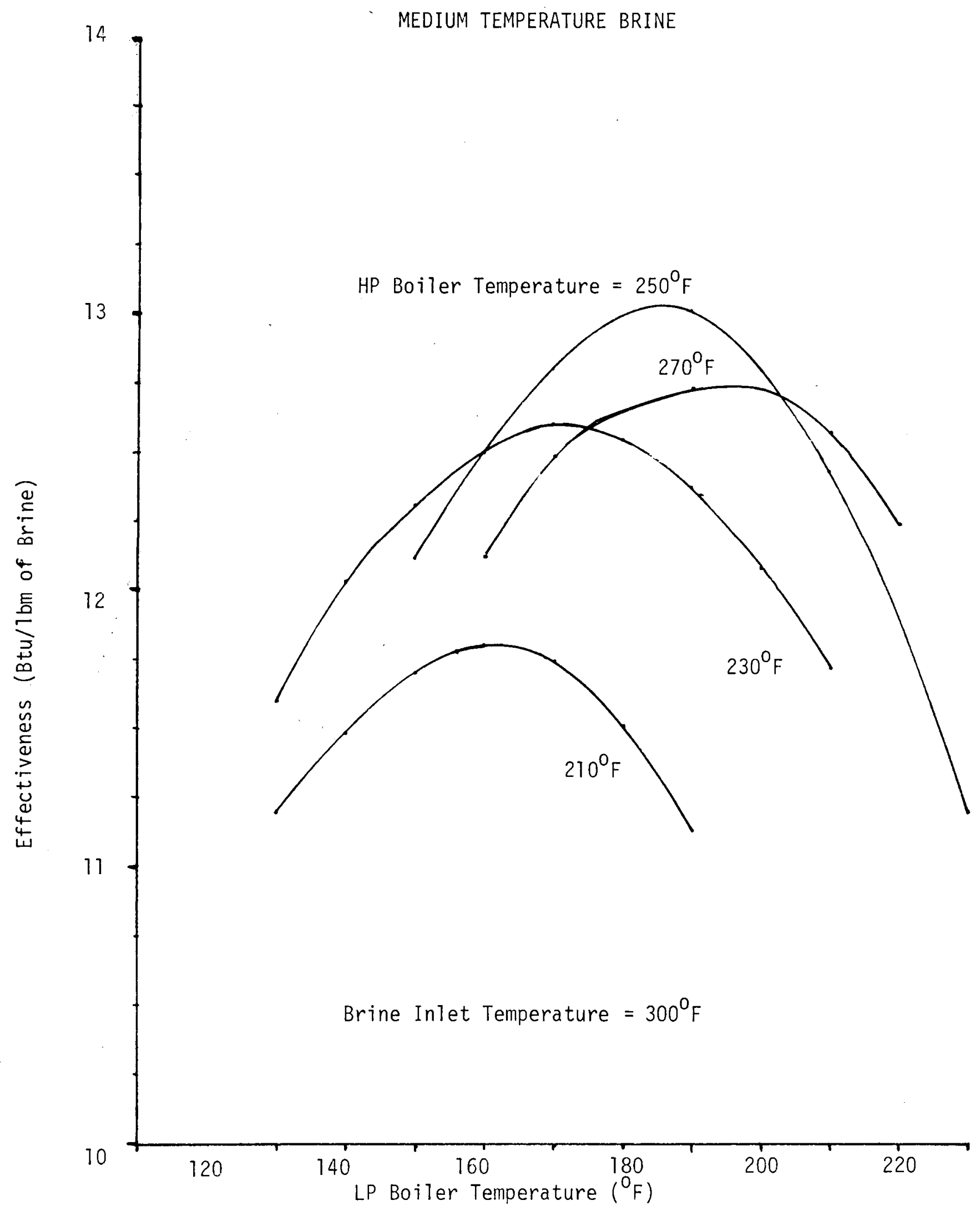

Figure 2.2.2-8 Effectiveness vs LP boiler Temperature for Varying HP Boiler Temperatures 


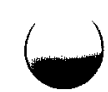

$\div$

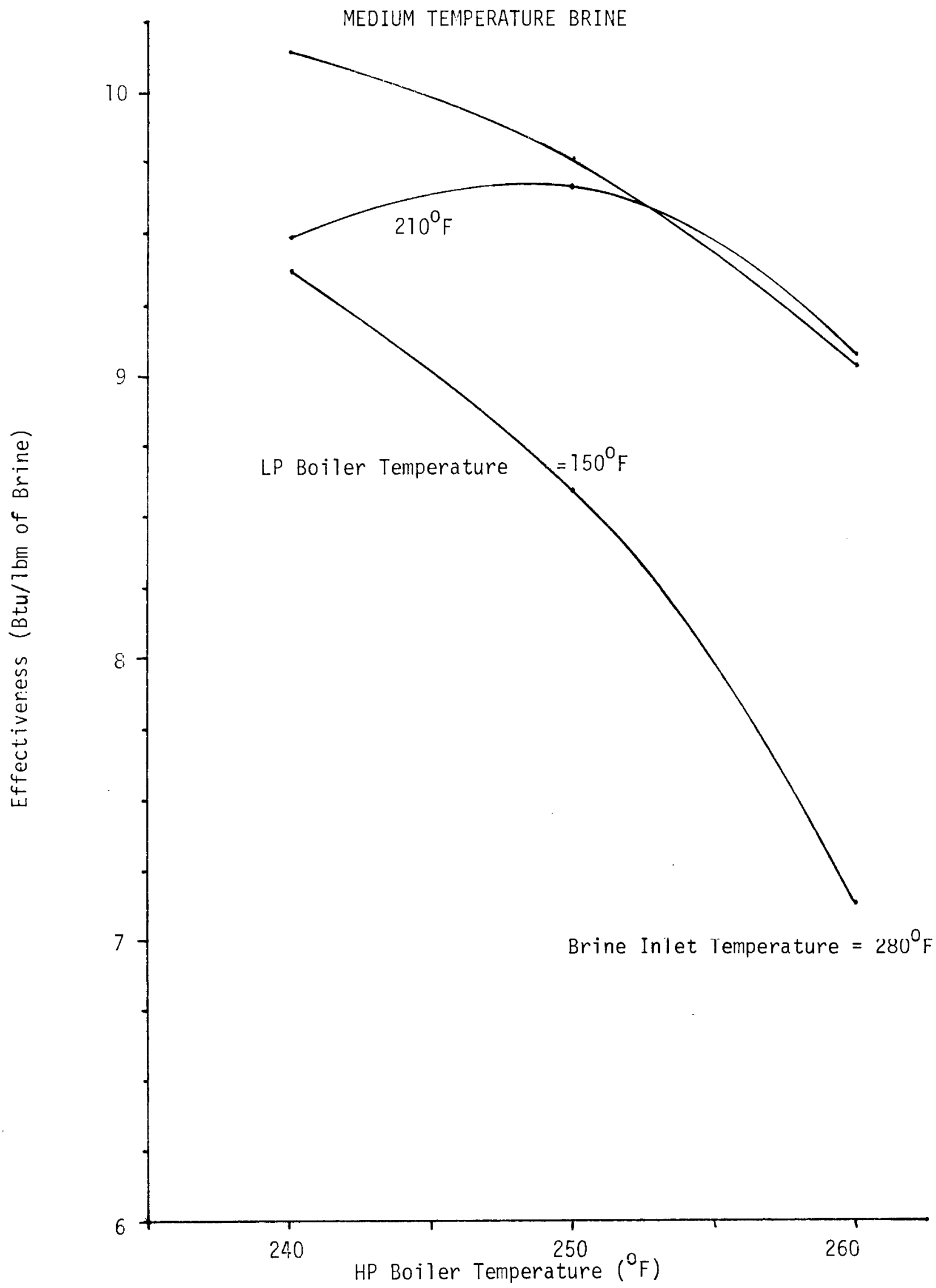

Figure 2.2.2-9 Effectiveriess vS HP Boiler Temperature for Varying LP Boiler Temperatures 


\section{MEDIUM TEMPERATURE BRINE}

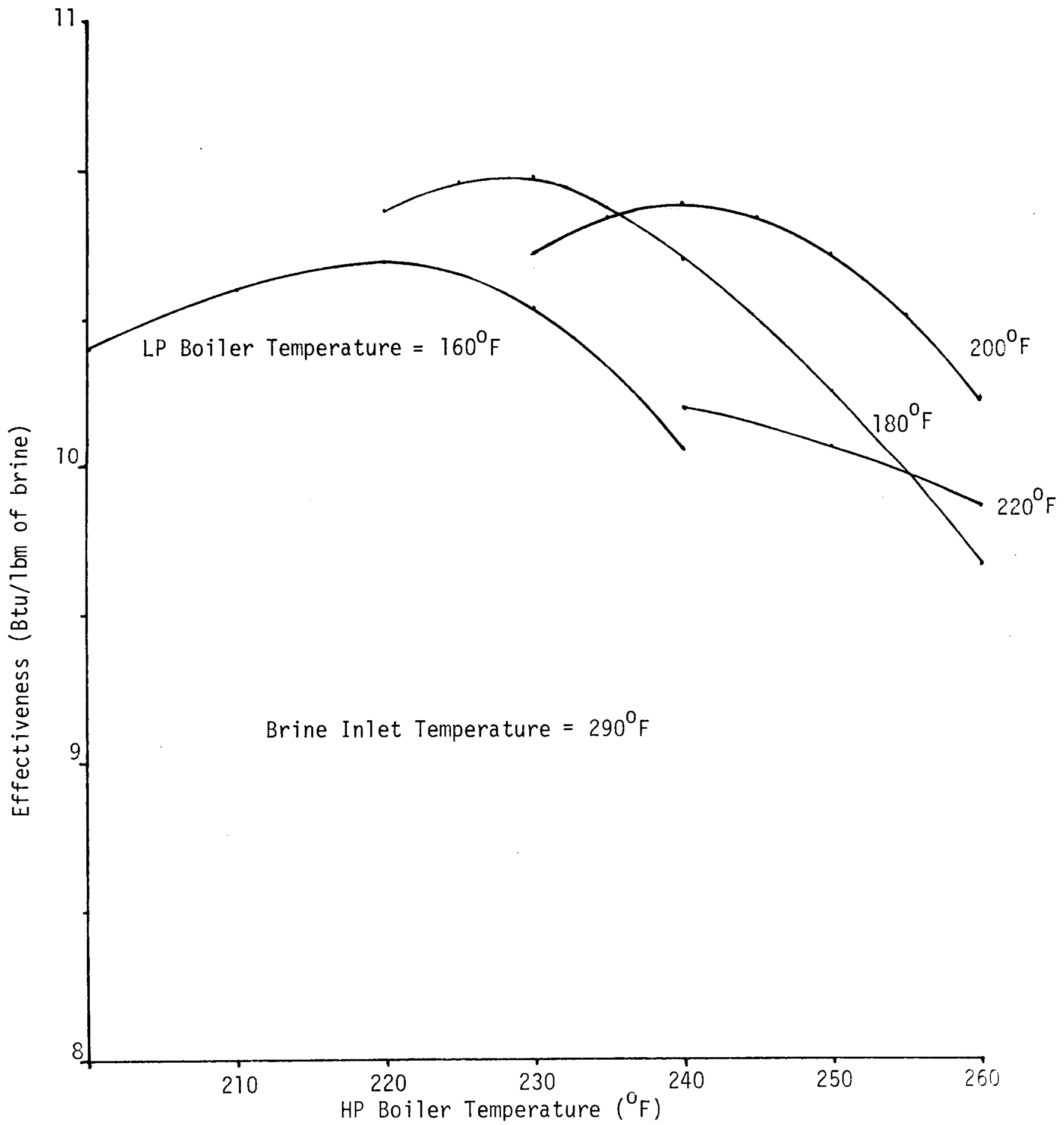

Figure 2.2.2-10 Effectiveness vs HP Boiler Temperature for Varying LP Boiler Temperatures 


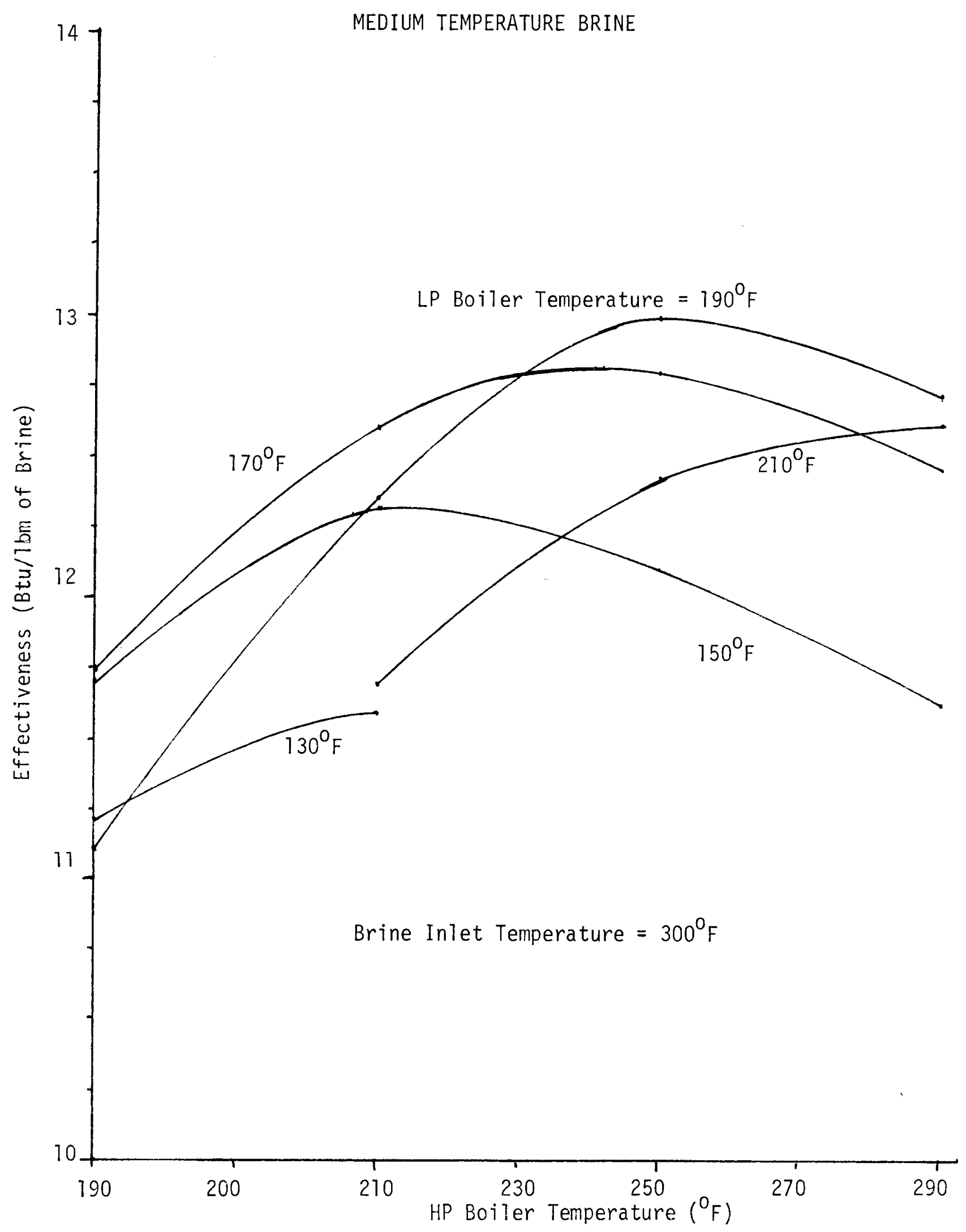

Figure 2.2.2-11 Effectiveness vS HP Boiler Temperature for Varvina LP Boiler Temperatures 


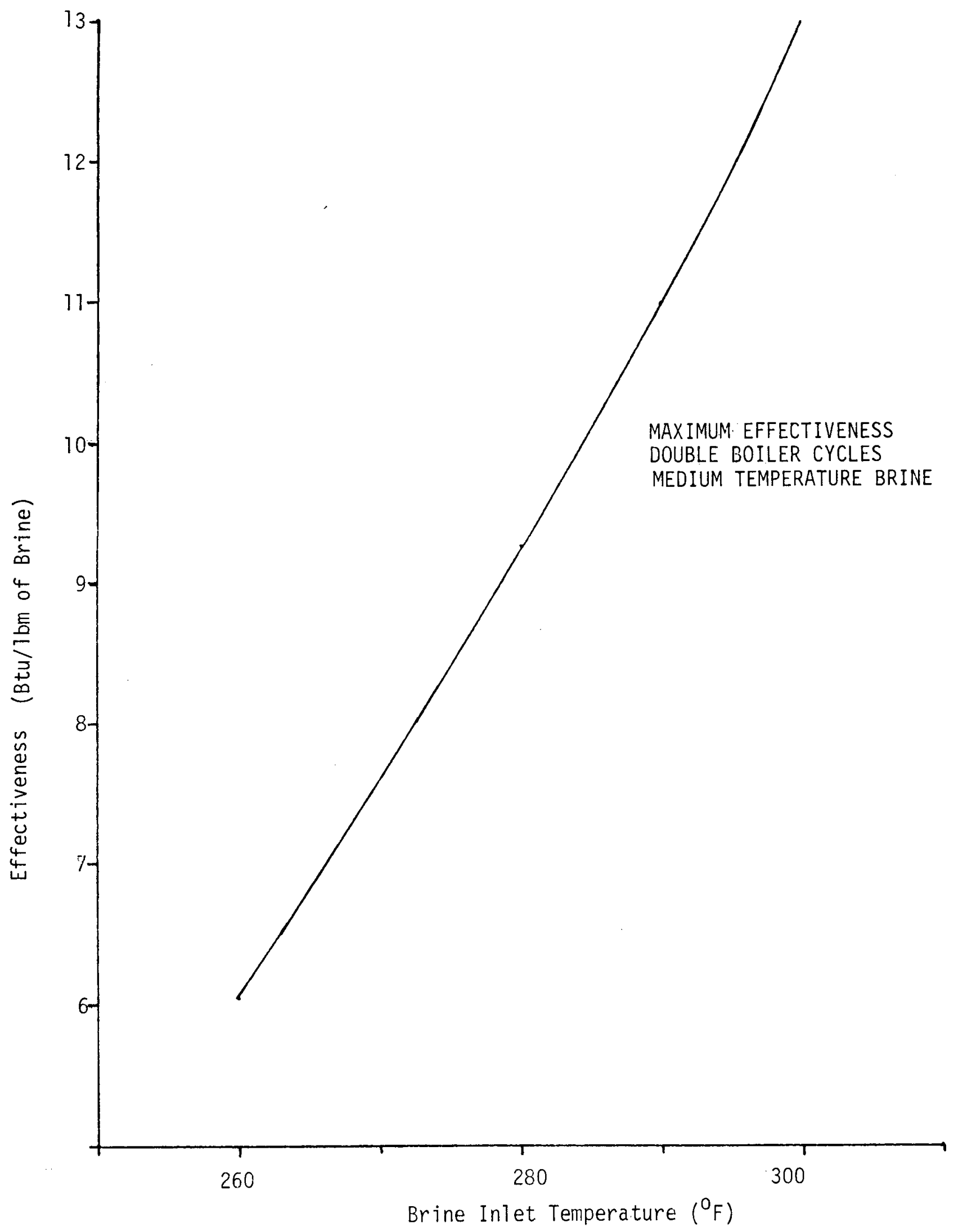

Figure 2.2.2-12. Maximum Effectiveness vs. Brine Inlet Temperature Double Boiler Case 
third boiler would further increase the cycle effectiveness, but the increase is marginal compared to the cost of heat exchangers, pumps, turbines, etc. For purposes of comparison, the maximum effectivenesses obtainable for a brine temperature are plotted in Figure 2.2.3-1 for the single and double boiler cases. This is a combination of Figure 2.2.1-2 and Figure 2.2.2-12. The double boiler combinations run between $20-25 \%$ more effective than the single boiler cases for a given brine temperature.

The point of maximum effectiveness, where both pinch points are mininized, occurs at the flow split at which pinch point control shifts from the HP boiler pinch point to the LP boiler pinch point. This "shift point" is dependent upon the relationship between the brine inlet temperature (TB1), HP boiler temperature (TF1), and LP boiler temperature (TF2). Figure 2.2.3-2 i11ustrates the "shift point" dependency upon boiler temperatures for a given brine inlet temperature of $300^{\circ} \mathrm{F}$. As TBT-TFl increases, the "shift point" moves toward a flow split of zero, or the single boiler case. But for a given TB1-TFl, as TFITF2 increases, the "shift point" also increases. Therefore, for a set TB1, the "shift point" approaches zero as TF1-TF2 approaches zero and as TBT-TF1 gets large.

The "shift point" also appears to be dependent upon the value of brine inlet temperature. Figure 2.2.3-3 shows the relationship between brine temperature and shift point for two values of TBl-TFI, $20^{\circ} \mathrm{F}$ and $30^{\circ} \mathrm{F}$. As previously noted, the "shift point" decreases as TF1-TF2 decreases. A1so, as TB1 increases the "shift point" decreases. Therefore, for a set TB1-TFl, the "shift point" approaches zero as TF1-TF2 approaches zero and as TB1 gets 1arge.

Combining these trends, then, as the brine temperature increases, and as TB1-TF1 increases, and as TF1-TF2 decreases, the "shift point" approaches 


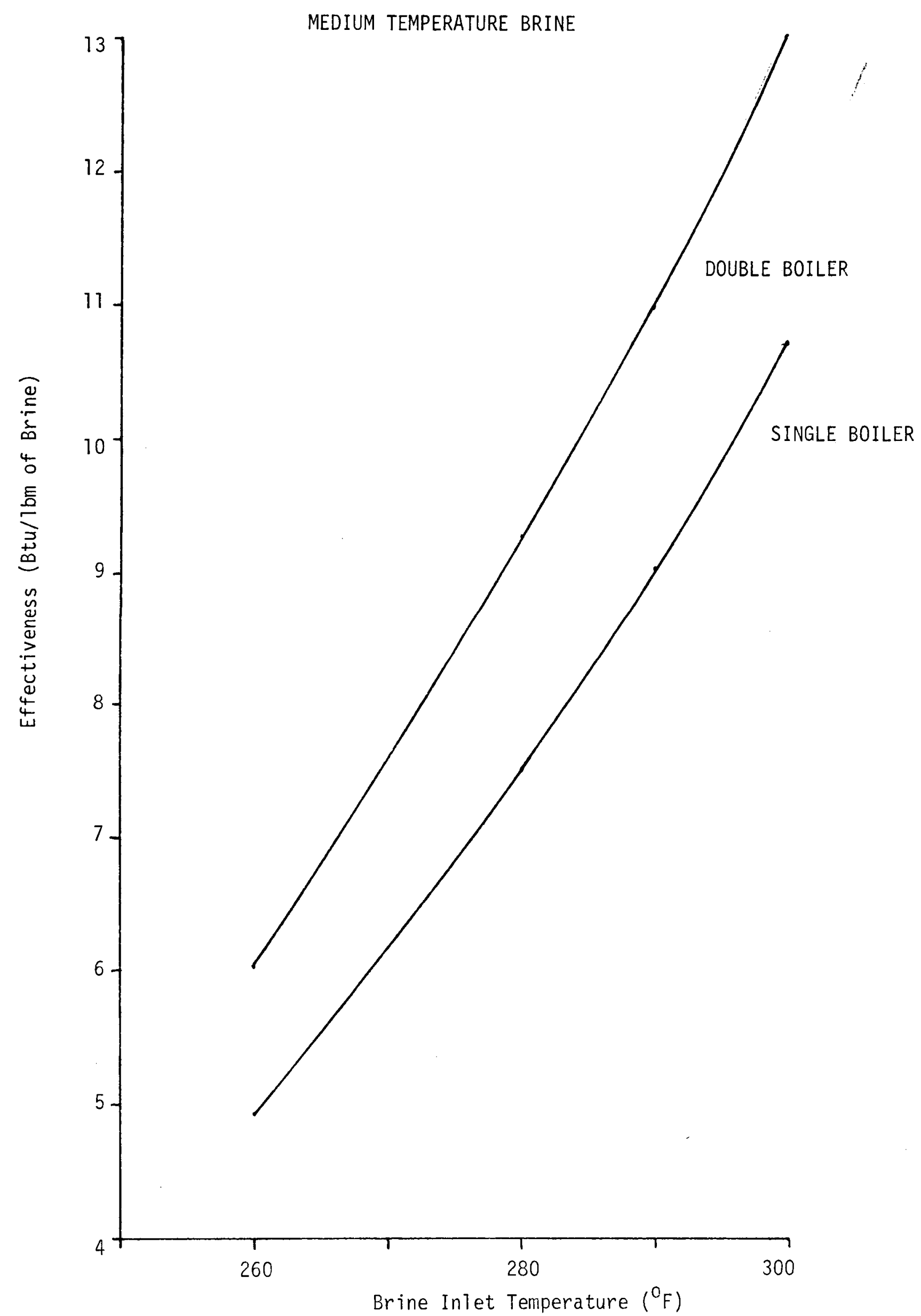

Figure 2.2.3-1 Comparison of Maximum Effectiveness vs Brine Inlet Temperature for Single and Double Boiler Cases 
zero, or the single boiler case. At the "shift point" neither pinch point controls, both pinch points are minimized and effectiveness is maximized.

Physically then, the "shift point" approaches zero when the brine temperature is relatively high, the LP boiler temperature is comparatively low, and the LP boiler temperature is very close to the HP boiler temperature. 


\section{MEDIUM TEMPERATURE BRINE}

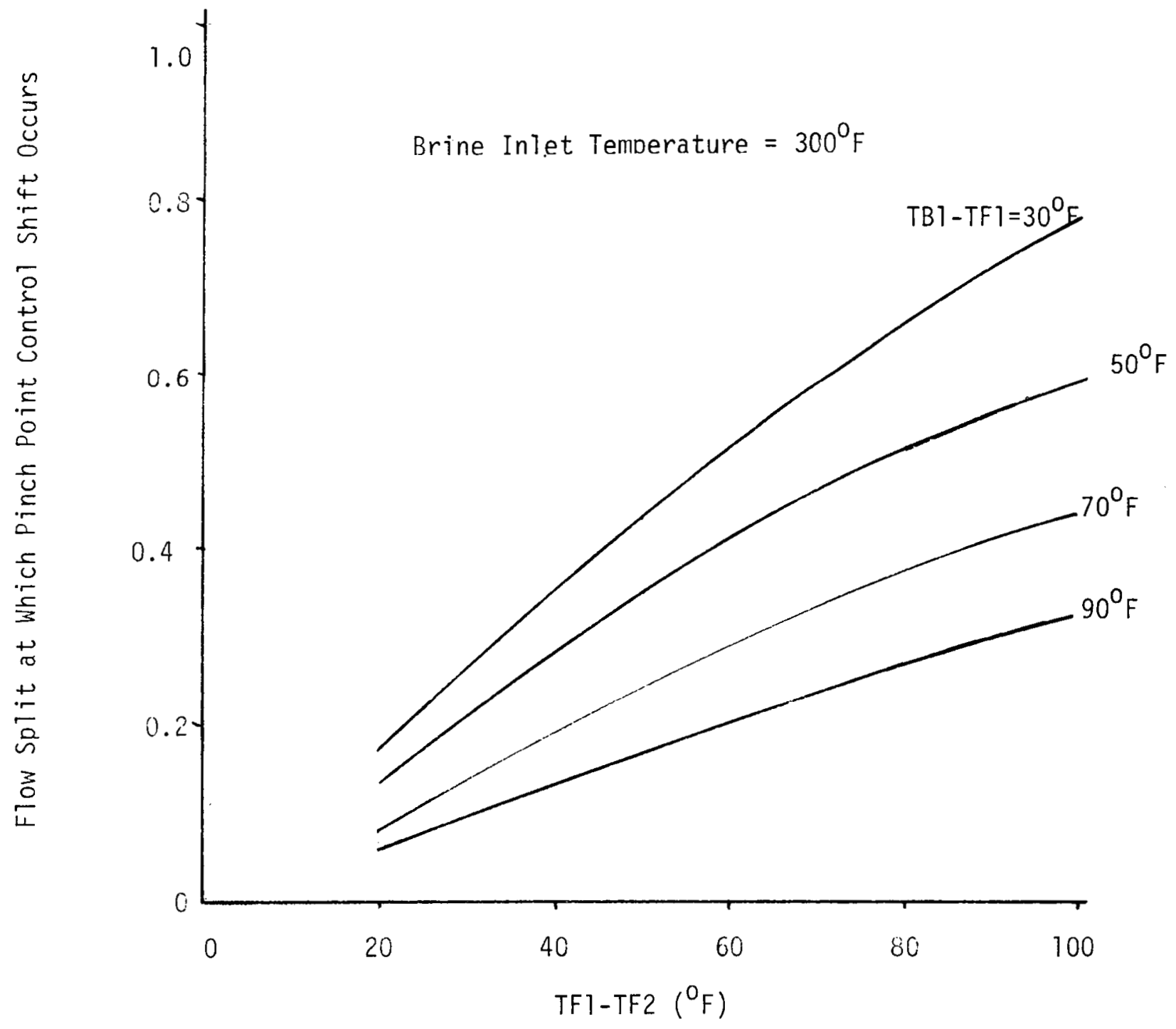

Figure 2.2.3-2 Flow Split at which pinch point control shift occurs vs the temperature difference between the HP and LP boiler for several temperature differences between the brine and $\mathrm{Hp}$ boiler. Brine inlet temperature $=300^{\circ} \mathrm{F}$ 


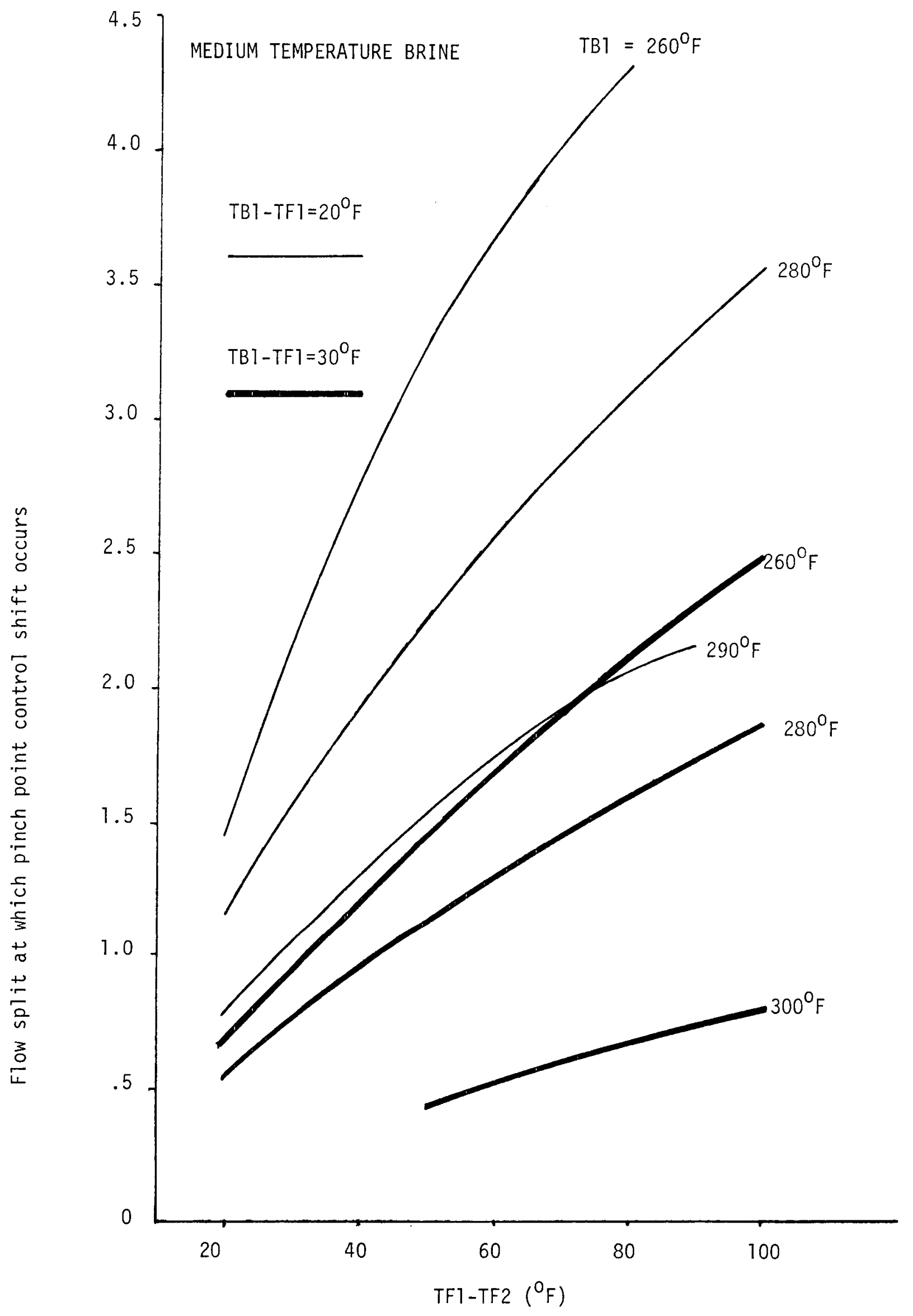

Figure 2.2.3-3 Flow Split at which pinch point control shift occurs vs the temperature difference between the HP and LP boilers for various temperature differences between the brine and HP boiler 


\subsection{High Temperature Brine Cases}

\subsubsection{Single Boiler Cycles}

Single boiler cases were run for brine temperatures of 320,340 , and $360^{\circ} \mathrm{F}$.

Figure 2.3.1-1 is a plot of effectiveness vs boiler temperature for those brine temperatures. As expected, effectiveness increases with boiler temperature due to the increased turbine pressure ratio. The effectiveness values themselves are high compared to the medium temperature brine cases. This effect is caused by the fact that less high temperature brine flow is required to supply the same amount of energy at a given medium temperature flow rate.

The effectiveness curve of the $320^{\circ} \mathrm{F}$ brine case makes a sharp bend at a boiler temperature of about $250^{\circ} \mathrm{F}$ and continues to rise instead of laying over like the $340^{\circ} \mathrm{F}$ and $360^{\circ} \mathrm{F}$ brine cases. The reason for this is found in the relationship between boiler pinch point, boiler latent heat of vaporization, and preheater energy requirements. Several things happen simultaneously as boiler temperature increases:

1. Isobutane flow decreases, because of the increased turbine output. This decreases the brine flow requirement.

2. The boiler pinch point fixes the brine temperature at the boiler exit.

3. The preheater requirement increases because the isobutane must be heated to a higher temperature.

4. The latent heat of vaporization decreases. This means that brine flow further decreases due to the smaller energy requirement. In fact, the rate of decrease of the latent heat is very rapid near the top of the dome. It is down $30 \%$ from $260^{\circ} \mathrm{F}$ to $270^{\circ} \mathrm{F}$ as opposed to 1 ess than $10 \%$ per $10^{\circ} \mathrm{F}$ for boiler temperatures up to $250^{\circ} \mathrm{F}$.

5. It should te noted that at boiler temperatures of $250^{\circ} \mathrm{F}, 260^{\circ} \mathrm{F}$ and $270^{\circ} \mathrm{F}$, the turbine expands through the dome but exhausts into the superheat region. However, the maximum percentage liquid is less than $3 \%$ by volume, not a significant amount. Any degredation in turbine efficiency would be negligible, therefore no superheat was added to clear the dome. 
For the $320^{\circ} \mathrm{F}$ brine case, the decrease in latent heat is so rapid from $250^{\circ} \mathrm{F}$ to $270^{\circ} \mathrm{F}$ that the effectiveness climbs dramatically. The brine flow is great enough, however, that the brine energy remaining after the boiler demands is sufficient for preheating.

For the $340^{\circ} \mathrm{F}$ and $360^{\circ} \mathrm{F}$ brine cases, the latent heat at high broiler temperatures is small enough, and the brine temperature is high enough, that the brine flow is too low to provide energy for the increased preheater load when the boiler pinch point is fixed at its minimum, $10^{\circ} \mathrm{F}$. Therefore, the pinch point must be raised and brine flow must increase to accomplish the preheating. In fact, it is the preheater terminal difference which controls the brine flow rate instead of the boiler pinch point, and, because of the elevated pinch point, the $340^{\circ} \mathrm{F}$ and $360^{\circ} \mathrm{F}$ brine effectiveness curves lay over at high boiler temperatures. 


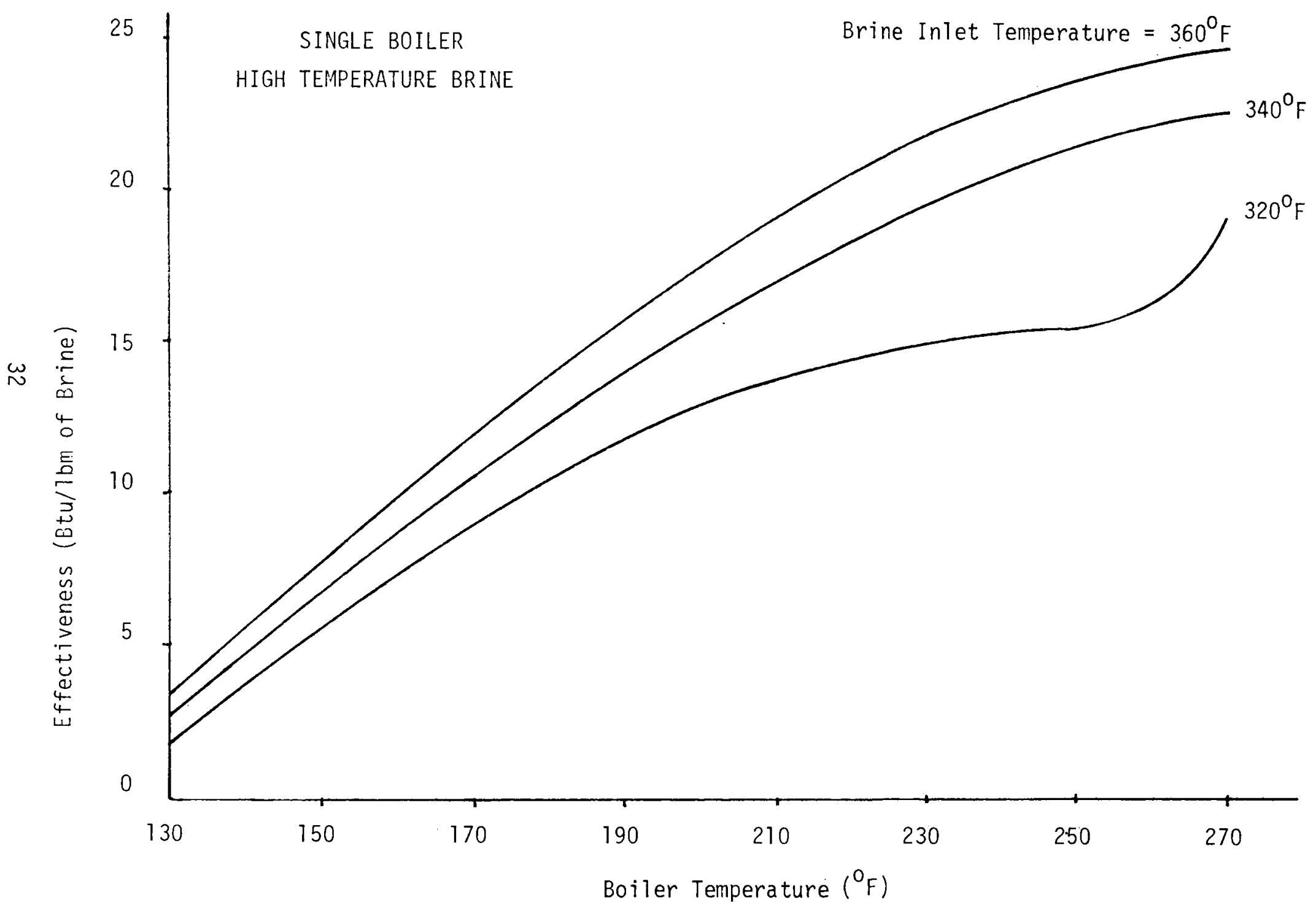

Figure 2.3.1-1 Boiler Temperature vs Brine Inlet Temperature for High Temperature Brine, Single Boiler 


\subsubsection{Supercritical Cycles}

For the supercritical cases, there is no discrete preheater or boiler, but a single heater. Since there is no boiler (no latent heat of vaporization), there is no pinch point defined. Several checks are made to ensure that there is no temperature overlap in the heater, but the pinch point limitations inherent in the boiler systems do not exist. The disadvantages of the supercritical cycles are the high operating pressures (critical pressure is 529 psia) which significantly increase the isobutane pumping power requirements.

Figures 2.3.2-1 through 2.2.2-3 show effectiveness vs turbine inlet temperature for brine temperatures of $360^{\circ} \mathrm{F}, 340^{\circ} \mathrm{F}$, and $320^{\circ} \mathrm{F}$. The turbine inlet pressures and temperatures were chosen such that an isentropic expansion would exhaust at the saturation line. The $360^{\circ} \mathrm{F}$ and $340^{\circ} \mathrm{F}$ brine cases exhibit increasing effectiveness with turbine inlet temperature until a maximum point is reached and the curve turns downward. This behavior is due to the fact that pressure is not a linear function of temperature, and pressure increases faster than temperature. The temperature increase causes a rise in thermodynamic efficiency. The pressure increase causes an increased pressure ratio across the turbine, and a larger gross power output. However, the pressure increase also builds up the pumping 1osses. At a trade-off point (maximum effectiveness) the pumping losses overshadow the thermodynamic efficiency increase, and the effectiveness drops.

Figure 2.3.2-3, the $320^{\circ} \mathrm{F}$ brine case, does not show a maximum effectiveness point at this lower brine temperature, the effect of raising turbine inlet temperature is less pronounced, and only the effect of the pumping power increase is seen. 


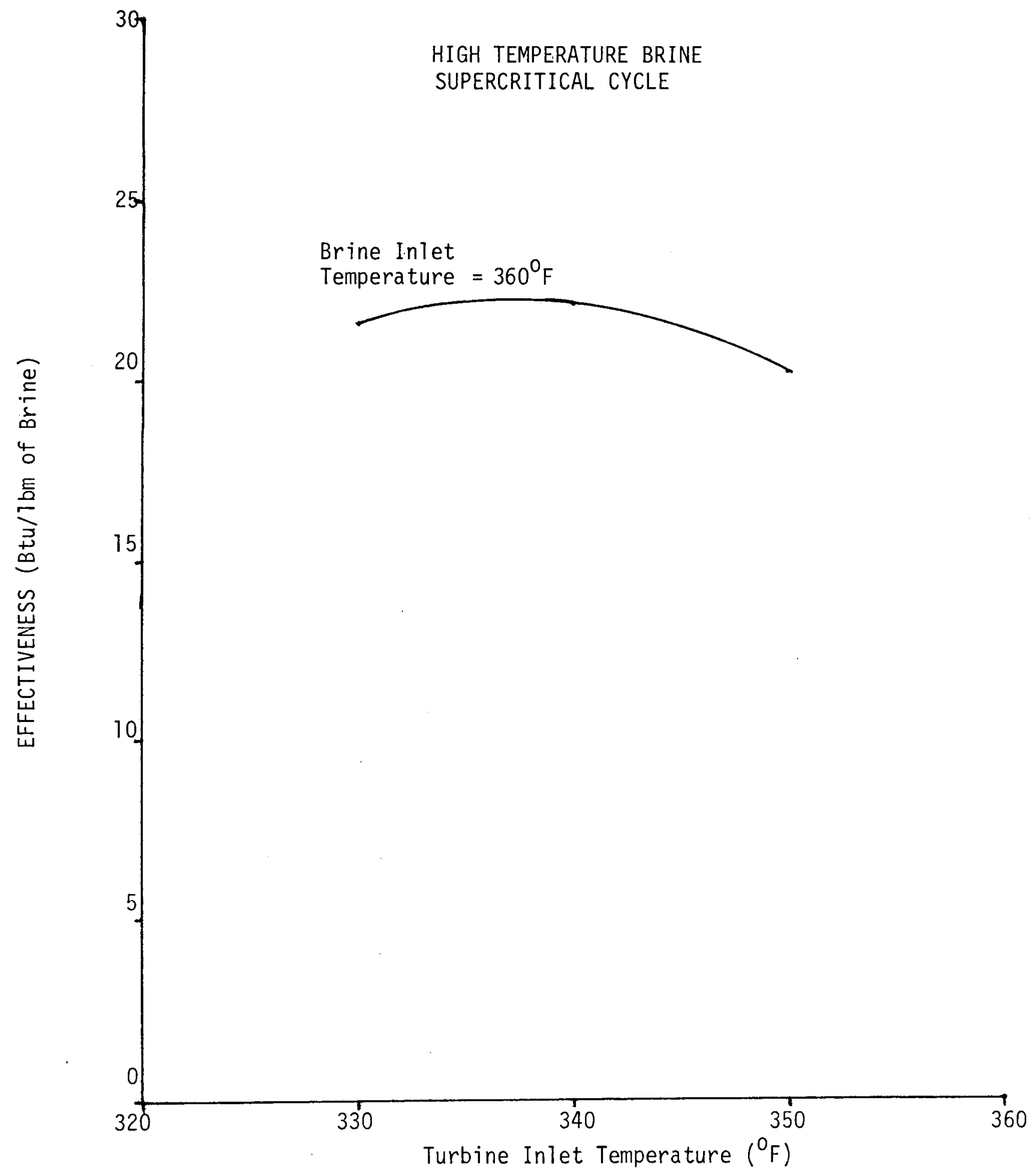

Figure 2.3.2-1 Turbine Inlet Temperature vs Effectiveness for the Supercritical Case $T B T=360^{\circ} \mathrm{F}$ 


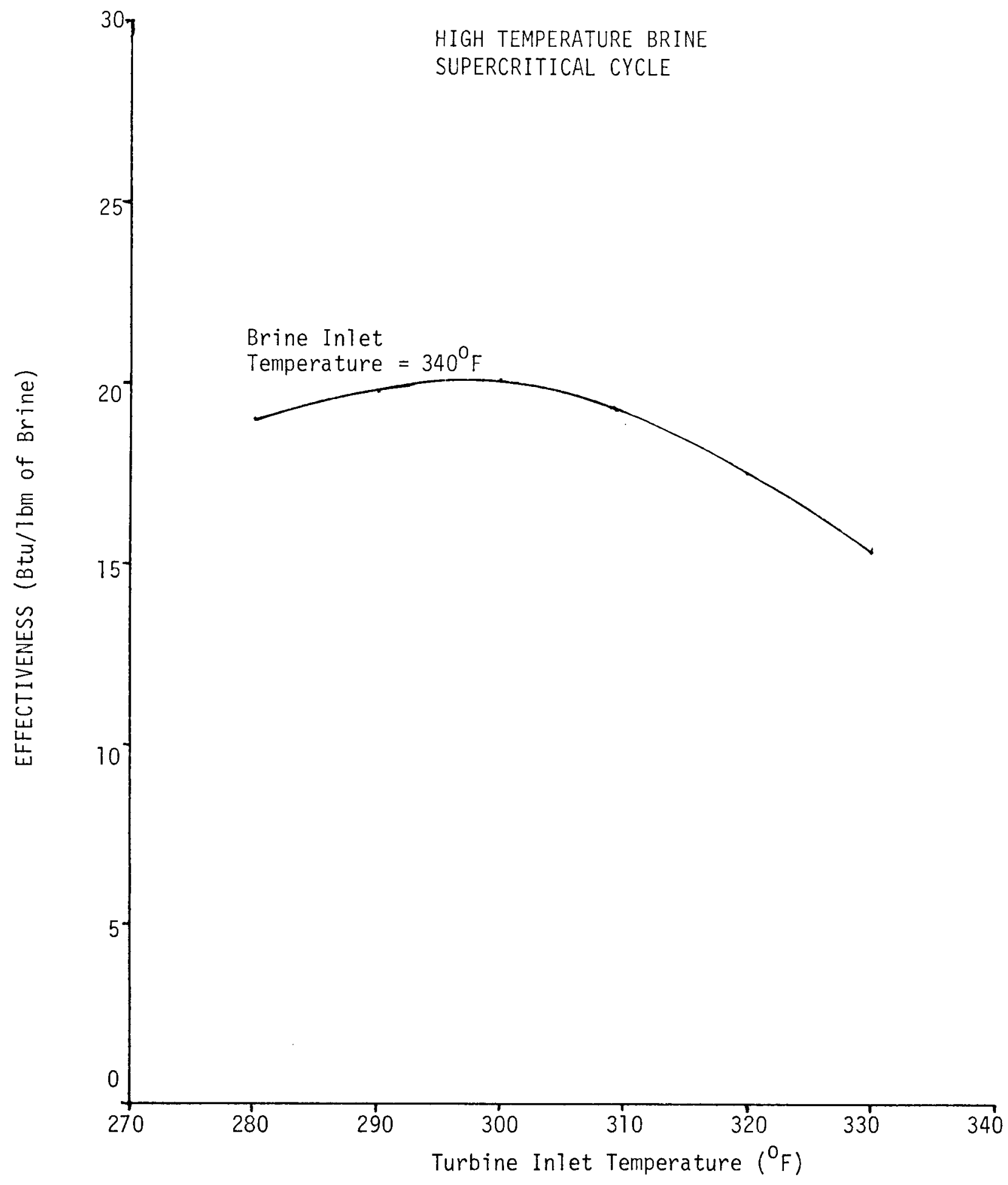

Fiqure 2.3.2-2 Turbine Inlet Temperature vs Effectiveness for the Supercritical Case TB1 - $340^{\circ} \mathrm{F}$ 


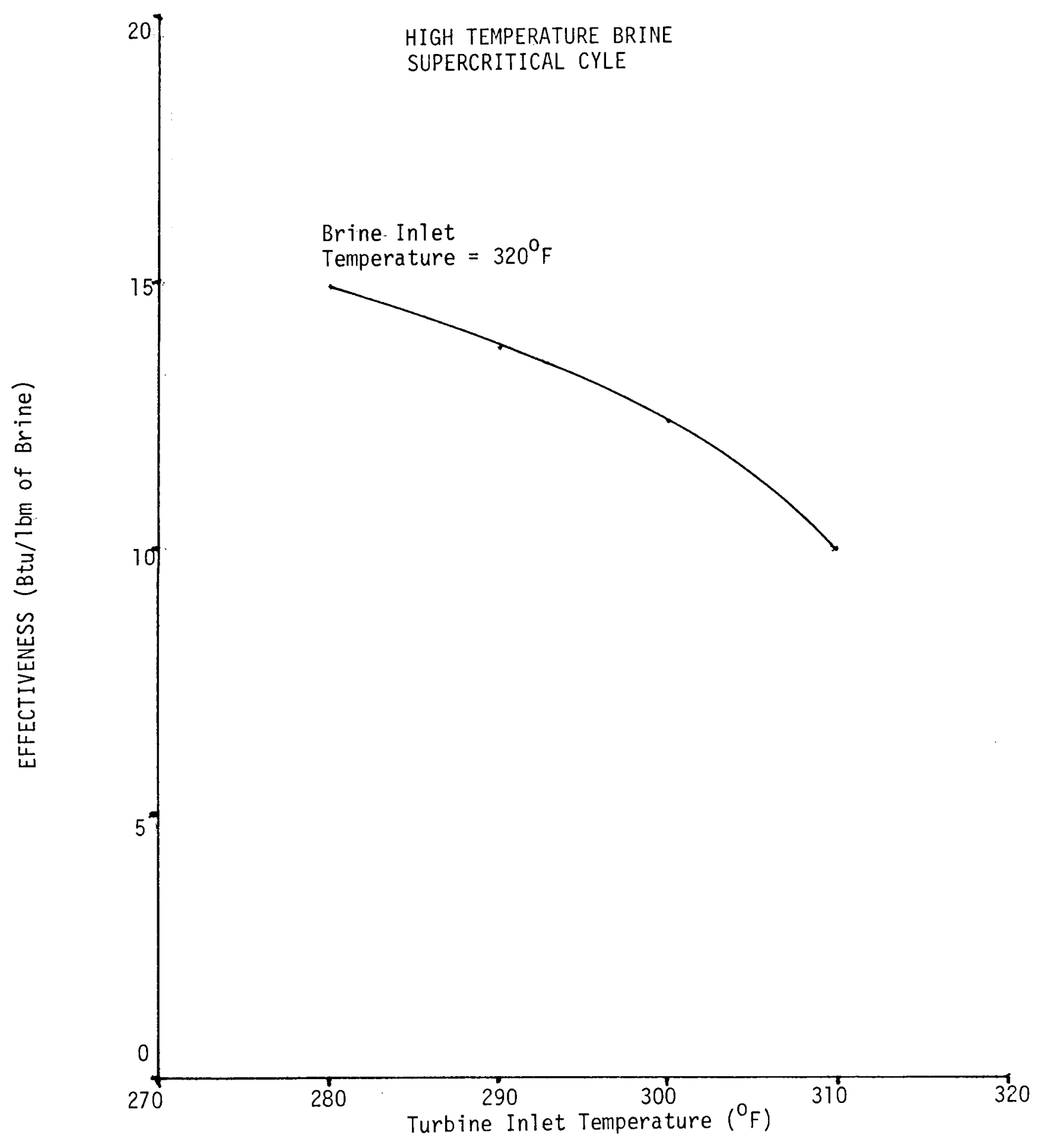

Figure 2.3.2-3 Turbine Inlet Temperature vs Effectiveness for the Supercritical Case TB1 - $320^{\circ} \mathrm{F}$ 


\subsubsection{Double Boiler Cycles}

Double boiler cases with HP boiler temperatures of $270^{\circ} \mathrm{F}, 260^{\circ} \mathrm{F}$ and $240^{\circ} \mathrm{F}$ were run for the high temperature brine cases. The flow spit curves were typified by those in Figures 2.3.3-1, 2.3.3-2, and 2.3.3-3. Figure 2.3.3-1 is the typical flow split curve for a brine temperature of $320^{\circ} \mathrm{F}$, with HP and LP boiler temperatures of $260^{\circ} \mathrm{F}$ and $160^{\circ} \mathrm{F}$, respectively. The maximum effectiveness occurs at a flow split of about 0.14 (both pinch points equal to $10^{\circ} \mathrm{F}$ ), with the effectiveness dropping rapidily as more flow is directed to the LP boiler. This rapid dropoff is caused by the fact that the LP boiler pressure is low enough that the reduction in specific turbine power penalizes the system severely. Figure 2.3.3-2 presents an interesting phenomenon: The maximum effectiveness is found in the single boiler case. Brine temperature is $360^{\circ} \mathrm{F}$, with HP and LP boiler temperatures of $270^{\circ} \mathrm{F}$ and $240^{\circ} \mathrm{F}$, respectively. At the high brine temperatures, the pinch point limitation inherent in medium temperature brine single boiler operation is no longer a problem.

For the medium temperature brine single boiler cases, the brine flow was increased to provide the needed brine energy in the boiler, thereby reducing effectiveness. A second boiler was added to capture the energy rejected at exit of the preheater. More and more flow was diverted to this LP boiler until maximum effectiveness was achieved. For the high temperature brine cases, the controlling factor is not the boiler pinch point, but the LP preheater exit temperature. The pinch point must be increased in order to provide enough energy for the preheater. Therefore, the addition of a second 


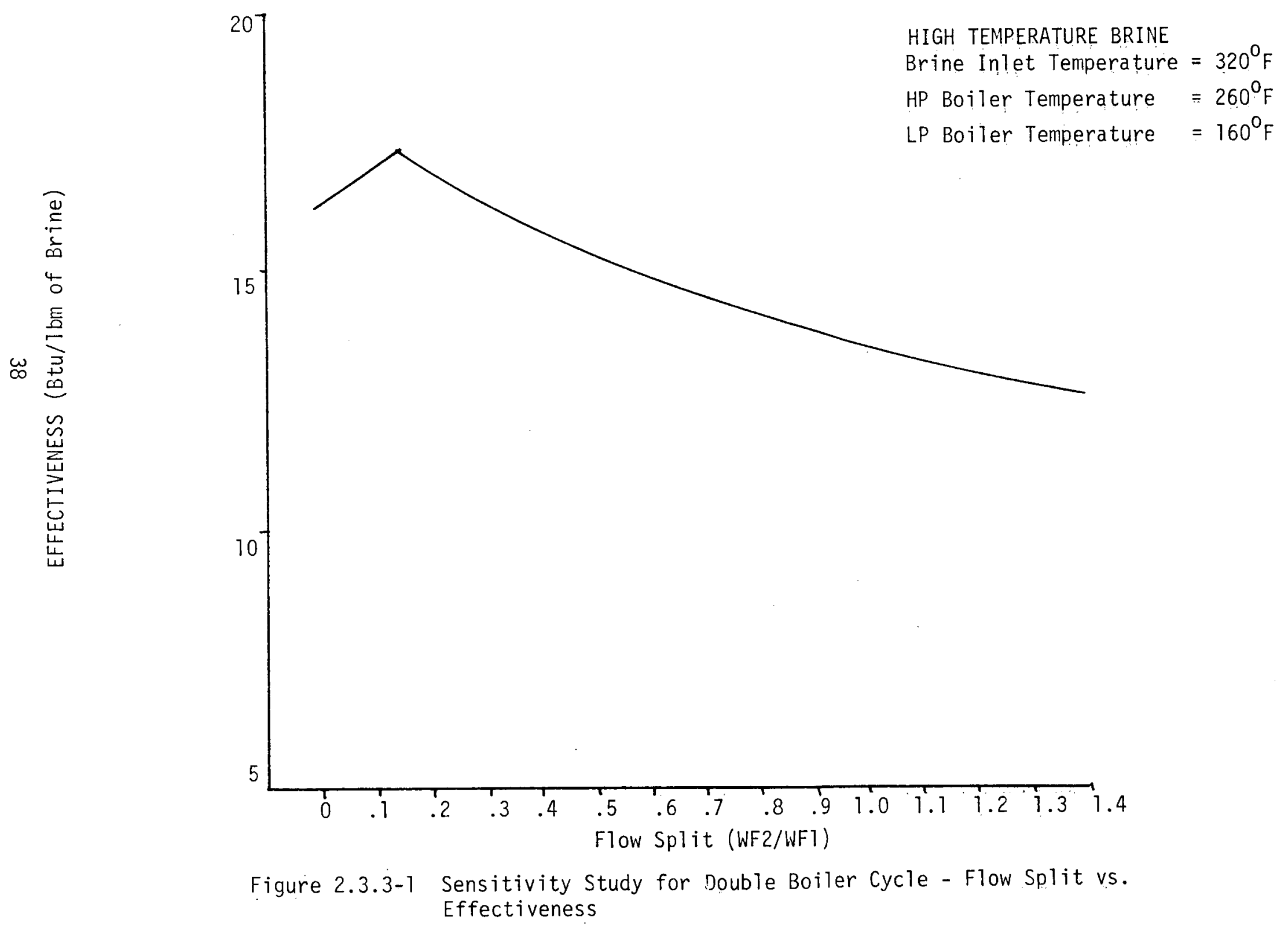




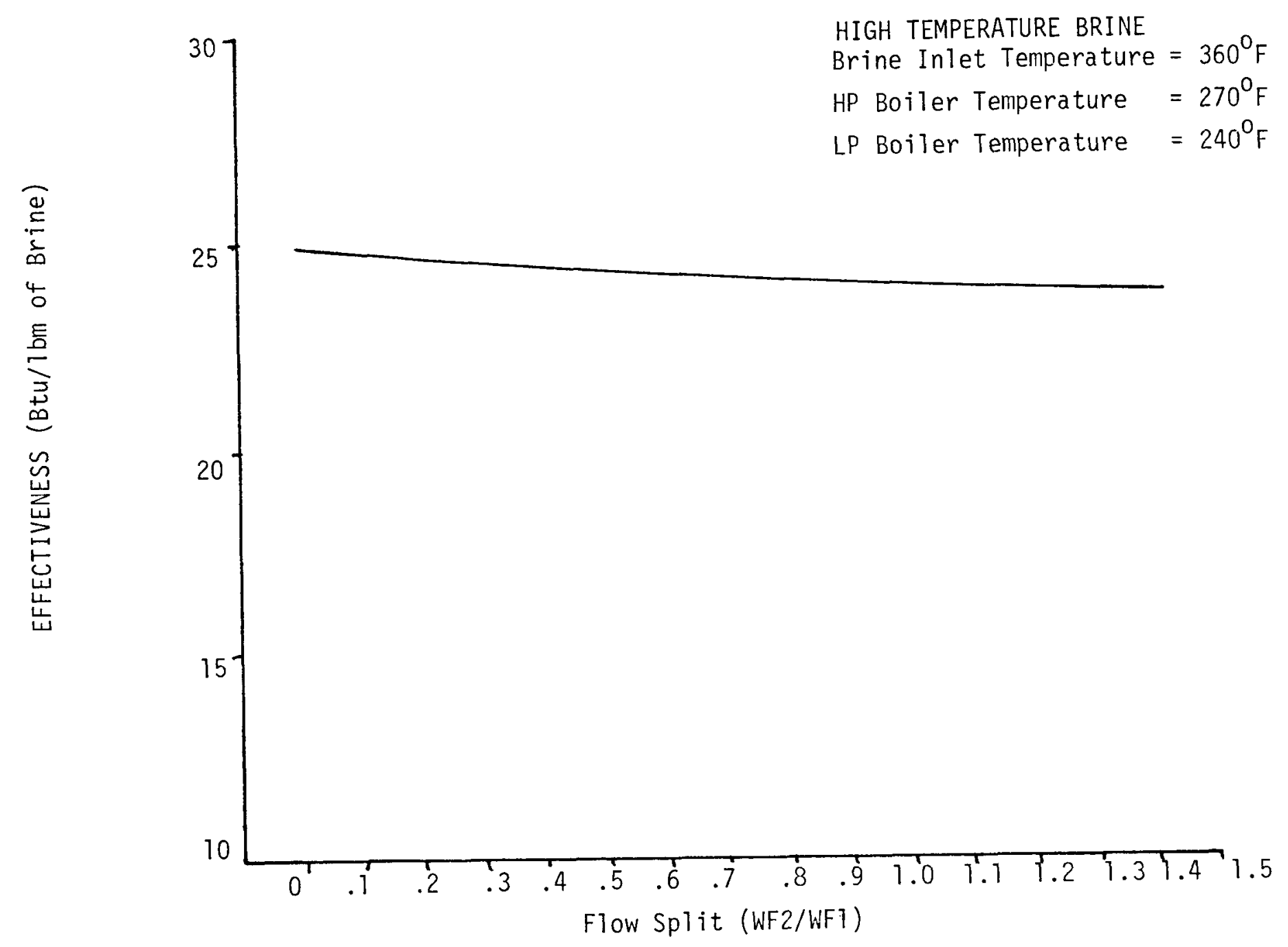

Figure 2.3.3-2 Sensitivity Study for Double Boiler lycle - Flow Split vs. Effectiveness 


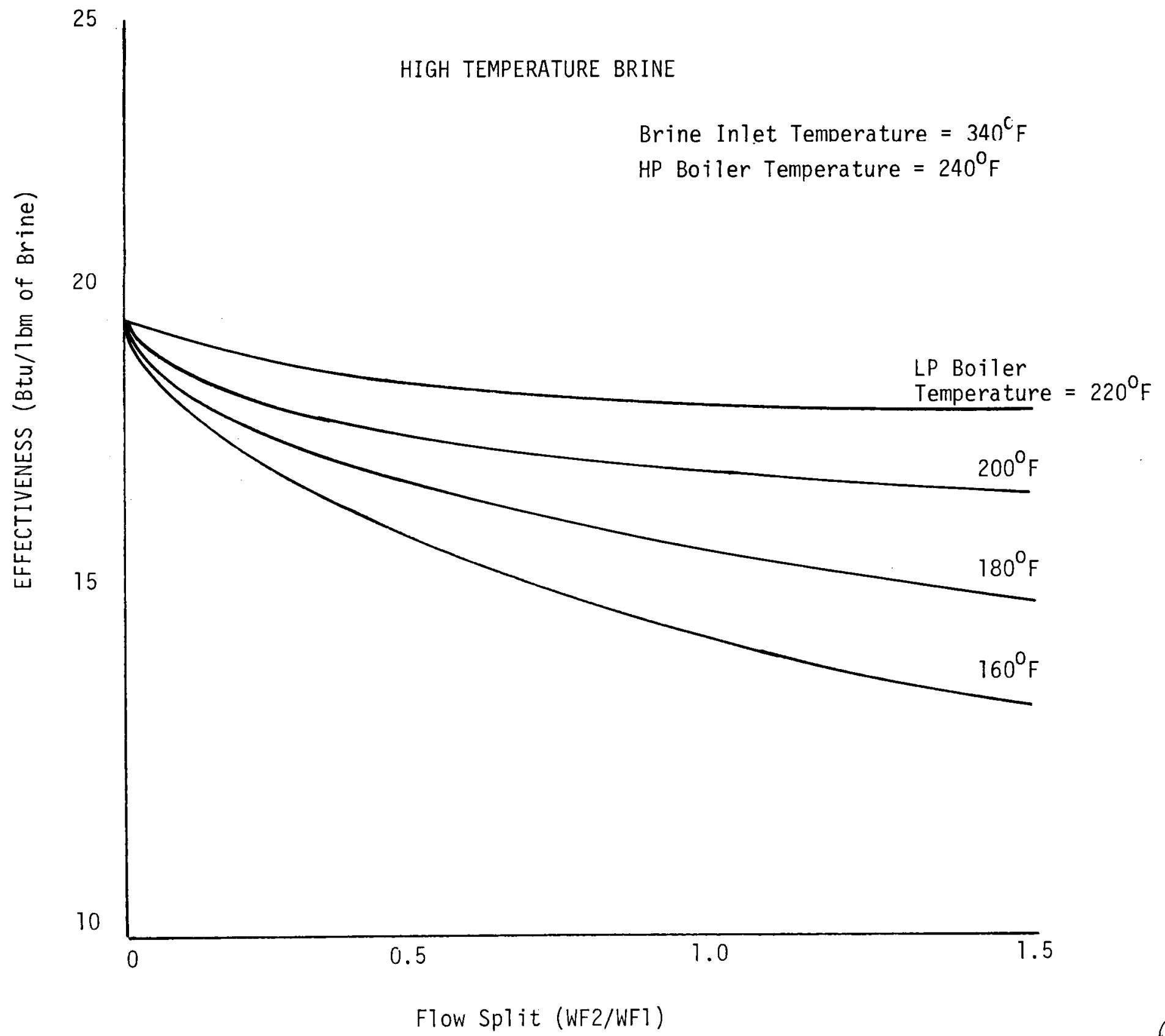

Figure 2.3.3-3 Flow Split vs Effectiveness - 
boiler would accentuate this problem, requiring more brine flow, and lowering effectiveness. For the high temperature brine cases, the LP boiler pinch point defines the brine flow rate, but it must be increased to provide enough brine energy for the LP preheater, which really is the controller. This in turn increases the HP pinch point, indicating that energy is being wasted in that portion of the cycle, too.

Figure 2.3.3-3 is a typical family of flow split curves, produced for the $340^{\circ} \mathrm{F}$ brine temperatures, and $240^{\circ} \mathrm{F}$ HP boiler temperature cases.

Figures 2.3.3-4, 2.3.3-5, and 2.3.3-6 are plots of LP boiler temperatures vs effectiveness. The maximum effective flow split cases for each of three HP boiler temperatures at each brine inlet temperature are plotted. The $340^{\circ} \mathrm{F}$ and $360^{\circ} \mathrm{F}$ brine temperature cases show that the most effective boiler combination is the single boiler case, as discussed above. The $320^{\circ} \mathrm{F}$ brine case curves retain the characteristic shape of the medium temperature brine cases, although the curves are much flatter. 
HIGH TEMPERATURE BRINE

Brine Inlet Temnerature $=360^{\circ} \mathrm{F}$

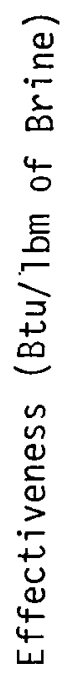

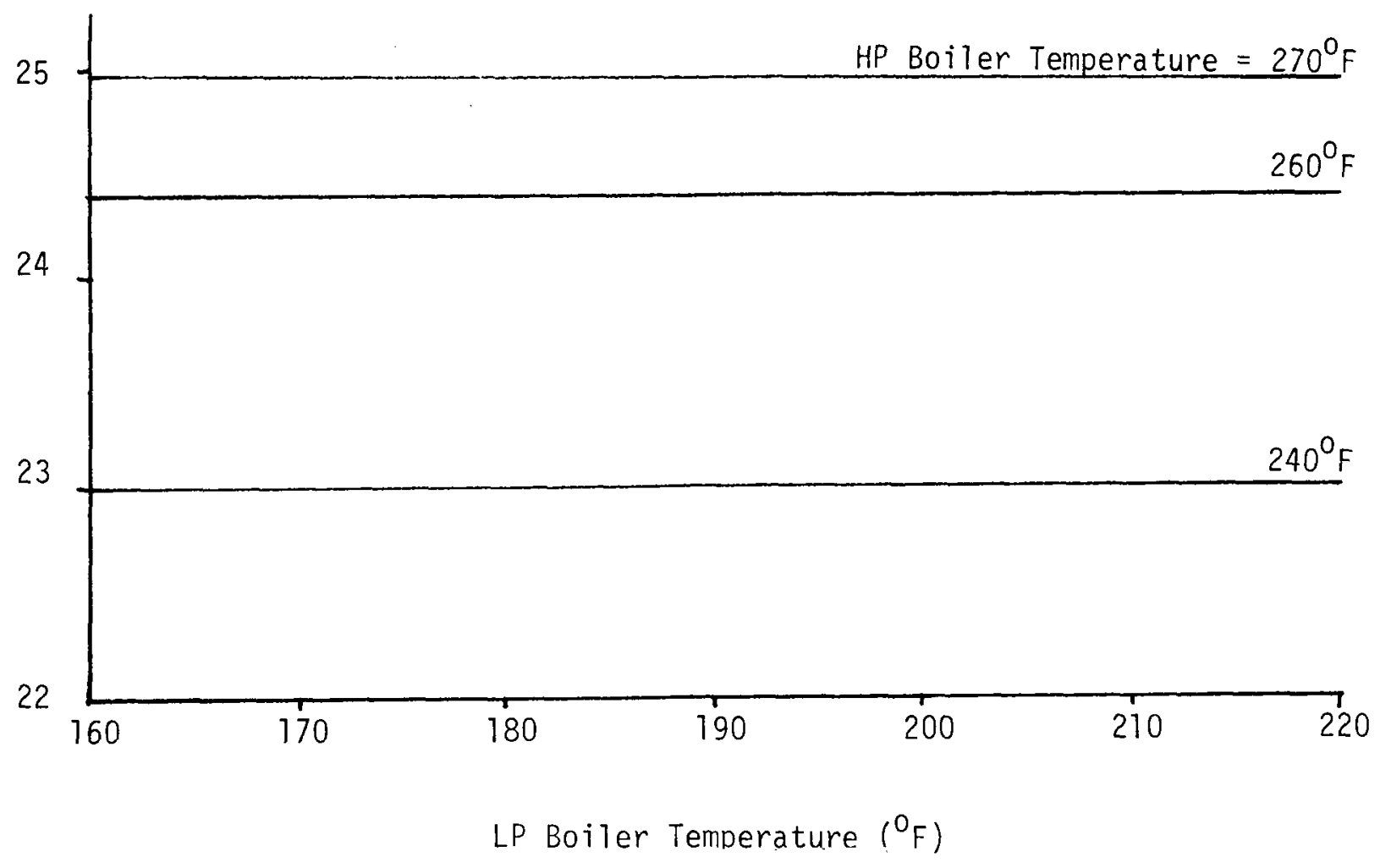

Figure 2.3.3-4 LP Boiler Temperature vS Effectiveness 
HIGH TEMPERATURE BRINE

Brine Inlet Temperature $=340^{\circ} \mathrm{F}$

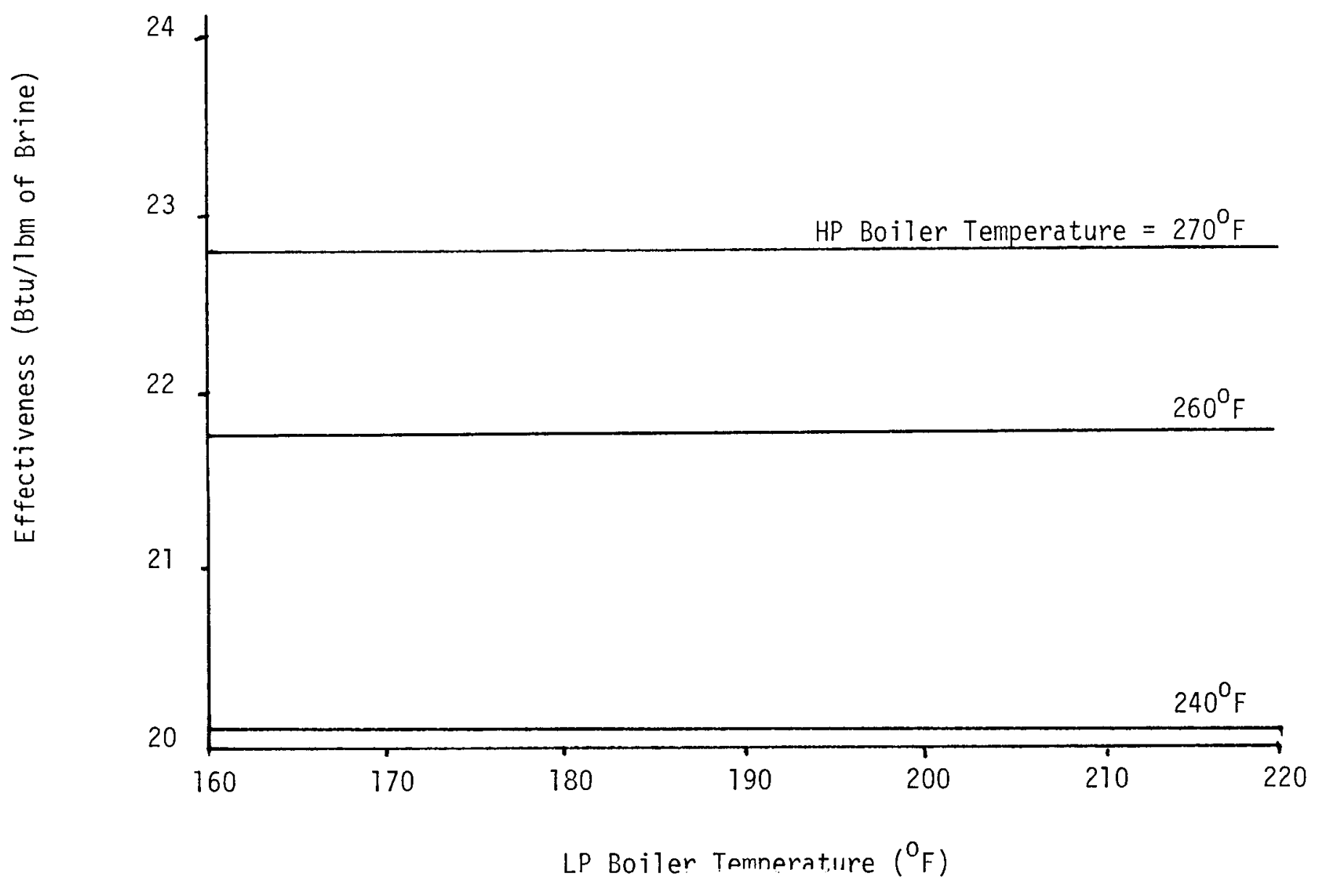

Figure 2.3.3-5 LP Boiler Temperature vs Effectiveness 
HIGH TEMPERATURE BRINE

Brine Inlet Temperature $=320^{\circ} \mathrm{F}$

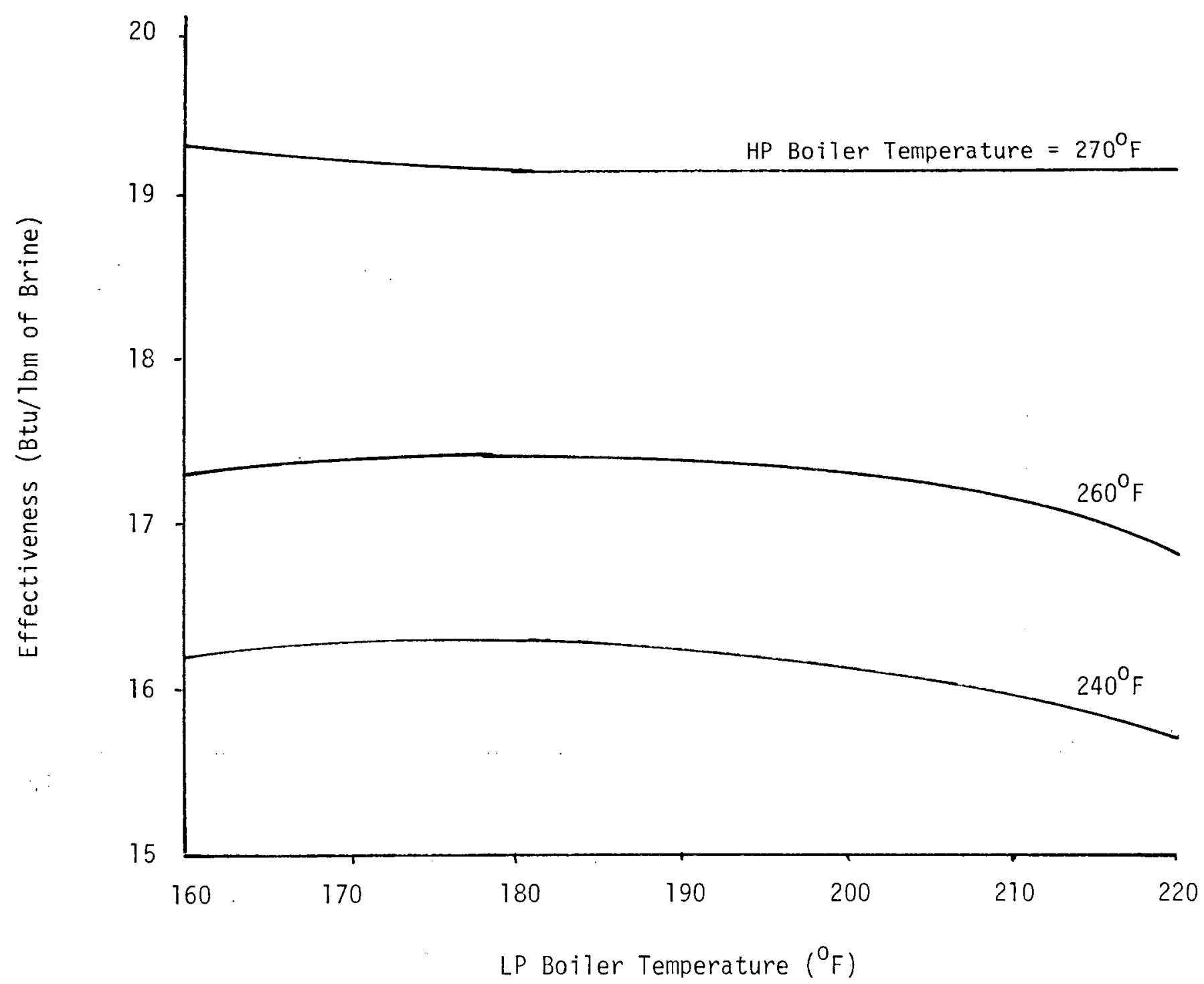

Figure 2.3.3-6 LP Boiler Temperature vs Effectiveness 


\subsubsection{Results of the High Temperature Brine Cases}

For the $360^{\circ} \mathrm{F}$ and $340^{\circ} \mathrm{F}$ brine cases, the single boiler cases were more effective than any of the double boiler combinations for the same HP boiler temperature. The $320^{\circ} \mathrm{F}$ brine cases showed maximum effectiveness at a double boiler cycle, al though the curves are very flat. The single boiler cycles were also more effective than the supercritical cycles for each brine temperature. Figure 2.3.4-1 is a comparison of the single boiler vs supercritical cycles on the basis of turbine inlet temperature. The same data is plotted in Figure 2.3.4-2 on the basis of turbine inlet pressure. The second graph is a good indicator of the pressure range involved. It is the pumping losses at the higher pressures which kill the cycle efficiency. Figure 2.3.4-3 illustrates the increase of isobutane pumping power as turbine inlet temperature increases. Therefore, although the supercritical cycle looks attractive from the standpoint of simplicity, the single boiler cycle is more effective at the high brine inlet temperatures. 


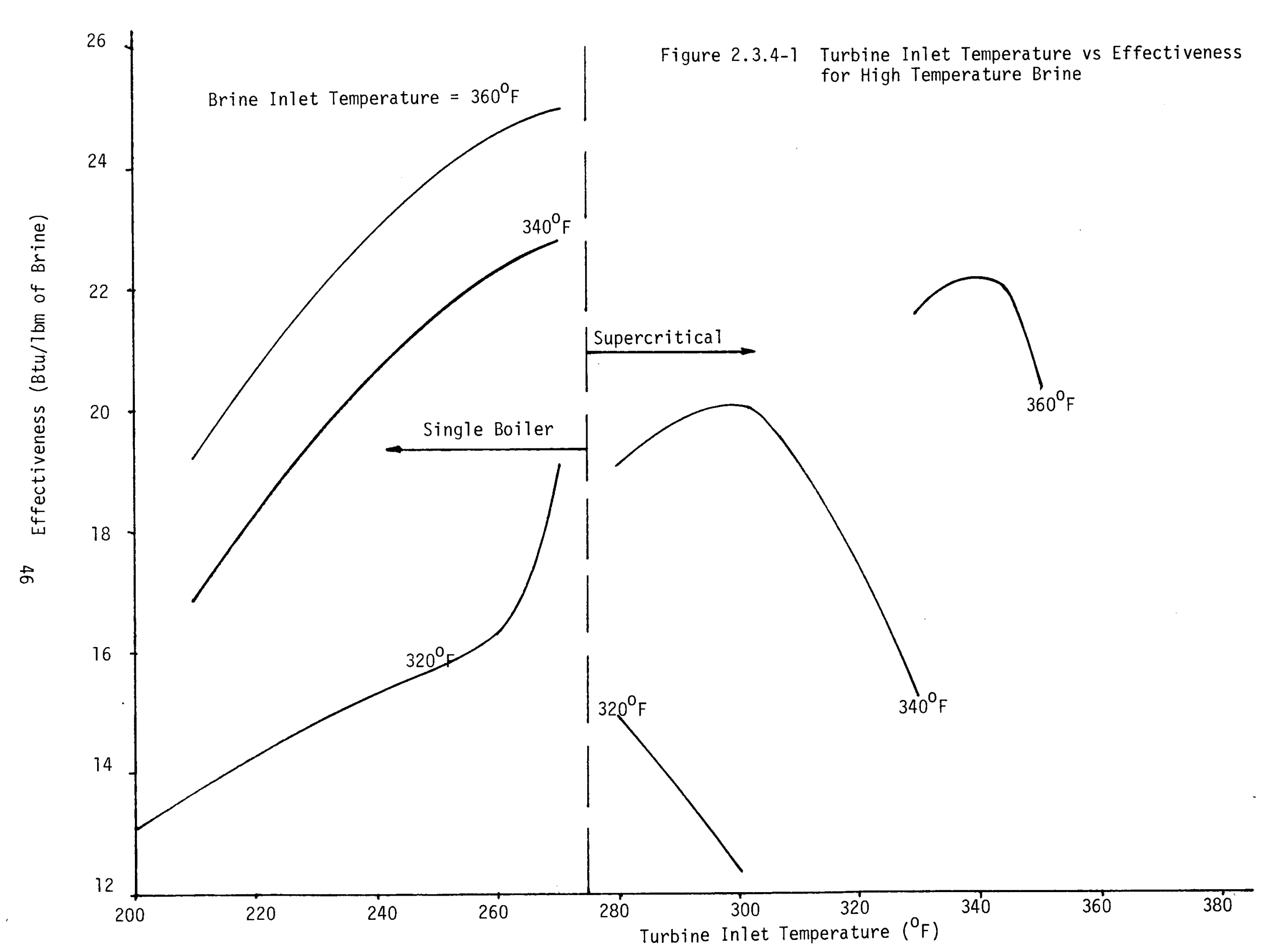

D. . 


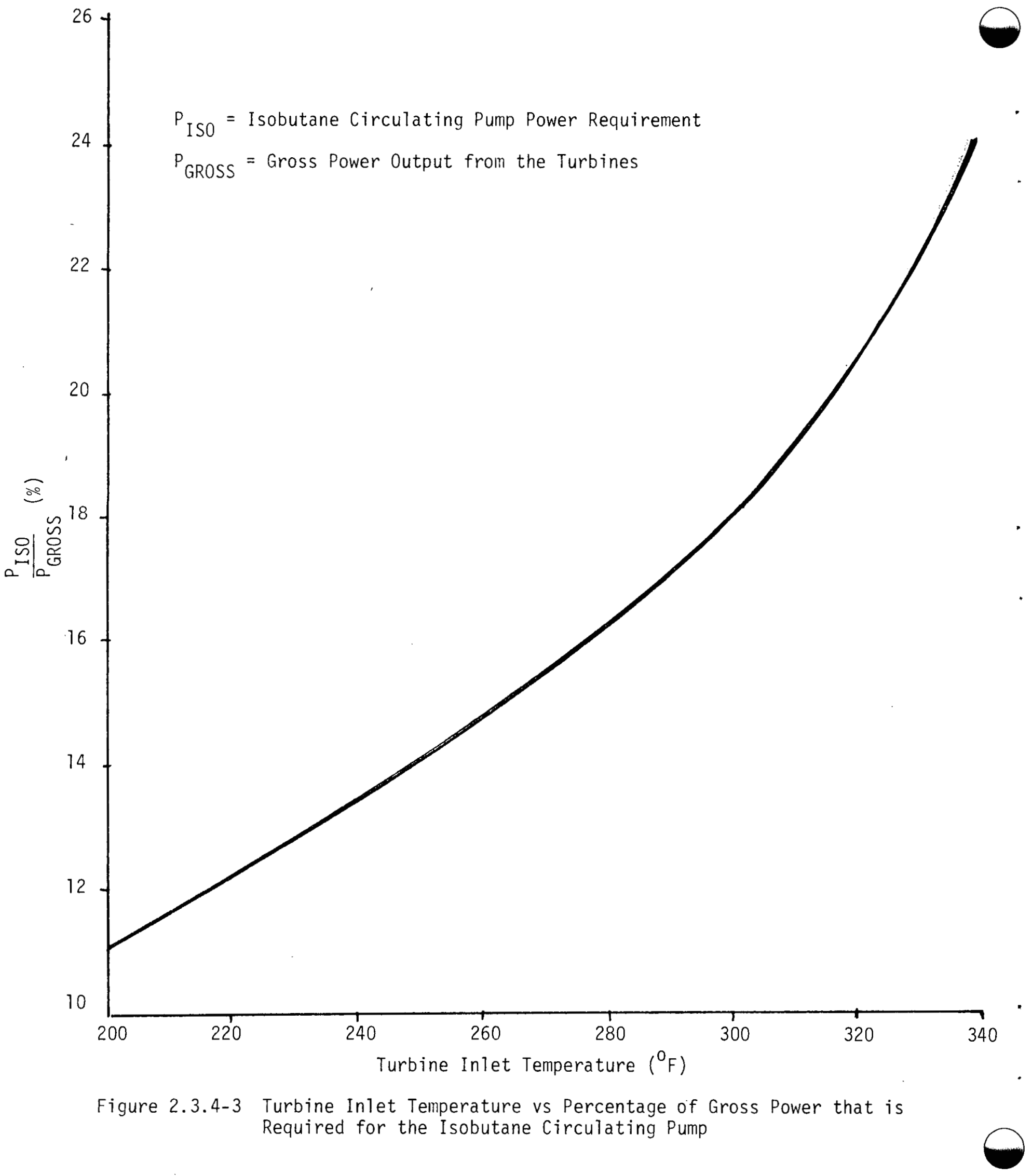




\subsection{Results of the Parametric Study}

In general, five new trends were noted:

1. For the medium temperature brine double boiler cases, the optimum effectiveness was obtained corresponding to a flow split at which both pinch points were minimized. This indicates that, from a thermodynanlic availability stanapoint, both boilers are working equally hard. As the brine inlet temperature increases, the point of maximum effectiveness moves to a lower flow split, indicating that, for the best brine utilization, as the brine temperature increases, more isobutane flow should be directed to the HP boiler. The reason for this effect is found in the pinch point limitations of the boilers. At the lower brine temperatures, the pinch point requirement dictates high brine flow to accomodate one boiler. This results in an elevated brine exit temperature, which reduces effectiveness. If the brine is then cycled through a lower temperature boiler and preheater, this "leftover" energy can be utilized, and effectiveness increases.

2. At high brine temperatures, the point of maximum effectiveness is located at a flow split of zero. In other words, the single boiler case is more effective than any double boiler combination, where the HP boiler is set at the single boiler temperature. This happens because, for a given single boiler energy requirement, the brine flow rate is reduced at high brine temperatures. The pinch point restriction encountered at the medium brine temperatures is no longer a problem, and brine is not exhausted at elevated temperatures. In fact, the brine flow must be substantially increased in order to accomodate a second boiler. 
Since any lower temperature (and pressure) boiler will produce less specific power than the first boiler, effectiveness decreases. Figure 2.4-1 is a graph of the flow split values at which the maximum effectiveness occurs vs brine inlet temperature. The curve indicates that the high temperature brine cases are really a logical extension of the trend noted in the medium temperature brine cases: As brine temperature increases, the point of maximum effectiveness moves to a lower flow split until a flow split of zero (the single boiler case) is reached for the $340^{\circ} \mathrm{F}$ and $360^{\circ} \mathrm{F}$ brine cases. In short, the second boiler is useful only at brine temperatures when pinch point limitations cause wasted brine energy in the single boiler system.

3. Furthermore, at high brine temperatures, the single boiler cycle runs into pinch point problems of a different type. In this case, the brine flow as dictated by the boiler pinch point decreases to the point that there is not enough brine energy to accomplish the preheating. The terminal difference on the preheater actually determines minimum brine flow, the boiler pinch point is increased, and the effectiveness drops.

4. Although brine temperature has a major effect upon the value of the flow split at which maximum effectiveness occurs, there are other factors which also enter the picture. The flow split at which the pinch points are both minimized and control shifts (maximum effectiveness), or the "shift point" is also dependent upon the temperature difference between the brine and the HP boiler and upon the temperature difference between the two boilers. As the temperature difference between the brine and the HP boiler increases (the HP boiler temperature is low relative to the brine temperature), the "shift point" decreases. As the temperature difference between the two boilers decreases, the "shift point" decreases. Therefore, there are three factors 
which affect the flow split value at which effectiveness is optimized; the brine inlet temperature, the temperature difference between the brine and the HP boiler, and the temperature difference between the two boilers.

5. The supercritical cycle was not as effective as the single boiler cycle for the high temperature brine cases. The high pressures required for the supercritical cycle require a large parasitic loss in the form of isobutane pumping power. The increase in turbine output due to the increased pressure ratio is not large enough to offset the loss when the cycle is compared to the lower pressure single boiler cycle. It may be possible to develop a better cycle using regeneration, but for this study, all cycles were formulated to minimize the amount of superheat remaining after the turbine expansion. At any rate, it would appear that for the high temperature brine, another working fluid or fluid mixture may be the answer for a supercritical cycle. 


\section{MAXIMUM EFFECTIVE CASES FOR EACH BRINE TEMPERATURE}

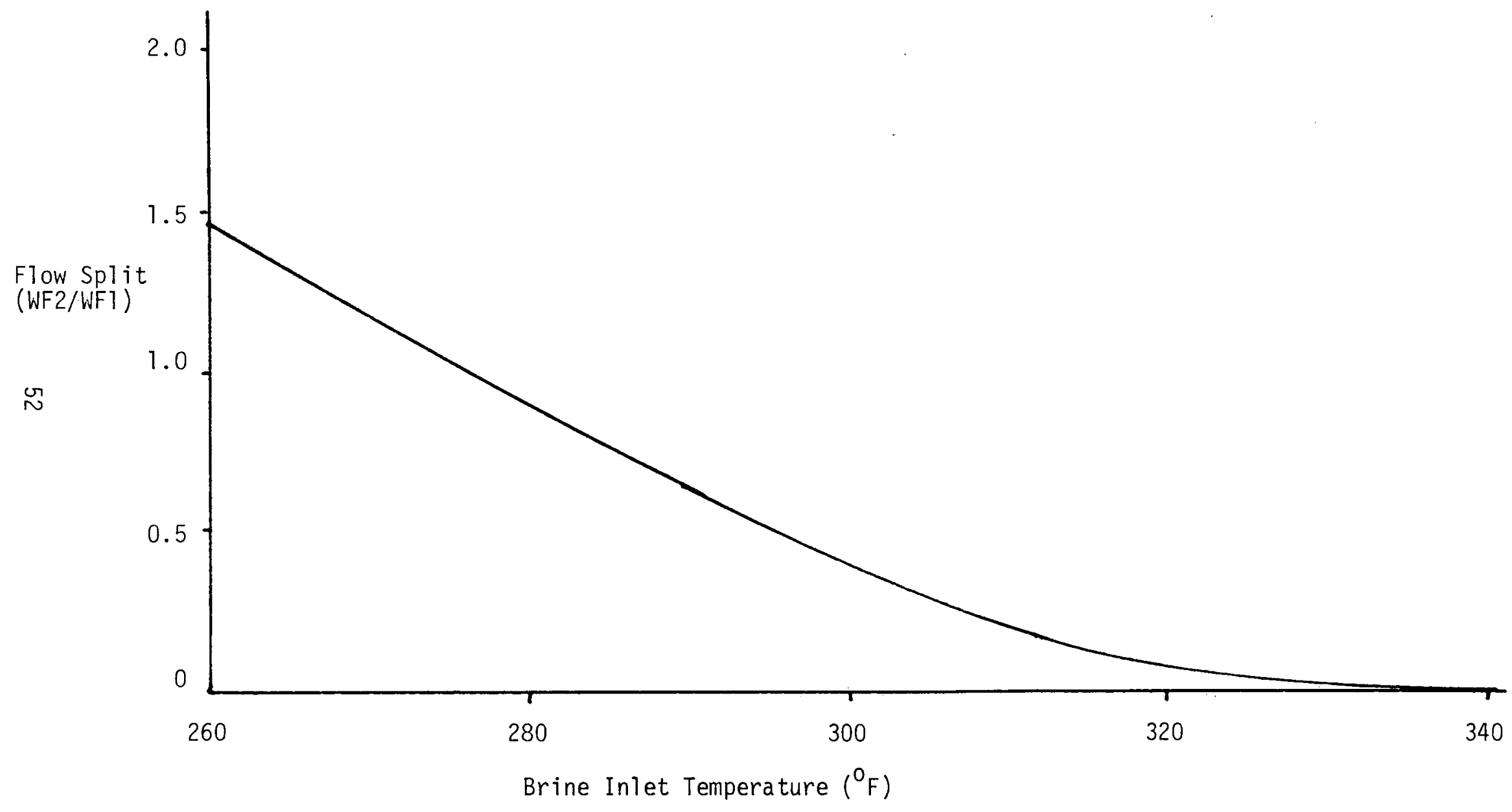

Figure 2.4-1 Brine Inlet Temperature vs Flow Split at which Maximum Effectiveness is Achieved. 
3.0 Determination of the Nominal Design Case (NDC)

The parametric study (Section 2.2) for the $290^{\circ} \mathrm{F}$ brine temperature case was used to determine the optimum thermodynamic cycle for the Nomina? Design Case. The $290^{\circ} \mathrm{F}$ brine case was chosen because the wells at Raft River, Idaho are producing brine at that temperature, and one of the purposes of this study was to determine optimum boiler temperatures for an isobutane cycle power plant to be built at that site.

The Nominal Design Case state point is shown in Figure 3.0-1. The plant is a two boiler system, at $240^{\circ} \mathrm{F}$ and $180^{\circ} \mathrm{F}$, condensing at $105^{\circ} \mathrm{F}$ and producing $5 \mathrm{MW}$ gross or $3.35 \mathrm{MW}(\mathrm{e})$ net. The two boiler system was chosen to alleviate the pinch point problems discussed in Section 2.0. The NDC operates with a flow split of 0.524 at an effectiveness of 10.97 Btu's per pound of brine. 
Binary Cycle, Dual Boiling Geothermal Power Plant

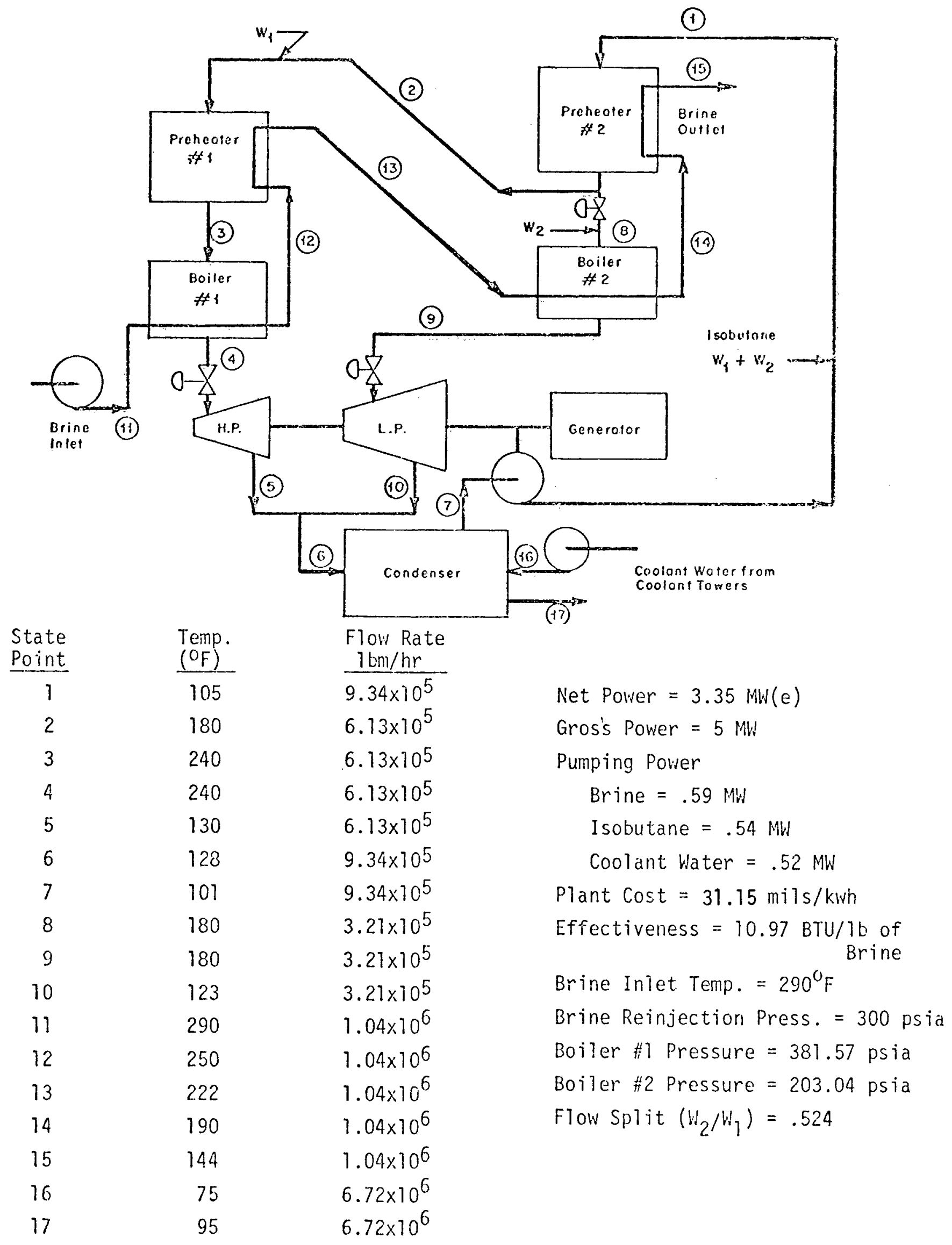

NOMINAL OESIGi: CASE (NDC) 54 Figure 3.0-1 


\subsection{Tolerance Study of the NominaT Design Case}

Several of the parameters input into the computer model are not known exact7y. Therefore a study was performed to determine the effect on plant performance due to the tolerances in the input by using the "floating power" option of GEQSYS. The boiler temperatures and pressures and the heat exchanger areas of the NDC were held constant to simulate the actual plant configuration while each input parameter was varied separately by its tolerance values. This produces a new steady-state case with different effectiveness, power output, flow rates, and brine temperatures. There were four major areas of uncertainty: brine temperatures, condensing temperature, brine-side fouling factor, and specific heats $(C p)$ of the isobutane. Additionally, a separate condenser study was run to determine the effect on plant performance of further lowering the condensing temperature to account for the possibility of lower temperature cooling water being available from the cooling tower during the colder months of the year. Since the tolerance study functions to bracket the normal plant operating regime, and these studies describe another operating condition, they are not actually a part of the NDC tolerance study, but the analysis was performed in the same manner, and therefore is included in this portion of the report. 


\subsection{Brine Inlet Temperature}

The NDC is based upon brine supplied at $290^{\circ} \mathrm{F}$. Since the geothermal wells have not all been drilled, and since they are a fairly new and untried resource, the tolerance on brine temperature was estimated at $\pm 10^{\circ} \mathrm{F}$ or $\pm 3.5 \%$. Running a case at $280^{\circ} \mathrm{F}$, net power was down $4.2 \%$, with effectiveness down $16.4 \%$. Although the total isobutane flow increased by only $4.5 \%$, the flow split shift required to minimize both boiler pinch points increased LP flow $39.9 \%$ and decreased HP flow by $15 \%$. Brine flow is up $15.4 \%$ with a resulting increase in brine pumping power of $14.8 \%$. The brine temperature at the exit of the HP preheater decreased $3.2 \%$, and the brine temperature at the LP preheater exit decreased $2.8 \%$. The reason for these temperature drops is that, for the higher temperature heat source, less brine flow is required, yet the brine temperature at the exit of the boilers remains fixed by the pinch points. This means the brine temperature must be decreased at the heat exchanger exits in order to provide sufficient energy to the isobutane.

Increasing the brine supply temperature to $300^{\circ} \mathrm{F}$ results in a $2.1 \%$ increase in net power with a $16.3 \%$ increase in effectiveness. Total isobutane flow is down $4.6 \%$, with a slightly less severe flow split shift of $-31.5 \%$ on the LP path and $+9.5 \%$, to the HP flow stream. Brine flow is down $12.1 \%$, with an accompanying decrease in brine pumping power of $-12.3 \%$. The brine temperature at the exit of the HP preheater increased $3.2 \%$, and the brine temperature at the exit of the HP preheater is up 2.9\%. Both of these effects are caused by the increased brine flow and increased isobutane flow. The higher flow rate and fixed boiler exit temperatures provide "extra" brine energy at the preheater exits. 


\subsection{Condensing Temperature}

Nominal condensing temperature is $105^{\circ} \mathrm{F}$. Because of uncertainties in cooling tower performance, a tolerance of $\pm 10^{\circ} \mathrm{F}$ or $\pm 9.5 \%$ was placed upon condensing temperature. This indicates a $-13.7 \%,+15.3 \%$ in turbine back pressure. For a condensing temperature of $115^{\circ} \mathrm{F}$, net power decreased $8.7 \%$, and effectiveness went down $10.8 \%$. Total isobutane and brine flow rates were up $2.1 \%$ and $2.9 \%$ with less than $0.1 \%$ change in isobutane flow split. Cooling water flow rate is down $0.7 \%$, with a corresponding $1 \%$ decrease in pumping power requirements. The isobutane pumping requirement is down $0.7 \%$ because the condensing pressure has been raised. Brine pumping power is increased $2.2 \%$, corresponding to the brine flow rate increase. The brine temperature at the exit of the HP preheater is unchanged, but the temperature at the exit of the LP preheater has increased $3.8 \%$ because of increased brine flow.

Condensing at $95^{\circ} \mathrm{F}$ results in a $14.0 \%$ net power increase and a $16 \%$ effectiveness increase. Brine temperatures and all flow rate and pumping power shifts were less than $2 \%$. 


\subsection{Brine Side Fouling Factors}

The HDC brine side fouling factor was $0.003 \frac{\mathrm{hr}-\mathrm{ft}^{2}-{ }^{0} \mathrm{~F}}{\mathrm{Btu}}$. Lack of experience with geothermal brine requires tolerances of $\pm 0.002 \frac{\mathrm{hr}^{-f t^{2}-{ }^{0} \mathrm{~F}}}{\mathrm{Btu}}$ or $\pm 67 \%$.

The overall heat transfer coefficients recalculated corresponding to the minimum and maximum brine-side fouling factors are shown in Table II. The effect of running the plant with a reduced fouling factor of 0.001 is to increase net power by $34.9 \%$. All of the flow rates and pumping power losses are up by approximately the same amount. Effectiveness and brine temperatures remain unchanged since changing the fouling factor only impacts the rate of heat transfer in the heat exchangers and therefore, the amount of flow that can be handled in a heat exchanger of fixed area, with fixed thermal temperature

TAELE II

Overall Heat Transfer Coefficients (U's) Used for the Tolerance Study

\begin{tabular}{|c|c|c|c|}
\hline $\begin{array}{l}\text { Heat Exchanger } \\
\text { Description }\end{array}$ & $\begin{array}{l}\left.\text { NDC U's (Btu/hr-ft }{ }^{2}-{ }^{0} F\right) \\
\text { Brine-side Fouling } \\
\text { Factor }=0.003\end{array}$ & $\begin{array}{l}\text { Mininum U'£ } \\
\left(\text { Btu/hr-ft }{ }^{0} F\right) \\
\text { Brine-Side } \\
\text { Fouling Factor } \\
=0.005\end{array}$ & $\begin{array}{l}\text { Maximum U's } \\
\left(\text { Btu/hr-ft' }{ }^{\circ} \mathrm{F}\right) \\
\text { Brine side Fouling } \\
\text { Factor }=0.001\end{array}$ \\
\hline $\begin{array}{l}\text { HP Boiler } \\
\text { HP Preheater } \\
\text { LP Boiler } \\
\text { LP Preheater } \\
\text { Condenser }\end{array}$ & $\begin{array}{l}157 \\
120 \\
145 \\
110 \\
133\end{array}$ & $\begin{array}{r}97 \\
120 \\
90 \\
112 \\
133^{*}\end{array}$ & $\begin{array}{l}229 \\
158 \\
204 \\
141 \\
133^{*}\end{array}$ \\
\hline
\end{tabular}

* Since the condenser doas not see the brine, changing the brine-side fouling factor does not affect the $U$ value. 
differences. Increasing the brine-side fouling factors to $0.005 \frac{\mathrm{hr}-\mathrm{ft} \mathrm{t}^{2}-{ }^{0} \mathrm{~F}}{\mathrm{Btu}}$ decreases net power by $20.3 \%$, with equivalent decreases in flow rates and pumping power requirements.

Condensing at $95^{\circ} \mathrm{F}$ results in a $14.0 \%$ net power increase and a $16 \%$ effectiveness increase. Brine temperatures and all flow rate and pumping power shifts were less than $2 \%$. 


\subsection{Isobutane Specific Heats}

Specific heat at constant pressure $(C p)$ for isobutane is an input to the program. Without a definitive properties deck, the $C p$ values can vary as much as $\pm 5 \%$. The main impact of this tolerance comes with the results of the parametric study. If the CP's were all input with a consistent bias, the trends produced by the parametric study would still be valid, and the NDC is indeed the optimum operating statepoint. Therefore, the minimum and maximum values of $C p$ were input into the NDC to determine if the effect of varying $C p$ is significant.

Since $\mathrm{Cp}$ varies considerably with pressure and temperature, the input was split into five regions and an average value for each region was used. The five areas are: $C p_{1}$ in the $H P$ superheat region, $C p_{2}$ in the $L P$ superheat region, $C p_{3}$ in the turbine exhaust region, $C p_{4}$ in the condenser region, and $C p_{5}$ in the $\mathrm{HP}$ preheater region. Since superheaters are not being used, $C \mathrm{p}_{1}$ and $C \mathrm{p}_{2}$ do not impact the cycle. With a constant condensing pressure and temperature, $C p_{3}$, and $C p_{4}$ do not vary significantly. Therefore, with the HP and LP boiler temperatures varying $C \mathrm{C}_{5}$ is the parameter which will cause the largest impact, and it was $\mathrm{Cp}_{5}$ which was varied to the tolerance levels.

The effect of varying $\mathrm{Cp}_{5}$ is small and not significant compared to the other, larger uncertainties already present. For $\mathrm{Cp}_{5} \pm 5 \%$, net power is ${ }^{\mp} 0.6 \%$. All of the other plant parameter changes are less than $3 \%$, with the largest being $2.8 \%$ for the LP isobutane flow rate, due to the flow split shift. 
Effectiveness

Net Power

Isobutane HP

Flow Rate

Isobutane LP

Flow Rate

Total Isobutane

a Flow Rate

Brine Flow Rate

Coolant water

Flow Rate

Isobutane Pumping Power

$P$

Brine Pumping

Power

Coolant Water

Pumping Fower

Brine Temp. (A) HP

Preheater Outlet

Brine Temp. a LP

Preheater Outlet
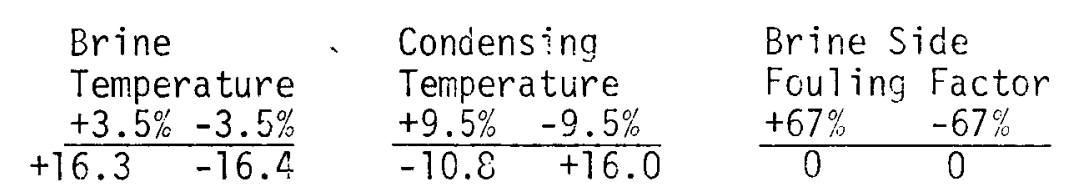

$+2.1-4.2$

$-8.7+74.0$

$+34.9$

$+9.5-14.0$

$+2.1-1.8$

$+34.7-20.6$

$+34.9-20.6$

Total +

$+34.8-20.6$
$+34.8$

$+38.0$

$+35.2$

$+3.3$

$-3.3$

$-2.8+2.9+3.8-3.8$

$+1.3-3.2-0.7+0.4$

$-12.3+74.8$

$-1.0+1.0$

$+34.9-20.4$

$+0.2 \quad-0.4$

$-0.6+0.7$

$+1.5 \quad-1.5$

$+5.0$

$-5.0$

$-20.9$

$-23.9$

$-20.9$

$0 \quad 0$

$\begin{array}{lll}0 & +1.5 & -1.5\end{array}$
$-20.3$

$+1.6 \quad-1.8$

+0 .

$\$ 2.5$

$+0.1-0.3$

$+35.2$

$+38.9$

Tolerances

$\frac{(\%)}{+22.9}$

$+37.67$

$+36.2$

$+53.1$

$+1.9-1.0$

$+0.4-0.1$

$+35.4$

Total -

Tolerances

$\frac{(\%)}{-79.7}$

$-22.5$

$-25.0$

$-37.8$

$-21.2$

$-23.6$

$-20.7$ 


\subsection{Results of the Tolerance Study}

Table III is a summary of the results of the tolerance study. The total tolerance swings on the system were computed by computing the square root of the sum of the squares of the individual component tolerances. All of the positive swings were in one group, and the negative swings in another, regardless of the sign of the input parameter tolerance which caused the plant parameter change.

The total tolerances are dominated by the brine-side fouling factor tolerances, with brine temperature variations also affecting the outcome. Total swing on effectiveness is $+22.9 \%,-19.7 \%$, and $+37.67 \%,-22.5 \%$ for net power. Isobutane LP flow rate exhibited total tolerance of $+53.1 \%,-37.8 \%$ resulting from the variation in flow split necessary to maintain the minimum pinch points. The other flow rates and pumping requirements varied on the order of +35 to $39 \%,-20$ to $25 \%$. Brine temperature variations were sma 11 , on the order of $+5 \%$. Note these total system tolerances do not define additional state points, but the worst combination of tolerance regardless of any interaction effects which may occur if two or more variations happen simultaneously. The NDC state point, with tolerances is shown in Figure $4.5-1$. 


\subsection{Additional Condenser Study}

A study was performed to determine plant performance in case the condensing temperature is dropped below the limits of the tolerance study. The $105^{\circ} \mathrm{F}$ NDC condensing temperature is defined by the minimum cooling water temperature which can be guaranteed year-round $\left(75^{\circ} \mathrm{F}\right)$. This includes the hottest days of the year however, and lower temperature cooling water can be supplied in colder months and at night. Therefore, the NDC was run with condensing temperatures of $95^{\circ} \mathrm{F}, 85^{\circ} \mathrm{F}, 75^{\circ} \mathrm{F}$, and $65^{\circ} \mathrm{F}$, corresponding to cooling water supp $7 y$ temperatures of $65^{\circ} \mathrm{F}, 55^{\circ} \mathrm{F}, 45^{\circ} \mathrm{F}$, and $35^{\circ} \mathrm{F}$, respectively. The floating power option of GEQSYS was utilized, holding ASUM, the sum of the boiler and preheater areas in both loops, constant. In all cases the pinch points were minimized and the flow split remained the same.

Figure 4.6-1 illustrates the variation in the individual heat exchanger areas for the sum of the areas, less the condenser area, held constant. The areas of the LP boiler, of the HP boiler, and of the HP preheater drop $6 \%$ as condensing temperature is lowered from $105^{\circ} \mathrm{F}$ to $65^{\circ} \mathrm{F}$. However, over the same range the area of the LP preheater increases $10 \%$. The decrease in the areas of the two boilers and the HP preheater is due to the decrease in isobutane flow $(6 \%)$ and the corresponding decrease in brine flow (5\%). Isobutane flow decreases because more specific power is produced from each turbine, since the back pressure has been lowered by $47 \%$. The LP preheater area increases, however, because more preheating is necessary to reach the LP boiler temperature from the lowered condensing temperature. Realistically, however, since the areas vary by $10 \%$ or 1ess, they can be considered constant. The condenser area itself is down $26 \%$, because the log mean temperature difference on the condenser has increased from $29^{\circ} \mathrm{F}$ to $41^{\circ} \mathrm{F}$. 
Figure 4.6-2 shows the effects of lowering condensing temperature upon effectiveness and net power output. Net power increases because turbine pressure ratios increase from 4.9 and 2.6 to 9.2 and 4.9 . Therefore more power is produced per pound of isobutane circulated. Effectiveness increases at a faster rate since, at the same time, the brine outlet temperature has decreased from $144^{\circ} \mathrm{F}$ to $122^{\circ} \mathrm{F}$ and more brine energy is utilized. The brine flow is down by $5 \%$. There are three factors which determine the brine flow change. First, total isobutane flow has decreased $6 \%$, therefore there is less working fluid to be heated. On the other hand, as condensing temperature decreases, the isobutane must be heated from a lower temperature, therefore there is an increase in brine energy requirement. Simultaneously, however, since the system sink temperature has been lowered, some of the additional energy requirement can be extracted from brine energy which was previously wasted. The net effect, then, is to reduce brine flow. Since net power is up, and brine flow is down, effectiveness increases a factor of $65 \%$ for a $56 \%$ increase in net power. 


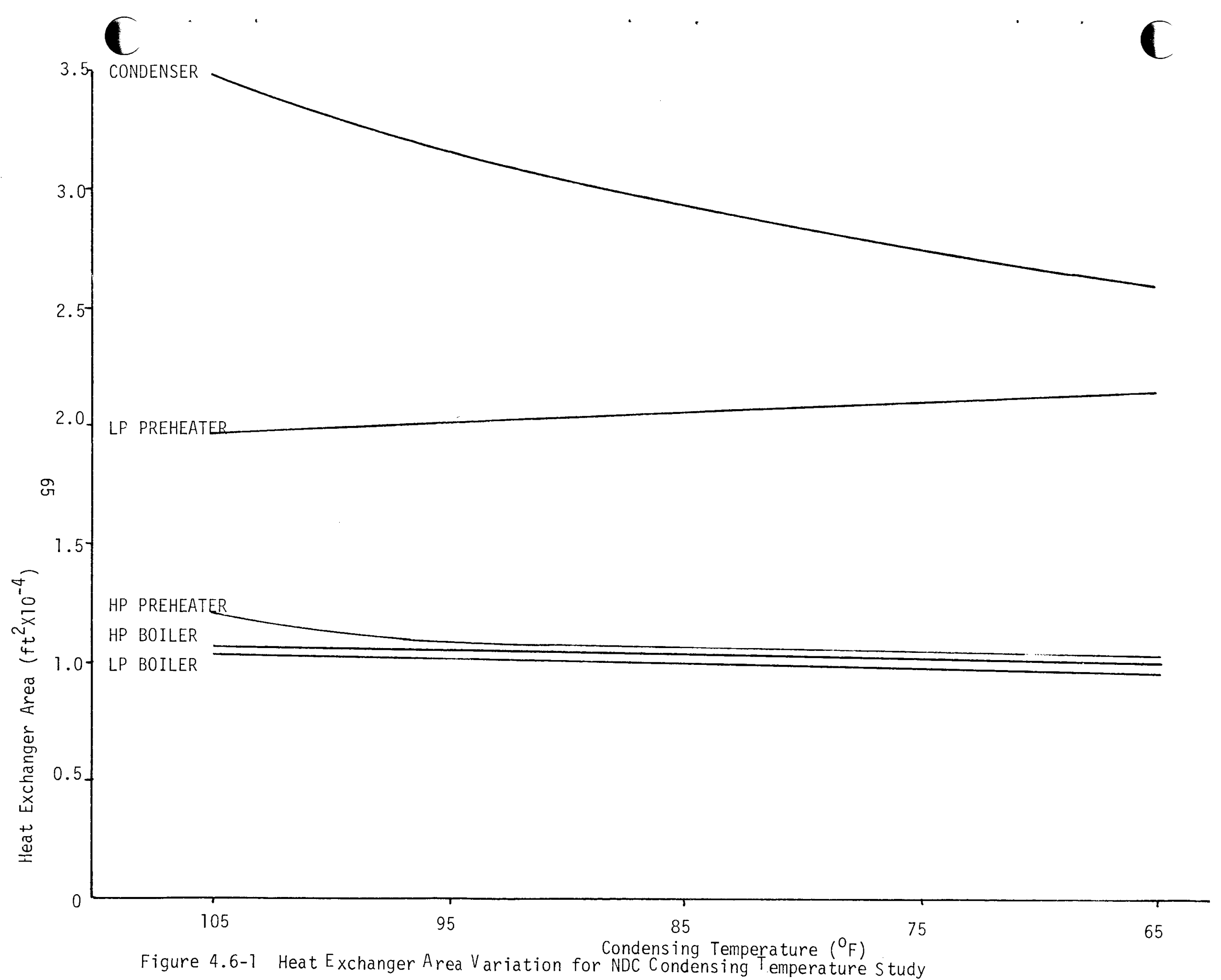




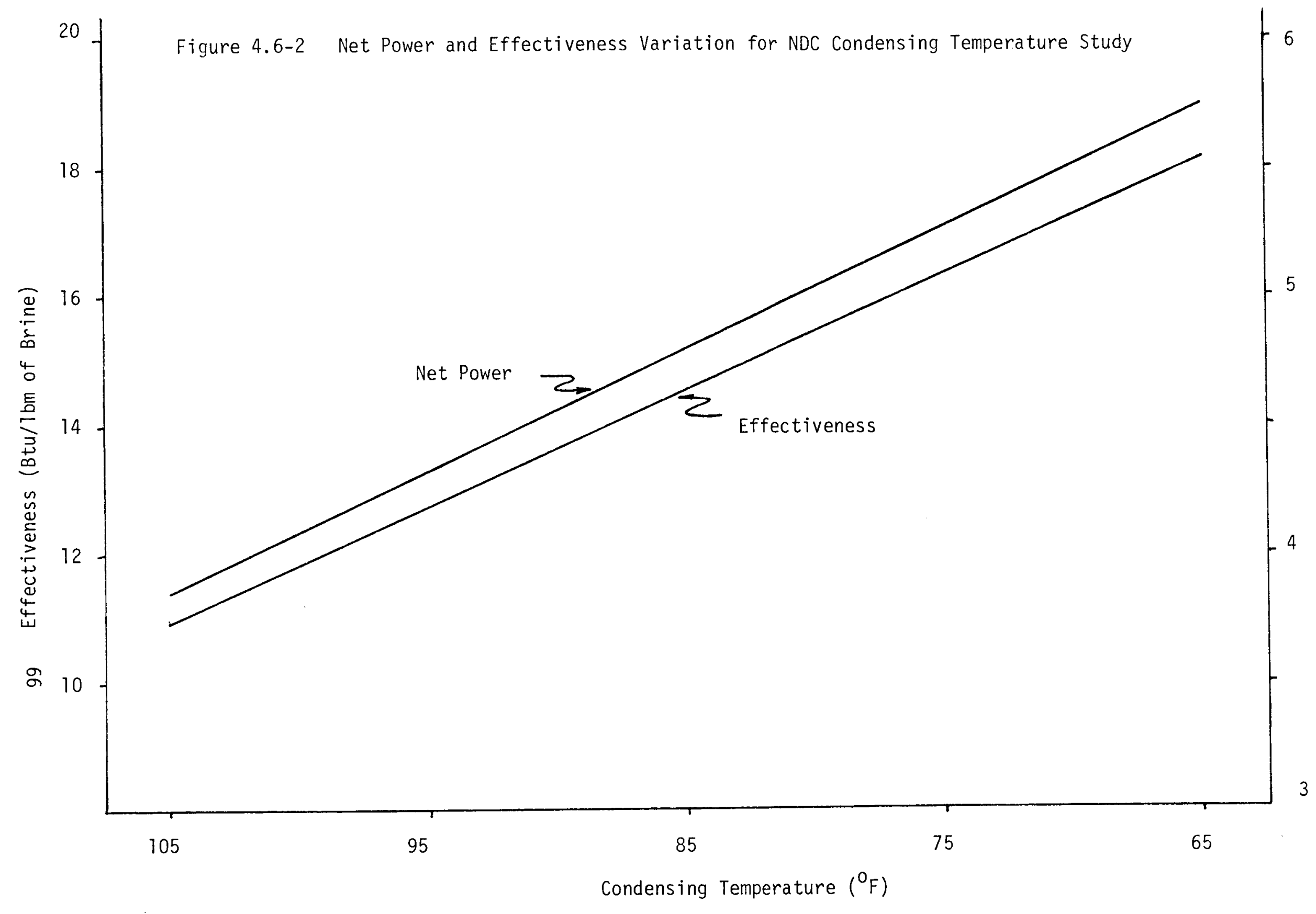




\subsection{Plant cost Studies}

The equations used for determining plant cost were defined in Reference 4. Cost estimates were performed for $10 \mathrm{MWl}$ (e) and $50 \mathrm{MW}$ (e) plants. These were documented in Reference 5. The coefficients and exponents applicable to the $3.35 \mathrm{MW}$ (e) or NDC plant were determined approximately by applying a scaling factor based upon the relationship between the $10 \mathrm{MW}(\mathrm{e})$ and 50 MW (e) plant costs.

The cost of the complete system, TOTSYS, was determined as follows:

$$
\text { TOTSYS }=1.68(\text { EQCOS })+\text { SPYLCO }
$$

EQCOS is the equipment cost, and SPYLCO is the energy supply cost.

$$
\begin{aligned}
\text { EQCOS } & =\text { HEXCOS }+ \text { CDCOS + ELCCOS + SECCOS + TRBCOS } \\
H E X C O S & =\text { heat exchanger cost } \\
& =10.56(A B 1+A B 2)+6.91(A P H 1+A P H 2+A R G+A S H)
\end{aligned}
$$

$A B 1$ and $A B 2$ are boiler areas, $A P H 1$ and $A P H 2$ are preheater areas, $A R G$ is the regenerator area, and ASH is the superheater area. All of these areas are computed in the program.

$$
\begin{aligned}
\operatorname{CDCOS} & =\text { condenser and cooling tower cost } \\
& =7.6(\mathrm{AC})+24.25(\mathrm{WCW}) .65
\end{aligned}
$$

$A C$ is the condenser area and WCW is the cooling water flow rate. Both are computed in the program.

$$
\begin{aligned}
\text { ELCCOS } & =\text { electrical conversion cost } \\
& =(18,835)(\text { PGROSS })(1000) \cdot 42
\end{aligned}
$$

PGROSS is the gross power output in MW of the turbine as calculated in the program.

$$
\begin{aligned}
\text { SECCOS } & =\text { secondary fluid pump cost } \\
& =(2032 .) \quad \text { (PGROSS) }(1000) \cdot 48
\end{aligned}
$$


TRBCOS = turbine-generator cost

$=(8268.5)$ (PGROSS) $(1000) \cdot 53$

SPYLCO $=(.0383)($ WB $)($ PNET $)$

SPYLCO $=(28,400)$ (NOW) $($ PNET)

PNET is the net power output in MW, and NOW is the number of brine wells required. The first equation determines supply cost by brine flow rate, the second by the number of brine wells required. Both were calculated for comparison.

The cost of power was determined thusly:

COSPOW $=\frac{(.03436272)(\text { EQCOS })+(.022968)(\text { SPYLCO })}{(1000)(\text { PNET })}$

COSPOW was calculated for both versions of SPYLCO.

The derivation of these equations is described in detail in Appendix B.

The NDC, at $50 \mathrm{MW}(\mathrm{e})$ net, is fixed with boilers at $240^{\circ} \mathrm{F}$ and $180^{\circ} \mathrm{F}$, condensing at $105^{\circ} \mathrm{F}$. Two factors which affect plant cost are boiler pinch point and flow split. The pinch point impacts cost because as the pinch point decreases from its minimum value, effectiveness increases, and heat exchanger area also increases. As pinch point increases from its minimum, effectiveness starts to decrease, but cost increases due to the increased flow requirements and heat transfer area. Flow split impacts cost because as more flow is channeled to the LP 10op, the specific power produced from the LP turbine is lower, so more isobutane flow is required to produce power. At the same time, overall plant isobutane specific pumping power decreases, since a larger percentage of the flow is pumped to the low operating pressure of the LP loop. At some tradeoff point, the isobutane flow increase is greater than the pumping power decrease and the cost increase.

The NDC was run with varying flow splits and pinch points. Each case produced $50 \mathrm{MW}(\mathrm{e})$ net, with both boilers and condenser operating at the 
NDC pressures and temperatures. The pinch points were varied from $5^{\circ} \mathrm{F}$ to $15^{\circ} \mathrm{F}$. Both pinch points were required to be minimized and equal, and the flow split was calculated to fit these criteria. The flow split was varied from 0.1 to 1.4. Since the flow split was fixed, only one pinch could be minimized (the controlling pinch point) and the other pinch point was allowed to float as necessary. 'In essence, therefore, a new plant was calculated for each case, with different heat exchanger areas and flow rates.

Figures 5.0-1 through 5.0-4 represent curves of effectiveness and cost vs. flow split or pinch point for a $50 \mathrm{MW}(\mathrm{e})$ net plant. Figures 5.0-1 and 5.0-2 represent basically the same information. In Figure 5.0-1, the cost curve is smooth because brine supply cost was calculated on the basis of brine flow rate. This is not a very realistic approach, since a major part of brine cost is well drilling, and it is not possible to drill a part of a well. Therefore, the cost should reflect step changes as additional wells are required. Figure 5.0-2 shows these step changes as the number of brine supply (and reinjection) wells is increased from 20 to 23. It is interesting to note that Figure 5.0-1 indicates minimum cost (based upon flow rate) at a pinch point of approximately $8.5^{\circ} \mathrm{F}$. However, looking at Figure $5.0-2$, an $3.5^{\circ} \mathrm{F}$ pinch point is not minimum, but falls between the two minima of $7^{\circ} \mathrm{F}$ and $10^{\circ} \mathrm{F}$. Figures $5.0-3$ and $5.0-4$ show the relationship between flow split and cost or effectiveness. Figure 5.0-3 represents the brine cost based upon brine flow, and Figure 5.0-4 shows the characteristic step changes in cost when brine supply cost is computed upon the number of wells required. Again, the cost minima are different for the two plots. The effectiveness curve changes as pinch point control shifts with the maximum effectiveness occurring at the flow split. However, the cost curve maximizes at a slightly different flow split. This is due to the fact 
that as flow split changes, pinch points, flow rates, and pumping requirements and specific turbine output for each loop must change. The cost tradeoff point for these factors is close to the effectiveness tradeoff point, showing that effectiveness is a good basis for optimizing the system. 


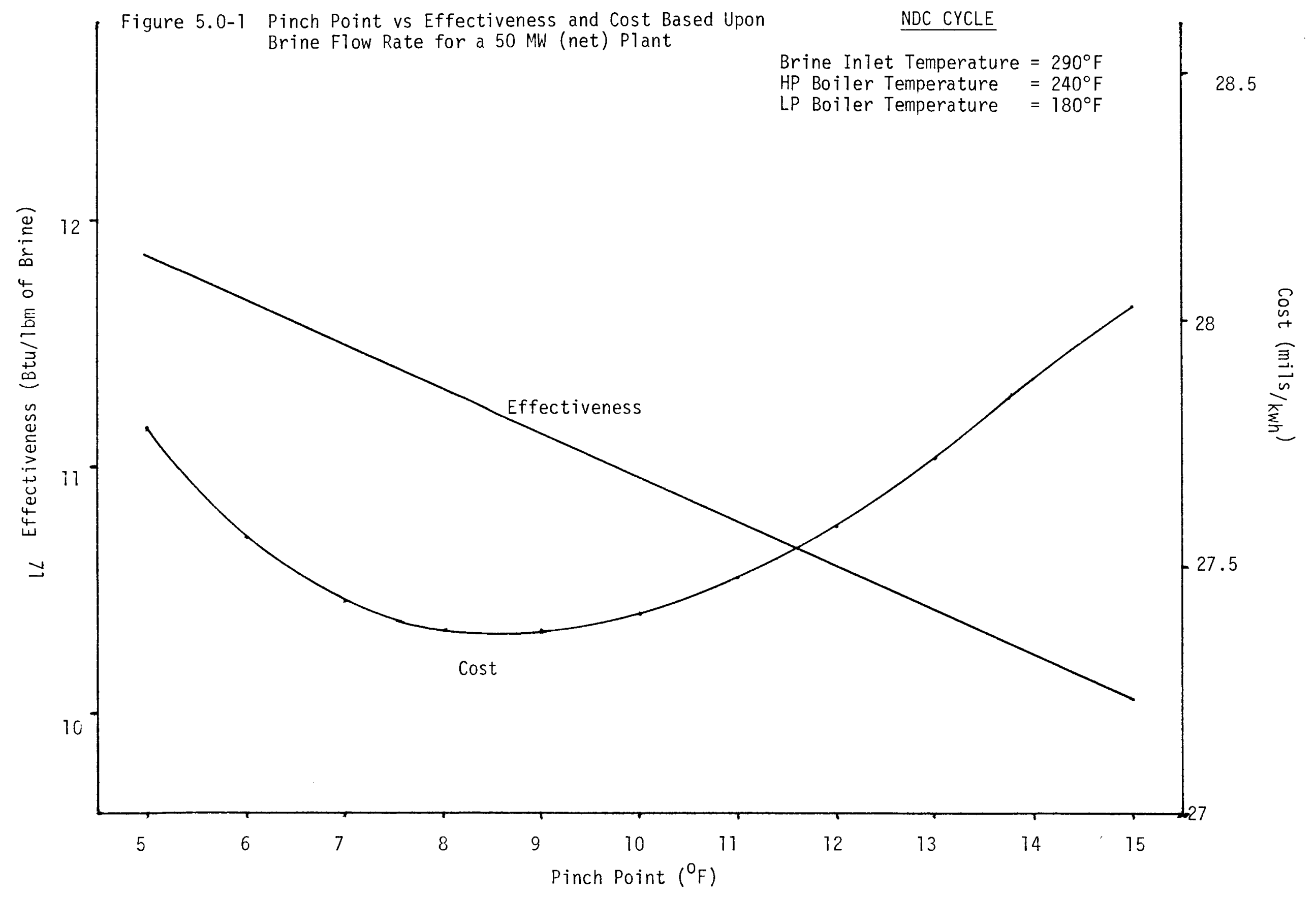




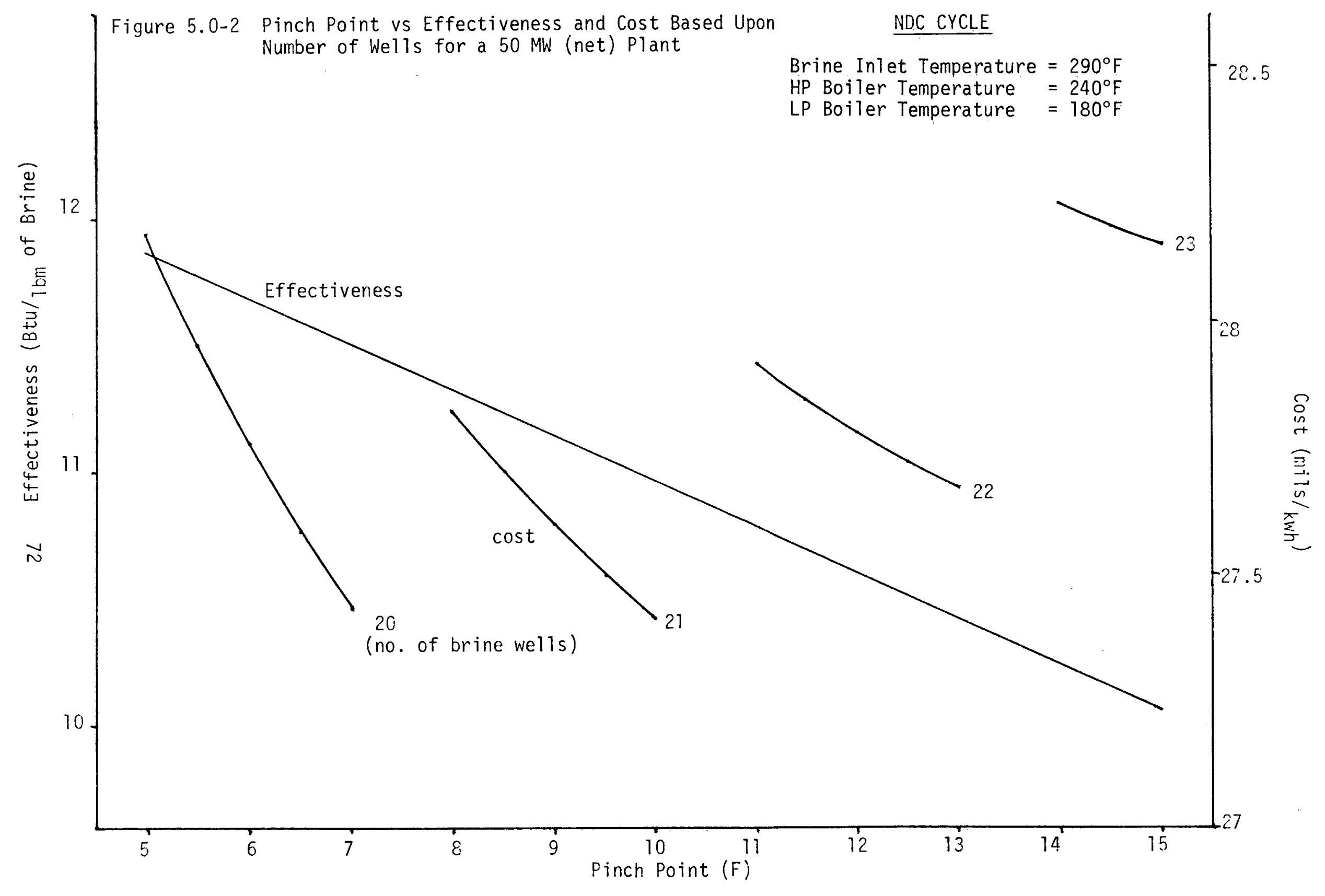




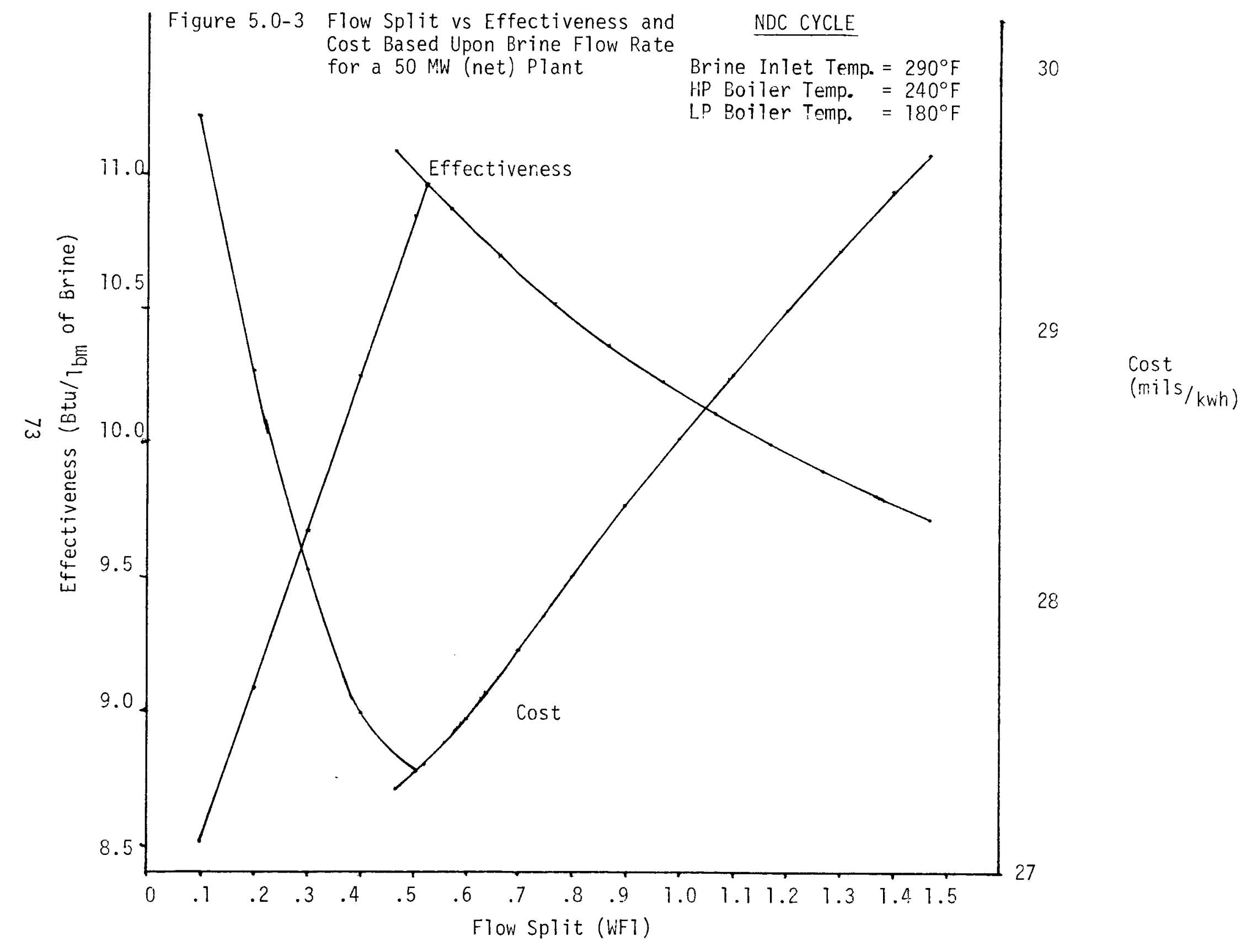


Figure 5.0-4 Flow Split vs Effectiveness and Cost Based Upon Number of Wells for a $50 \mathrm{MW}$ (net) Plant Brine Inlet Temp $=290^{\circ} \mathrm{F}$ HP Boiler Temp. $=240^{\circ} \mathrm{F}$ LP Boiler Temp. $=180^{\circ} \mathrm{F}$

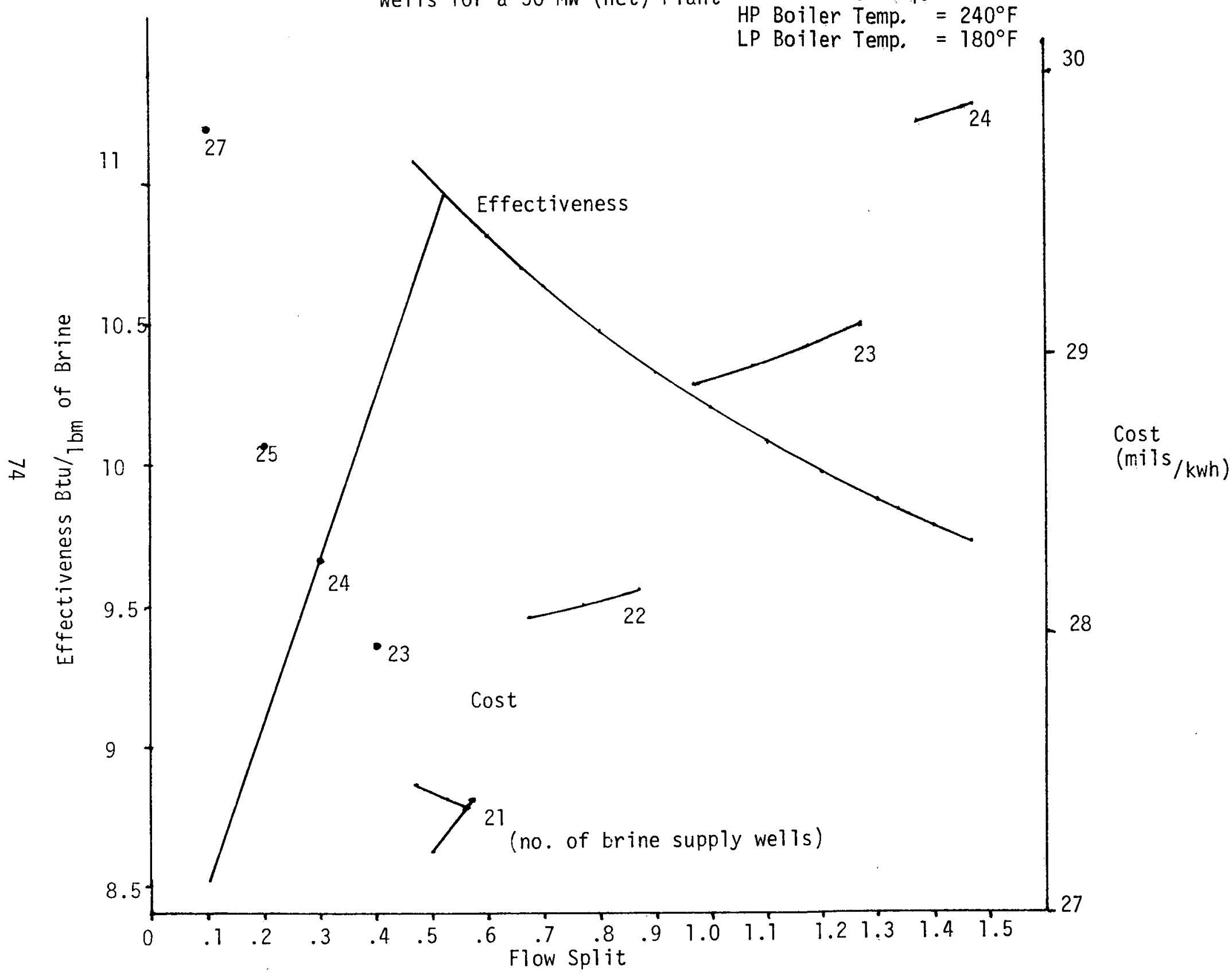




\subsection{References}

1. W. W. Madsen and I. J. Ingvarsson, "Analys is of the Binary Cycle for Geothermal Power Generation," ANCR-1245, December 1975.

2. Letter, G. R. Brown, Uba Heat Transfer Corporation, to R. H. Dart, ANC, April 14, 1975.

3. I. J. Ingvarsson and W. W. Madsen, "Flow Split Sensitivity Documentation Curves for (also RE-E-76-069 Nov. 29, 1976) TREE-1039, Determination of the 5MW Gross Nominal Design Case Binary Cycle for Power Generation at Raft River Idaho.

4. Letter, R. H. Dart, ANC, to W. W. Madsen, ANC, "Power Plant Cost Equations," Dart-4-76, March 11, 1976.

5. R. H. Dart, et. al., "Conceptual Design and Cost Evaluation of Organic Rankine Cycle Electric Generating Plant Powered by Medium Temperature Geothermal Water," ANCR-1226, December 1975. 


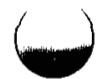

APPENDIX A

GEDSYS

DOCUMENTATION 
I. GE@SYS Proaram Changes - Original Documentation is Found in ANCR-1245

1. Isobutane properties, in the form of a tab deck were incorporated in GEdSYS. These include temperature, pressure, enthalpy, and entropy for the region inside the dome as well as for saturation conditions, superheat, and compressed liquid. These properties were obtained from ASHRAE data, and intermediate values were calculated by linear iriterpolation.

Properties are called by going in with pressure and enthalpy in this manner:

CALL PROPH $(P, H, I)$

PROPH is the properties subroutine which directs the lookup according to $I$. I is a flag from 1 to 9 , which selects the property to be looked up. PROPH first calls POINT, a subroutine which locates the $\mathrm{P}$ and $\mathrm{H}$ in a two dimensional array, and defines the point by means of six coordinates. Four coordinates define the matrix points which bound that value. The other two coordinates are factors which indicate the interpolation distance between those matrix points. All of the property tables are set up so that they correspond to the $\mathrm{P} \& \mathrm{H}$ alignment described above. In this way, the point can be located without searching through the entire property table.

After defining the property in the POINT matrix, PROPH selects the property which is to be looked up by checking the flag, I. I flags the property functions as follows:

I Property to be obtained

1 Temperature

2 Specific Volume and Density

3 Ratio of Specific Heats (dummy values)

4 Specific Heat at Constant Pressure

5 Saturation Properties*

6 Entropy 


$$
\begin{array}{ll}
7 & \text { Thermal Conductivity (dummy values) } \\
8 & \text { Viscosity (dummy values) } \\
9 & \text { Specific Heat at Constant volume (dummy values) }
\end{array}
$$

Each property function calls GRID, which is a linear interpolation function, to locate the property in the array. If pressure and enthalpy are not available an iterative technique is used to locate the property point in order to find enthalpy, then the usual call with pressure and enthalpy is performed.

These are the properties which were formerly input, and which are now called with PROPH:
a) Turbine inlet entropy (HP and LP)
b) Saturated vapor enthalpy at both boiler pressures and at the condensing pressure
c) Latent heat of vaporization for both boilers and for the condenser.
d) Boiler and condenser pressures
e) A one dimensional table of enthalpy and entropy for each condensing pressure to do the turbine expansion

\begin{abstract}
*When $I=5, P R O P H$ is called going in with pressure and temperature in this manner: CALL PROPH $(P, T, 5)$ when $P$ is a dummy variable since temperature defines a constant pressure and temperature line through the dome. The properties which are brought back are the saturated liquid and vapor enthalpies, latent heat, and pressure.
\end{abstract}


2. A subroutine called ITER is used to perform any iterations required.

ITER handles a number of types of iteration. The standard call is $\operatorname{ITER}(I, X, Y I, Y J, F L A G)$ where $I$ is fixed point number indicating the iteration loop number. The deck enclosed was used on a two iteration loop code.

The deck as stands will handle 30 loops. $X$ is the new guess value or initial guess on the first pass, and $Y I$ and $Y J$ are the two values to close on. FLAG is a double precision number which indicates closure. If FLAG is not zero, the iteration is not closed.

In general, ITER uses an initial guess $X$, then $Y I$ and $Y J$ are obtained and stored. The $X$ value is initially bumped by a factor loaded in block data under PCNTI, then new ratios of $Y I$ and $Y J$ are computed. A curve of $X V S$. error is estimated and the point of zero error is extrapolated. The $x$ value is bumped along that curve by PCNTN, which is defined in block data. The program uses minimum and maximum values for $X$ which are input in block data under MINX and MAXX respectively. However, it also sets its own values for each iteration as the iteration progresses. This value can be reset using entry ITERM ( $J, M I N, M A X)$ where $J$ is the iteration number and MIN, MAX the minimum and maximum values of $x$ allowable. The code is set to print if the loop takes more than ten passes to close, but all iterations can be dumped if ISWT(I) is set greater than zero for the particular I iteration loop wanted. If set at zero, it will not dump iterations. ISWT(I) is ordinarily input or specified in the main program. If ISWT(I)=0, MI specifies that the iterations will be printed starting at the MI iteration. NI specifies the maximum number of iterations. Therefore, if ISWT(I)=0 and MI NI, the last iteration of the loop will be printed. EPS in block data is the closure tolerance desired for each iteration. XNAM and YNAM name the iteration loops when they are dumped. 
ICNT and ITF are parameters which may be set to modify the iteration routine if closure is difficult.

In summary, for each application of ITER in a program, ISWT(I) is required in main, and these data are required for the block data program; EPS,MINX,MAXX, PCNTI, PCNTN. Optionally, MI and NI may be changed. They are now set to print the 9 th iteration for a maximum of 9 iterations if ISWT(I) $=0$.

A common example of the use of ITER involves doing an isentropic expansion across a turbine. If a properties lookup is used, going in with pressure and enthalpy, an iterative solution is required to close on entropy. The following series of statements in the main program solves the problem:
a) CALL PROPH(PF2, HI2,6)
b) $S I 2=E N T R$
c) $\mathrm{H} 2 \mathrm{G} 2=\mathrm{HI} 2$
d) 43 CALL PROPHi(PCOND, K2G2,6)
e) CALL ITER( $2, H 2 G 2, E N T R, S I 2, F L A G)$
f) IF (FLAG .NE. O.O) TO TO 43

Statement a) calls the properties deck with turbine inlet enthalpy and pressure to get entropy. Statement b) specifies the entropy to be matched. Statement c) initializes outlet enthalpy with which to enter the properties deck. Statement d) calls the properties subroutine and brings back entropy for the given pressure and the guessed enthalpy (ENTR is brought across in COMMON). Statement e) calls ITER. The 2 indicates that this is a second iteration loop in the program, enthalpy (H2G2) is the independent variable, ENTR is the guessed entropy and SI2 is the value which must be closed on. Note: it is difficult to tell the correct order of $Y I$ and $Y J$. The working solution is to specify the two variables, and if the iteration diverges, to switch their order. Statement f) completes the iteration sequence, since if FLAG does not equal zero, the iteration has not closed. 
ITER is also used to iniprove closure on brine exit temperature and isobutane LP preheater inlet temperature, and to balance the pinch points for the double boiler system. There are three iteration loops which accomplish these tasks. First, the brine temperatures at the entrance of the two boilers is checked with the boiler temperature against a required temperature difference. If the brine temperature is too low, the pinch points are raised, and the brine temperature heat balance is recalculated. The selection of the pinch point to be changed is dependent upon which pinch point is controlling. The program will alternately change both pinch points until the temperature difference requirement is satisfied. Then, the brine temperature at the exit of the LP preheater is conpared to the isobutane temperature at the inlet to the LP preheater. Again there is a temperature difference requirement which must be satisfied. If it is not satisfied, the program changes the controlling pinch point and returns to the beginning to recalculate the brine temperature heat balance. Formerly, GEOSYS performed the pinch point change only by comparing brine exit temperature. This is not a problem with the medium temperature brine cases, since the controlling pinch point could be minimized at the required $10^{\circ} \mathrm{F}$. For the high temperature brine cases, rigidly specifing the $10^{\circ} \mathrm{F}$ pinch point causes the brine flow to drop dramatically. The temperature difference between the brine entering the boiler and the isobutane at the exit of the boiler drops below the value of the pinch point and even becomes negative. This temperature overlap is not reflected in the brine exit temperature and must be checked and corrected in the portion of the program which calculates the brine temperature heat balances. 
3. A new option, to vary flow split, is used for parametric studies. If the flow split is input as 100., the flow split input is varied from -0.1 to 1.6 in 0.1 increments. A new case is computed for each flow split.

4. A plot package was incorporated into GEQSYS. It will plot any variable in the $A(n)$ array vs. any other variable in the program for up to 30 points per plot, and up to 10 plots.

There are three areas which control the plotting routine; two are input, one is program change. The first two input cards of each set of stacked cases must be:

(1) NSUBSC, DEZT in I2,F8.2 format

(2) GRT1TL in 10A4 format

NSUBSC is the number $n$, of the variable in the $A(n)$ array which is chosen to be plotted on the $x$-axis. DEZT is the grid spacing for the $x$-axis. For example, if temperature is to be plotted in $10^{\circ} \mathrm{F}$ increments, DEZT $=10$. GRTITL is the title of the variable on the x-axis. Forty spaces are allocated.

The last card of each case controls the option to plot. The input sequences is: NSTOP, NPLTSW, NCASE, INDEXI, INDEX2, (B(I), I*1, INDEX2) in $3 \mathrm{I} 1, \mathrm{I} 2,1 \mathrm{X}, \mathrm{I} 2,2 \mathrm{X}, 7 \mathrm{E} 10.4$ format NPLTSW and NCASE are flags relating to the plotting routine. The other variables are used to input case data. They will be further explained in the input summary. NPLTSW>0 will dump the data points from the storage array, but the case will be printed as usual. NCASE $=0$ indicates that there are more data points to come for a plot. The next sets of case cards should not have the two NSUBSC, DEZT and GRTITL cards in front until there is a card with NCASE>0. This says that all of the previous stored points from the last set of NSUBSC, DEZT and GRIITL cards up to and including the current point are to be plotted on one graph. It also indicates that the next case will be prefaced with NSUBSC, DEZT and GRTITL cards, indicating that a new set of points for another plot will be generated. 
The $A(n)$ array variable is specified and named in the input as above, but chosing the other program variabie requires a program change in these cards:

RATPLT(NPOINT) $=$ variable name to be plotted on the $y$-axis

CALL TITLE $G(Z, 40$, GRTITL, NY, YNAME, NT, TNAME $)$

NY is the number of spaces for the Y-axis title (up to 40). YNAME is the $\mathrm{Y}$-axis title. NT is the number of spaces for the plot title (up to 4.0), which will be centered beneath the $x$-axis title. TNAME is the plot title. 
II. Input to the Program

A. First set of cards: Plotting information, describing the member of the $A(n)$ array which is to be plotted on the $X$-axis, the grid spacing, and title for the $X$-axis. These cards are required at the beginning each time the program is run, and in order to initiate a new plot.

B. Second set of cards: Case Information

1. First Card: Title card containing any pertinent information.

2. Second Card: Heat exchanger data. This card flags the option for closing on heat exchanger areas and varying the net power output. The format is $(7 X, E 10.4, I 5)$. The heat transfer area is swuare feet is input in E10.4. IFLG is input in I5. This flags which area is to be kept constant

$$
\begin{aligned}
\text { IFLG }= & \text { - flag out this option } \\
& 1 \text { - flag out this option } \\
& 2 \text { - total heat transfer area } \\
& 3 \text { - preheaters, boilers and superheaters } \\
& 4 \text { - HP boiler } \\
& 5 \text { - LP boiler } \\
& 6 \text { - HP preheater } \\
& 7 \text { - LP preheater } \\
& 8 \text { - condenser } \\
& 9 \text { - regenerator }
\end{aligned}
$$

3. Third Card: starts data set for the case. For the first case, all 50 data must be input. The first column of a case data card is NSTOP, in I1 format, which calls several options: NSTOP $=0$ Go on to read the next card

$=1$ Placed on last card of last case run to indicate the end of case data. 
$=2$ Placed on last card of a base case. All information input for a base case will be saved until it is replaced by another base case.

$=3$ Placed on the last card of a stacking case. The information contained in the stacking case cards will replace base case information for one run only, then will be scratched, and the base case information is retrieved.

$=4$ Placed on the last card of a supercritical case. This flags the supercritical oftion in the program.

$=6$ Placed on the last card of the last supercritical case run to indicate the end of case data, as well as to flag the supercritical option.

The next two columns contain NPLTSW and NCASE to control the storage of data for plotting and to call for new NSUBSC, DEZT, and GRTITL cards and a new plot

These are followed by an index number (INDEX1) in the 12 format. The input parameters are placed in an array, numbered 1-50. The index number is the array number of the first data peice on the card. INDEX 1 is followed by two spaces, then INDEX2, the number of pieces of input data following on that card is read in (12 format). Starting in column 11, data is input in E10.4 format. Therefore 1 to 7 pieces of consecutively numbered data may be read on one card. 
INPUT PARAMETERS

( $A(n)$ Array Variable Nlames)

Variable

Name

1. TF1

2. TF2

3. $\mathrm{PFI}$

4. DUMMI

5. DUMM2

6. DUMM3

7. DUMM4

8. DUMMI5

9. TCOND

10. VFCOND

11. PCOND

12. HFCOND

13. $\mathrm{CPFI}$

14. XK

15. $\mathrm{CONDH}$

16. TB1

17. DLTPPI

18. DLTPP2

19. DLTSC

20. DTRG

21. $\mathrm{CPB}$

22. NP

23. TCWO
Definition

HP boiler temperature

LP boiler temperature

HP boiler pressure

ISWT(1) for ITER print option loop $1>0$

ISWT(2) for ITER print option, loop $2>0$

ISWT(3) for ITER print option, Loop $3>0$

ISWT(4) for ITER print option, Loop $4>0$

ISWT(5) for ITER print option, loop $5>0$

Condensing temperature

Liquid specific volume a TCOND

Dummy input

Dummy input

Cp @ HP turbine inlet

Ratio of specific heats

Dummy input

Brine inlet temperature

HP boiler pinch point

LP boiler pinch point

Degrees of subcooling in condenser

Minimum terminal difference required for a regenerator

$C p$ of the brine

Pump efficiency,

Coolant water temperature at condenser exit

$40 \mathrm{~F}$

0.9
NDC

Value

$240^{\circ} \mathrm{F}$

$180^{\circ} \mathrm{F}$

381.57 psia

\section{0} 0 $>0$ 0 $1050 \mathrm{~F}$

$0.03 \mathrm{FT}^{3} / \mathrm{LB}$

$0.63 \mathrm{BTU} / \mathrm{LB}-{ }^{0} \mathrm{~F}$

1.11

$290^{\circ} \mathrm{F}$

$10^{\circ} \mathrm{F}$

$10^{\circ} \mathrm{F}$

$10^{\circ} \mathrm{F}$

1 BTU/LB-OF

$950 \mathrm{~F}$ 


\begin{tabular}{|c|c|c|c|}
\hline & $\begin{array}{l}\text { Variable } \\
\text { Name } \\
\end{array}$ & Description & $\begin{array}{c}\text { NDC } \\
\text { Value } \\
\end{array}$ \\
\hline 25 & TCWIN & $\begin{array}{l}\text { Coolant water temperature at condenser } \\
\text { inlet }\end{array}$ & $75^{\circ} \mathrm{F}$ \\
\hline 26 & PNET & Net power output & $10 \mathrm{MH}$ \\
\hline 27 & WBW & Brine well flow rate & $2.5 \times 10^{6} \mathrm{LB} / \mathrm{HR}$ \\
\hline 28 & DLTPF 1 & $\begin{array}{l}\text { Pressure drop through HP leg of isobutane } \\
\text { systen }\end{array}$ & 40 psid \\
\hline 29. & DLTPF2 & $\begin{array}{l}\text { Pressure drop through LP leg of isobutane } \\
\text { system }\end{array}$ & 4.0 psid \\
\hline 30 & UPHT & $\begin{array}{l}\text { Overall heat transfer coefficient }(U) \text { for } \\
\text { HP preheater }\end{array}$ & 40 psid \\
\hline 31. & UBOILI & $U$ for HP boiler & $157 \mathrm{BTU} / \mathrm{HR}-\mathrm{ft} \mathrm{t}^{2}{ }^{\circ} \mathrm{F}$ \\
\hline 32. & UPH2 & $U$ for LP preheater & $110 \mathrm{BTU} / \mathrm{HR}-\mathrm{FT}^{2}{ }^{\circ}{ }^{\circ} \mathrm{F}$ \\
\hline 33. & UBOIL2 & $U$ for LP boiler & $145 \mathrm{BTU} / \mathrm{HR}-\mathrm{FT}^{2}{ }^{\circ}{ }^{\circ} \mathrm{F}$ \\
\hline 34. & UCOND & $U$ for condenser & 133 BTU/HR-FT2_o F \\
\hline 35. & XRAT & Flow split & 0.5 \\
\hline 36. & NEL & Electircal efficiency, & 0.95 \\
\hline 37. & NGTI & HP turbine efficiency, & 0.85 \\
\hline 38. & NGT2 & LP turbine efficiency, & 0.85 \\
\hline 39. & URG 1 & $U$ for the HP regenerator & 0.0 \\
\hline 40 & URG2 & $U$ for the LP regenerator & 0.0 \\
\hline 41 & ST1 & Dummy input & \\
\hline 42. & ST2 & Dummy input & \\
\hline 43. & DLTSHT & Degrees of superheat for HP boiler & 0.0 \\
\hline 44. & DLTSH2 & Degrees of superheat for LP boiler & 0.0 \\
\hline 45. & USHT & $U$ for HP superheater & 81 BTU/HR-ft ${ }^{2}-{ }^{0} F$ \\
\hline 46. & USH2 & U for LP superheater & 74.2 BTU/HR-FT' $-O F$ \\
\hline 47. & CPF2 & $C p \propto L P$ turbine inlet & 0.51 BTU/LB-OF \\
\hline
\end{tabular}




\section{PROGRAM LISTING \\ AND \\ SAMPLE RUN}


CCNPILER CPTIONS - NAME = MAIN,OPT=00, LINECNT $=50,5 I \angle E=000 J K$, SOURCE, EBCDIC, ACL IS T, NODECK, LCAD, MAP, NIIEDIT , NOID, XREF

ISN $0 \mathrm{CO2}$

ISN $C 003$

ISN 0004

$15 N \operatorname{CCOS}$

ISN 0006

$15 N \quad 0067$

ISN 0008

ISN OOCS

ISN OOIO

ISN 0011

ISN 0012

ISN OOI3

ISA 0014

ISA 0015

I SA COI6

ISN 0017

ISN COLS

ISN 0015

ISN 0020

ISA 0021

ISN 0022

ISN JU23 ISN OOL4

ISN CU25

ISN 0020

ISA 0027

ISA 0028

ISA 0025

ISA OU30

ISA 0031

ISN $00 \geq 2$

ISA 0033

ISA CC 34
C*** PROGRAM FOR DUAL BOILING CYCLE POWER GFNERATION SYSTEM

IMPLICIT REAL $* 3 \quad(A-F, D-Z)$

REAL $\% 8$ NGTI,NGTZ,NEL,NP,ISWT

P.EAL * 4 DEZI, GRTITL, MINL, MINT, MAXT, MEX 1, TENPLT, Z, DEUL, R.ATPLT

CEMMON/ITEF.S/ISWT (3J)

, OHCON, HSGAS, HSLIO

DIMENSICN Z2(20J), RATPLTI3U)

DIMENSION TEMPLT( 30$), G R T I T L(10)$

DIMENSITN $A(51), 6(7)$, IITLE $(20), C(51)$

DIMENSION ENTH(1O), TEMP(10), TBRINI1J),SCPPI1J), TERINS(1)

EQUIVALENCE $(A(1), T F i),(A), 2), T F 2),(A(3), P F 1),(\Delta(4), U U M M 1)$,

\& $(A(5)$, DUMM2), (A( 6$)$, DUMM3), (A) 7$), H G 1),(A(B), M G 2)$

EQUIVALENCE (A(S), TCONC), (A(1)), VFCOND), (A 111$)$, PCOND),

1 (A(12), HFCONO), (A) 13$), C P F 1),(A(14), X K 1),(A(15)$, UTHERM),

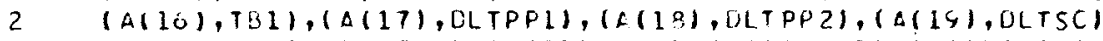

EGUIVALENCE (A(20),DTRG), (A $(21), C P B),(A(22), N P),(A(23), C P C W)$,

1 (A(24),TCWO), (A(25),TCWIN), ( $4(26), P N E T 1),(A(27)$, W'P,W),

2 (A 28$), D L P F 1),(A(25), D L P F 2)$

EQUIVALENCE (A(30), UPHI), (A (31), URCIIL), (A132), UPH2),

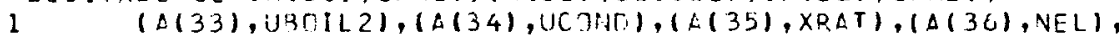

$2(\Delta(3 T), N G T),(\Delta(39), N G T 2),(\Delta(3 G), U R G 1),(\Delta(4)), U R G 2)$

$\Xi$, (A $(41)$, THLhI), (A) 42$)$, THLWD),

E(A $(43)$, DLTSHL), (A (44), CLTSH 2$),(A(45)$, USHI

4), ( $14(4 t)$, USH2) , (A (47) , CPF 2$),(A(48), C$ CF 3$),(A(45), C P F 4),(A(5)), C P F 510 B V 20330$

EQUIVALENCE IA(SI), RATIOB)

UATA IPLT/JI

DATA CKATIC,URATIOI.1,.01/

CLLL FCHL

IShI(I) $=$ DUN, MI

I SWT $(2)=$ DIJMMI 2

$\operatorname{IS} \operatorname{Sin}(3)=$ DUJNM 3

$X K=X K 1$

C REAO IN VAFíUUS CATA FOR TABLES

4 READ $(5,16 J 2)$ NSUBSC, DELT

1 GO2 FORMAT $(12, F E .2)$

FEE AD (5,1603) GRTIIL

1EJ3 FORMAT(1OA4)

NPOINT $=0$

$C$ C* KEAC IITLE ANL PRIMARY INPUT DATA

DRV 20340

DPV 20350

DBV 20360

$D B \vee 2037 \mathrm{~J}$

DRV 20380

DRV 20390

DBV 20400

CRV 20410

ORV 20420

$O B \vee 20430$

D) 9 V 20440

DBV 20450

$03 \vee 20460$

$03 \times 20470$

DRV 23430

DRV 20490

$C$

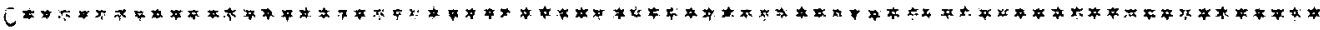

C

C

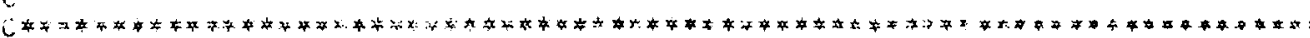

5 KEADI5, $959 I T I T L E$

I C T $3=$ J

ICT $T_{4}=0$

ICT $5=0$

ICTO $=0$

$\angle C P C=0$

SSG F(RMAT $(2014)$
DBV2Jis10

DRV 20520

$03 \vee 20530$

DAV 20550

URV 20560

DHV 20570

DRV 2059J

DQV 20590

DRV 20600

DQV 23010

DAV 20620 
ISN 0035

I $S \mathrm{~N} 0036$

ISN $\quad 0037$

$15 A \quad 0 C 35$

ISN CC4I

ISN 0042

ISN 0043

ISN 0044

ISN 0045

ISN 0046

ISN 0047

ISA OC48

ISN 0049

ISN OC5O

ISN COE 2

ISN 0053

I SN CO55

ISN OOEE

ISN 0057

ISN 0058

ISN 0055

ISA CO6O

ISN OCGI

ISN 0022

ISN OCE 3

ISN CUE 4

ISN 0065

ISN 0067

ISA 0068

ISA CC69

ISA COTO

ISN 0071

ISN OC73

ISN OC74

ISN 0075

I SN CC76

ISN 0077

ISN 0078

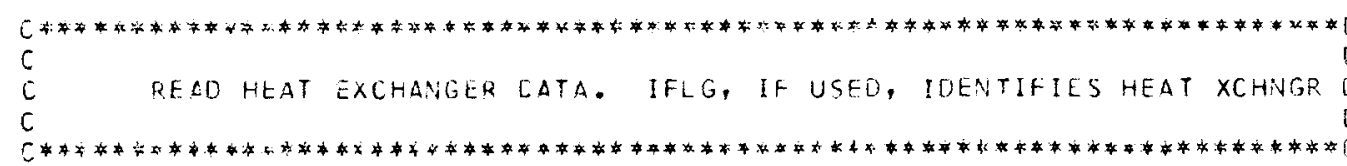

REALI 5,130$) A H I X, I F L G$

$1 \equiv 0$ FCRMAT $(1 X, E 10.4, I 5)$

IF (IFLG.E.G. U) IFLG=1

IF(IFLG .NE. 10) GO TO 100

READ(5,131) ABOL1, AEOL2, 4 TH1I, ATH2I

131 FORMAT $(1 X .4 F 10.3)$

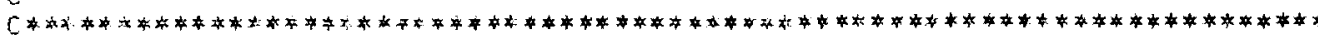

C

c

CATA SET CARD WITR NSTCP CONTROL

100 REAO(S,S8S) SSTCP, NPLTSW, NCASE, INDEX1, INUEX2, (B) I I, I =1, INDEXZ)

S39 FORMAT $1311,12,1 X, 12,2 X, 7 E 10.41$

$I I=1$

NALL = INUEXI+INOEX2-1

DT: 112 I = INDEXI, NALL

$\dot{A}(I)=Q(I I)$

$112 I I=I I+1$

IFINSTOP.GE, 1 I GO TO 6

GOTO 100

$C$

$c$

C HAVE CCMPLETED CASE INPUT CATA READ

6 IFINSTOP .NE. 21 GO TO 8

(j) $7 \quad I=1,50$

$C(I)=A(I)$

7 EENTINUE

$8[C K]=J$

$I C K 2=J$

RRAT $=$ XRAT

LNIJM $=18$

$\angle R A T=-.2$

$X P I N 1=$ DLTPPI

$X P I N Z 2=D L T P P 2$

IF INSTOP .NE.4 .AND. NSTOP. .NE. 6 I GO TC 9

CALL PROPH(PPP,TF1,5)

ENTGGI = HSGAS

11 CALL PROPH(PF1, ENTGG1, 1)

CALL ITER (3, ENTGC1, TEMPI, TFI, FLAG)

IF (FLAG.NE. 0.0$)$ GO TO 11

$H I 1=E N T G G 1$

CALL PROPH(PCONU, TCCND,5)

CI]NDH = HSGAS

$H F G I=H I I-H S L I O$

HFCOND = DHCCN

$T I 1=T F I$

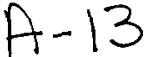

DRV20640

$08 \vee 20670$

$03 \vee 20680$

$03 \vee 20650$

DRV $207 J 0$

DAV 20710

DBV 20720

Dค $\vee 20730$

DQV20740

OBV 20750

DBV 20770

DBV 20780

DSV $2 J 700$

DRV 20800

DRV 20820

DeV 20830

DRV 20840

DAV 20350

DBV 20860

DBV 20870

DBV 20380

$D B \vee 20890$

DRV 20900

0 $08 \vee 20910$

DBV 20930

* $08 \vee 20043$

DRV 20960

DBV 20970

DBV 20980

D $\vee 20990$

DBV 21000

DBY 21010

DBV21J20

อBV 21050 DBV 21060

DBV 21070

DBV 21080

DAV 21090

DRV 21100

DBV21110

DQV 21120

DBV 21130

DBV 21140

D९V 21150

DBV21160

DQV 21170

DBV 21180 


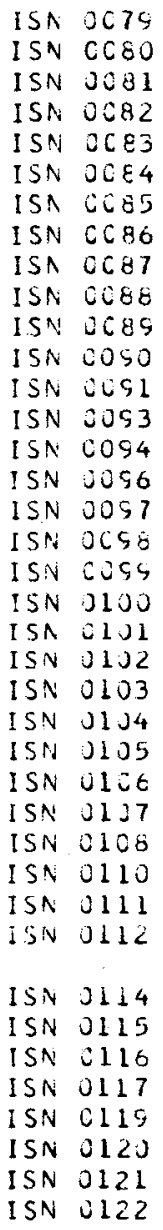

ISN $01 \angle 3$ ISN 0124

ISN 0125

ISN 0127

ISN 0129

ISN 0129

I $5 N \quad 0130$

ISN 0131

ISN OII32

ISN OI33
LNUN: $=1$

GO TO 12

\& CONTINUE

CALL PROPHIPFL,TF1,SI

$H G I=H S G A S$

$F F G I=D H C O N$

CALL PQOPH(PF L, TFZ, 5$)$

$H G Z=H S G A S$

$H F G 2=D H C D N$

(ALL PKCPH (PCENO,TCCNO,5)

CONCH $=H S G$ IS

HFCOND = DHCCN

IF (RRAT. NE. 1JJ.) LNUM = 1

DO $4235 \mathrm{~L}=1$. LNUM

IF IP.HAT.NE. 100.) GO TO 12

$\angle R A T=\angle R \Delta T+.1$

$X R A T=\angle R A T$

i2 PGKRSS = PNETI

$D L T P P I=X P[\Lambda]$

$D L T P P 2=X P I N 2$

WRITE $(6,800)$ TITLE

$80 J$ FURMAT( $1 H 1.2044)$

WRITE $(6,802)$

BU2 FCRMATUIHU,OJX, 'INPUT EOIT THIS CASE'I WRITE $(0.700)(A(I) .1=1,50)$

700 FSQMAT (IHO, IPLEE13.4)

WRITE (S, 13 UIGHTX, IFLG

IFIIFLG. .EQ. 1U) WRITE (C, I31 IABOLI, ABOL2, ATHII, ATH2I

DTRG2 = DTKG

DTRGI = DTRG

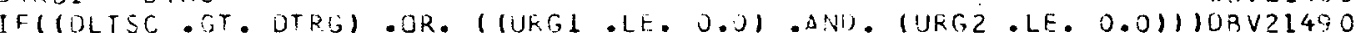

IUTRG $=D L T S C$

$\triangle P F=(C P F 1+C P F 2) / 2$.

$I C T M=0$

$A R r_{3}=J . J$

IF (NSIMP .EQ. 4 . OR. NSICP.EQ. 0) OJ TC 41

TIL $=$ TFI+DLTSHI

$H 11=H S_{1} 1+C P F 1 * D L T S H 1$

TI $2=T F Z+O L T S H 2$

$H 12=H G 2+C P F 2 * D L T S H Z$

$c$

C \#\# EOMPUTE BRINE SUPFRHEATER ONE DUTLET

$\angle \angle S=C P F 1 \% D L T S H 1 / H F G 1$

$T \triangle 1 P=(T B 1+2 \angle 5)(T F L+C L T P P 1) /(11 .+2 Z 5)$

10 IF XXFAT.LT. J.J GO TO 40

$I C K I=0$

$I C K 2=J$

C** LSSLME PINCH PCIAT CAE CINTROLS

$T B L P=(T E L+Z Z S *(T F 1+C L T P P()) / 11 .+2 L 5)$

$T B 3=T F 1+D L T F P 1-C P F 5 / F F C_{3}+(T F 1-T F 2) \div(T B L P-T F 1-D L T P P I)$

$C$ \# CCMPUTH PINCH PLINTT TWC

$Z Z O=H+G Z / H F G 1 * X R A T$

$\angle 27=C$. PF $2 * D L T S H 2 / H F G 1 * X R A T$

$D L T P P 4=T B 3-(\angle \angle 6+\angle Z T) *(T B L P-T F 1-O L T P P 1)-T F 2$
DBV 21190

DBV 21200

$D B \vee 21210$

DBV21220

DBV 21230

DAV 21240

DQV21.250

DRV 21260

DRV 21270

DBV 21280

DBV21250

OBV 21300

DRV 21310

DEV 21320

DBV 21330

DBV 21340

DBV 21350

$D Q \vee 21360$

DBV 21370

DBV 21390

DgV 2141$) 0$

DBV 21410

DBV 21420

DRV 21430

DRV21440

DBV 21450

DSV 21460

OBV 21470

DRV 21480

UBV 21500

DRV 21510

DBV 21520

OBV 21530

D. 211540

DBV 21550

DBV 21560

DRV21570

DRV 21580

RTDBV 21590

DBV216)

DHV 21610

OBV 21620

DBV 21630

DBV21640

DBV 21650

$08 \vee 21660$

URV 21670

DBV 21680

DBV21690

DBV 21700

D $3 \vee 21710$

OQV 21720

DRV 21730

Dev21740

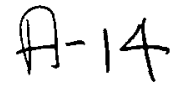




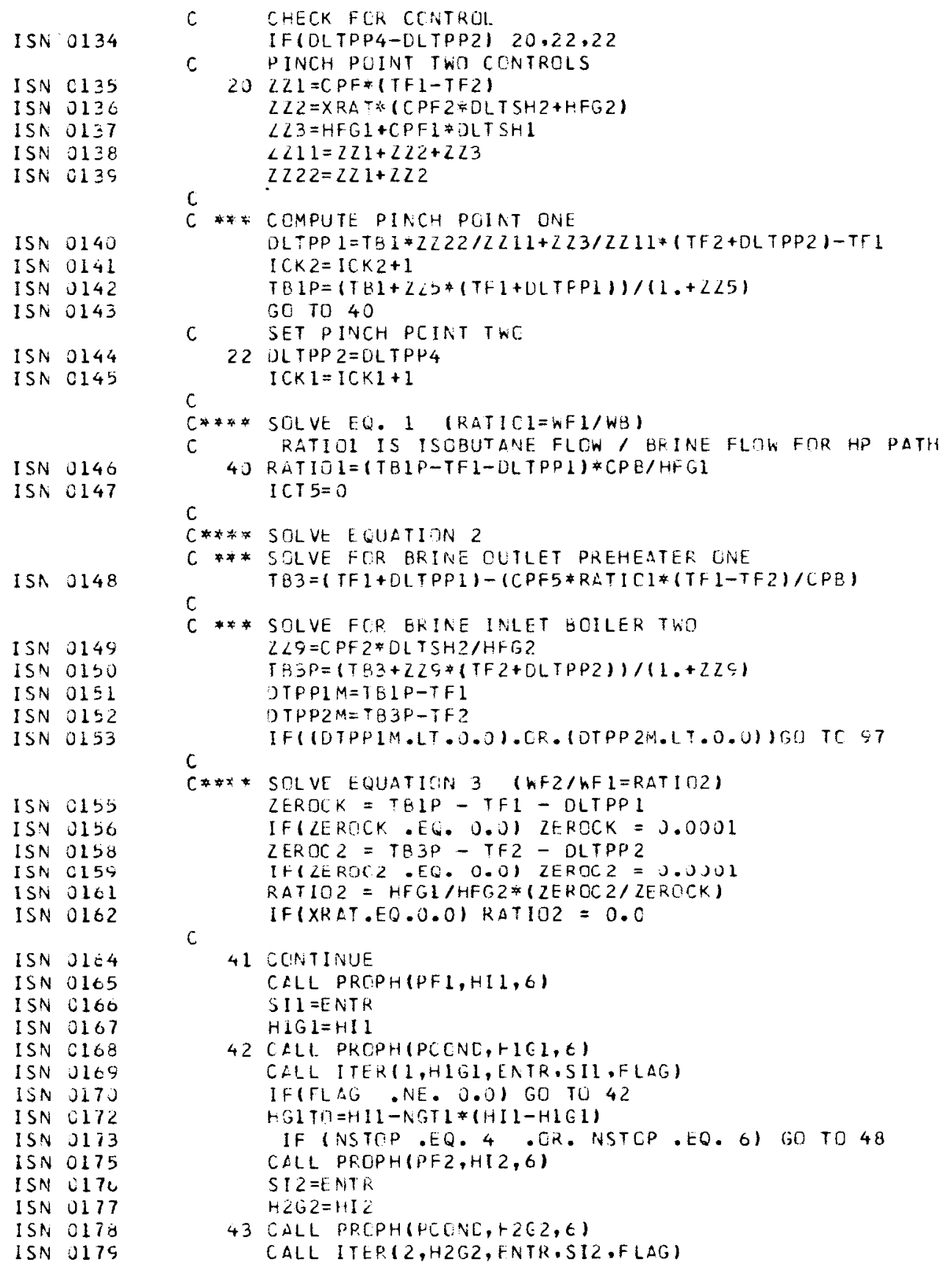

DBV 21750

$D B \vee 21760$

DSV 21770

DBV 21780

OBV $217 \subseteq 0$

DBV 21800

DBV 21810

DRV 21820

DBV 21830

UBV 21840

DRV 21850

CB V 21860

DRV 21870

DBV 21880

DBV 21890

DBV 21900

D $V 21910$

DBV 219? 0

DQV 21930

DBV21940

$D B \vee 21950$

$D B V 21460$

[OBV 21970

DBV 21980

DBV 21590

$D B \vee 22000$

DBV 22010

DRV 22020

D $3 V 22030$

DRV 22040

DBV 22350

DBV2?060

DBV 22070

DRV 22080

D 8 V22100

D $V 22110$

DQV 22120

DRV 22130

D $\vee 22140$

DBV 22150

DBV $2 ? 160$

DBV 22170

DRV 22180

$08 \vee 22190$

$D B \vee 22200$

DRV 22210

DBV 22220

DBV 22230

DBV 22240

DBV 22250 
ISN 018 I J
ISN 0182
ISN 0183
ISN 0184

ISN 0185

ISN 0186
ISN 0187
ISN 0189
ISN 0191
ISN 0192
ISN 0193
ISN 0194
ISN 0195
ISN 0156

ISN CIST

ISN 0158

ISN 0200

ISN 0201

ISN 0202

ISN 0203

ISN 0205

ISN 0207

ISN 3208

ISN 0205

ISN 0210

ISN 0211

ISN 0212

ISN 0213

ISN 0214

ISN 0215

ISN 0217

ISN JE 18

ISN 0218

ISN $022 \mathrm{~J}$

ISN U221

ISN 0222

ISN 0223

ISN 0224
IFIFLAG -NE. 0.01 GO TO 43

HGLTO=HI2-NGT2*(HI2-H2G2)

C* * SOLVE EQUATION 7 FOR QTI

48 CONTINUE

QT $=N E L *(H I 1-H G L T \subseteq)$

$C$

C* * SCLVE ECUATICN 8 FOR QPWL QPW $1=0.185 * V F C O N D *(P F 1-P C O N O+O L P F 1) / N P$

C*\#\# SOLVE EQUATION S FOR QCWL

DELHR I = HG ITC-CONDH-CPF 3*IDTRG-DLTSC)

IFIUELHRI -LE. J.J) GC TO 44

IFI UKGI.LE. 0.0$)$ GO TO 44

$Q C W 1=0.263 *(H F C C N D+C P F 3 * D T R G) /(C P C W *(T C W D-T C W I N))$

DELR $1=D L L H R 1$

GO $10 \quad 45$

$44 Q C W 1=0.263 *(D E L H R I+H F C O N D+C P F 4 * D L T S C) /(C P C W *(T C W D-T C N I N))$ DTRGI $=0$ LTSC

$D E L R I=0.0$

$c$

C*** SOLVE EQUATICN IO HCR GNETI

45 UNETI $=Q T 1-C P W I-G C W I$

IF (NSTOP .EQ. 4 .OR. NSTOP. EQ. 6) GO TO 49

$c$

C*** SCLVE EQUATICA 11 FLR QT2

$Q T 2=N E L \#(H I 2-H G 2 T[1)$

$c$

C*** SOLVE EQUATION 12 FCR GPW2

QPW $2=0.185 * V F C O N D *(P F Z-P C O N D+D L P F 2) / N P$

$c$

C* $*$ SULVE FQUATICN 13 FCR GCW2

DEL. HK $2=H G 2 T O-C O N O H-C P F 3 *$ (DTRG-DLTSC)

IFIDELHR2 -LE. U.J) GC TO 46

IFIURG2 .LE. 0.0$)$ GO TO 46

QCW2 $=U .263 *(H F C C N D+C P F 3 * 0 T K(S) /(C P C W *(T C W C-T C W I N))$

LELR2 $=1$ ) ELHFi 2

GC $10 \quad 47$

$462 C$ W2 $=0.263 *(D E L H K 2+H F C O N D+C P F 4 * D L T S C) /(C P C W *(T C W D-T C W[N))$ DTROL $=D L T S C$

$D E L R 2=0.0$

$c$

C*:* SOLVE EQUATICN I\% FCR GNET2

47 QNET2=QT2-QPWZ-QCW2

49 CONTINUE

IF INSTOP . NE. 4 .AND. NSTOP. NE. 61 GOTC 70

HGLTD $=0$.

QT2 = 0 .

GNET $2=0$

GPW2 $=0$.

QCW2 $=0$.

$c$

C \# FINU TENPERATURES TF THE FLUID IN THE HEATEF DELEN = (HFCL - DELRL)/10.

ENTH(i) $=H I 1$

JC $50 \quad K=2,10$
DBV 22260

DBV 22270

DBV 22280

OBV 22290

DRV 22300

DBV 22310

DBV 22320

DBV 22330

DBV 22340

DRV 22350

DBV 22360

DBV 22370

DBV 22380

DBV22.390

DBV 22400

DRV 22410

DBV 22420

DRV 22430

DBV 22440

DPV 22450

DBV 22460

DBV 22470

DRV 22480

DBV 22490

DBV 22500

DBV 22510

DRV 22520

DBV 22530

DBV 22540

DRV 22550

DBV 22560

DRV 22570

DBV 22580

DRV 22590

DBV 22600

OBV 22610

DBV 22620

DBV 22630

D) BV22640

DRV 22650

ORV 22660

DBV 22670

D 8 V 22680

$0 B V 22600$

URV 22700

DBV 22710

DBV 22720

DBV 22730

$09 \vee 22740$

UR 22750

DBV 22760

DBV22770

DRV 22780

DBV 22790

DRV 228J0

DBV 22810

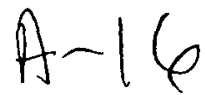




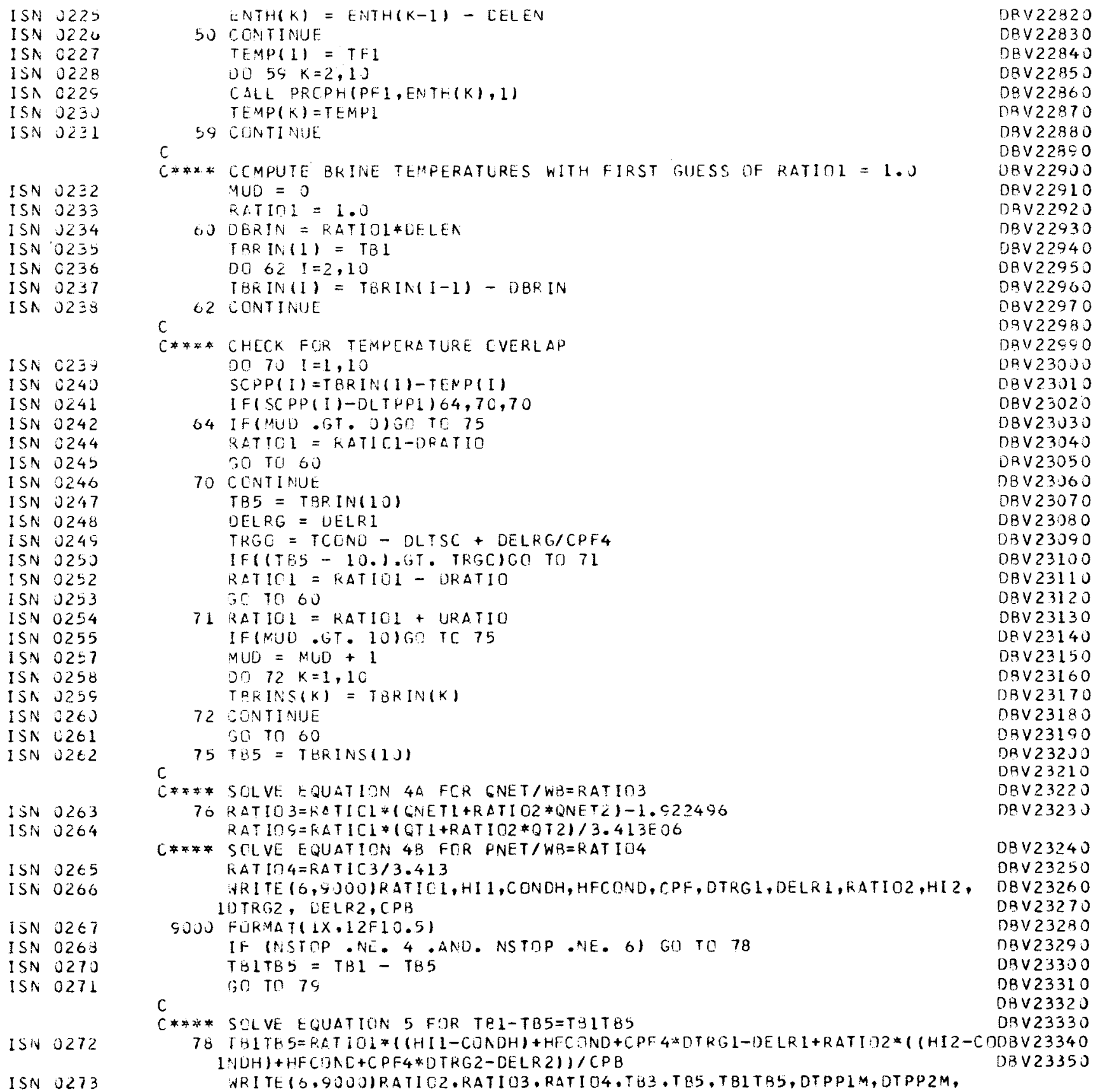

ISIN 0272

ISN 0273

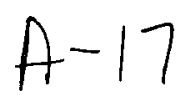


$c$

LITTPPIN.DTPP2M

ISN 0274

C** SOLVE EqUATION Ó FOR EFF

$79 E F F=(R A T I O 3 /(C P B * T B I T B 5)) * 100.0$

C

ISN 0275

ISN 0276

ISN 0277

ISN 0278

ISN 0279

ISN 0280

ISN 0281

ISN 0282

ISN 0283

ISA $C 284$

ISN 0283

ISN 0286

ISN $C 280$

ISN $02 \mathrm{EC}$

ISN 0250

ISN U2S 1

ISA 0292

ISA 0253

ISN $02 \mathrm{CS}_{4}$

ISN 0255

ISN 0256

ISN 0257

ISN C258

ISA U2S9

ISN J 300

ISA $03 \mathrm{CI}$

I SN 0303

ISN 0304

ISN 0300

ISN 0306

ISN 0307

ISA 0308

ISN 0310

ISA $0 \equiv 11$

ISN 0312

ISN 0313

ISN OEIS

ISN $0 \equiv 10$

I SN $0 \geq 17$

ISN J 218

ISN $0 \equiv 19$

$15 N 0320$

ISN 0321

ISN 0322

ISN 0324

ISN 0325

C** SCLVE EQUATICN IS THRU 23

INE=PGROSS/RATIOS

$W F 1=R A T 101 * W B$

WF $2=F A T 102 *$ hH 1

$Q B P=1.922496 * W B / 3.413 E 06$

$Q C W=(W F) * Q C W 1+W F 2 * 6 C W 2) / 3.413 E) 6$

$Q F P=($ WF 1 *QPhI WWF 2 Q $Q P W 2) / 3.413 E 06$

NOWW $=W B / 7.51 E O 5+C .99$

NCB WK $=N$ NGW/2

PNET $=$ KATID3*hB/3.413EC6

PNTPGR=PNET/PGROSS

$P N P W E L=P N E T * W E W / W B$

IF (NSTOP. .NE. 4.AND. NSTOP .NE. O) GO TC 87

$\triangle P H I=0.0$

$A B 2=0.0$

$A P H 2=0.0$

$\triangle S H I=0.0$

$\triangle S H 2=0.0$

$A S H=0.0$

$\Delta 2=0.0$

DTEAT $=W B$ T31THS

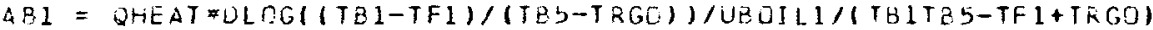

$A 1=A B 1$

A SUM $=21$

G. TO 55

87 AEL =W 4 *CPE DLOG( TELP-TFI)/CLTPPL)/UBCILI

I $F((T F 1-T F 2)$. LE. U.0) GO TO 80

$\triangle P H 1=W B *(P B * D L O G((T B 3-T F Z) / O L T P P 1) /(U P H 1 *(1 T F 1-T F 2) /(T F 1+D L T P P 1-$

1

GO TO $E$ $(B 3)-1.0)\}$

$\& 8$ A PHI $=0.0$

$8 S \quad \triangle 1=\Delta t 1+\Delta P+1$

C**** SOLVE EQUATICNS Z4THRU 32

$\triangle B 2=W B(P B D L C G(1 T B 3 P-T F 2) / D L T P P 2) / U B C I L 2$

IF(OL TSHI .LE. 0.0) GO TO 90

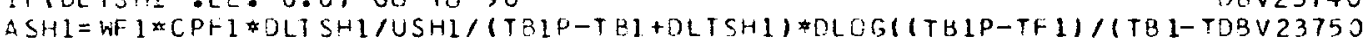

II 111 GE TO $S 1$

90 \& $S H 1=0.0$

S1 IFIDLISHZ .LE. 0.01 GO TO 92

DBV 23360

DRV 23370

DBV 23380

$09 \vee 23390$

DB $234 J 0$

$D B \vee 23420$

DBV 23430

DBV 23440

DBV 23450

DBV 23460

D) 823470

DBV 23480

DBV 23500

DBV 23510

OHV 23520

DBV 23530

DQV 23540

D) B 23550

$08 \vee 23560$

DAV 23570

DBV $235 R \mathrm{~J}$

DBV 23590

ORV 23600

DBV 23610

DBV 23620

OBV 23630

D $8 \vee 2364 \mathrm{~J}$

DBV 23650

DRV 23660

$O B \vee 23670$

DBV $236 \geq 0$

DAV 23690

DQV 23700

DP. 23710

D $8 \vee 23720$

DBV 23730

DEV23740

DBV 23760

DBV $2377 \mathrm{~J}$

DRV 23780

DRV 23700

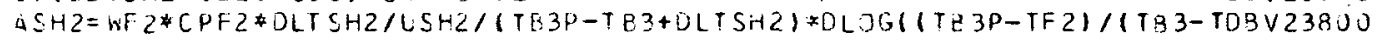

(I 2) ।

5C TO $5 \mathrm{a}$

$92+\mathrm{SH2}=\mathrm{J} . J$

53 A $S H=A S H 1+\Delta S H 2$

$D E L R G=(O F L R I+R A T I \Gamma 2 *(D E L P, 2)) /(1,+R \Delta I I \cap 2)$

TFC, $=T C O N D-D L T S C+L E L R G / C P F 4$

$T S z=-(T B 1 T B 5-T E 1)$

IF( (TB5-10.) . GT. TFEC) 60 TO 94

DQV 23810

DRV 23320

DBV 23830

OBV 23840

D $8 \vee 23350$

D $9 \vee 23850$

DBV 23870

DBV2389J

57 CONTINUE

DTPPIN:=DLTPFI+([APS(DTPPIM-DLTPPI)/2.)

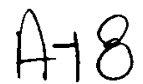




\footnotetext{
$15 \mathrm{~N} 0326$

ISN 0327

ISN 0329

ISN 0331

I SN 0333

ISN 0334

I $5 N \quad 0335$

ISN 0336

ISN 0330

ISN 0339

ISN 0341

I $5 \mathrm{~N} 0342$

ISN 0343

ISN 0344

ISN 0342

ISN 0346

ISN 0347

ISN 0348

ISN 0349

ISN 0351

I SN $03 E 2$

ISN 0354

ISA 0356

ISN 0357

ISA 0358

ISN 0359

ISN 0360

ISN 0361

ISN 0362

ISN 0363

ISN 0365

ISN 0367

ISN 0308

ISN 0370

ISN 0371

ISN 0372

ISN 0373

ISN 0374
}

ISN 0375

ISN 0376

ISN 0377

ISN 0378

ISN C379

ISN $038 \mathrm{~J}$

ISN C381

ISA 0382

I SN 0383

ISN 0384

I SN 0385

ISN 0386

ISN 0387
DTPP2MI=ULTPP $2+(C A B S(O T P P 2 M-C L T P P 2) / 2.1$

IF( (ICK1.GE.1) .OK. (XRAT .LT.0.0) )ULTPPI=DTPPIN

IF( (ICKZ.GE.1) .OR. (XRAT.LT.J.OU) ULTPPZ =DTPP $2 N$

[FIICTM . GT. IOI GO TO 94

DBV 23930

ICTM=ICTM+1

SC T? 1 I)

$D B \vee 23940$

DRV 23950

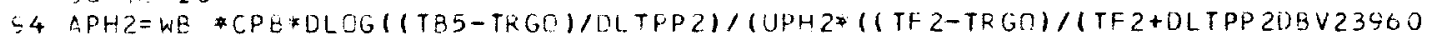

$1-T B 5)-1)$

IF(I ICTM . GE. 10) .ANC. (ICKI .GE. I) VIRITE $(6,2130)$

2130 FGRMATILX, TEMP ECILER JNE IS TOQ SMALL ')

IF(IICTN .GE. 1O) . ANO. (ICK2 . GE. II) WRITE(6, 2140)

2140 FoRMATIIX, TEMP BCILER TWE IS TCO SNALL 1

$A 2=A B 2+A P H 2$

$\triangle S U M=A L+A 2$

S5 CONTINUE

NF $11=$ hF 1

$W F 22=W F 2$

$\triangle T H E R M=0.0$

1.) THEXV=0.0

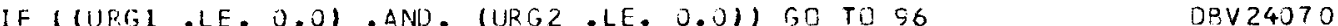

$\triangle R G=W F 1 * D E L R 1+W F 2 * D E L R 2$

IF(URGI . LE. 0.0$) \quad W F 11=0.0$

IF(URGZ . LE. 0.0$) \quad W F 22=0.0$

So $H M I X=(W F 11 \pm F-T T O+W F 22 * G 2 T O) /(W F 11+W F 22)$

$T M I X=H M I X / .7250+1007$.

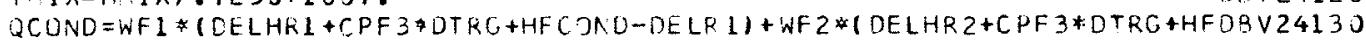
1CQND-DE LR2 I

TFCO $=T C O N D-D L T S C$

CONDTO=TFCT-TCWIN

CCNOTI $=$ TMIX-TCWO

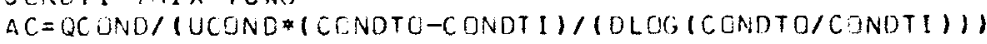

IF(IURGI :LE. J.0) . AND. (URG2 .LF. 0.0)) GO TO 98

IF (TMIX-TRGO) .LE. (.5*DTRG) TMIX TRGO+.5*DTRG

DBV 23980

LRV 23990

DRV 24000

DEV 24010

D $B \vee 24023$

DBV 24030

OgV 24040

DBV 24050

DBV 24060

DRV 24080

$D Q V 24090$

DRV 24100

DPV 24110

DEV 24140

DRV 24150

DEV 24160

DBV 24170

DeV 24180

DaV 24190

DBV 24200

$A R G=Q R G /((U R G 1+U K G 2) / 2$. $(D T R G+D L T S C-T M I X+T R G O) / D L O G($ (DTRG +DLTSC)/(DBV24210

ITMIX-TRGOI))

IF((URGI . LE. 0.0) .OR. (URG2 .LE. O.01) $\triangle R G=\angle R G \# 2$.

58 HTA $=\Delta 1+A 2+A C+A R G+\triangle S H$

$W C W=Q C O N D /(C P C h *(T C H C-T C W I N))$

$G P M=N C W / 500.0$

$N G C W=h C W / 7.51 E O b+.99$

NOC $W R=N O C W / 2$

C

OVERALL EFF OF ENERGY SUPPLIED

QALN=RATIOS/CPB/ TBRI-TCWIN)*100

GO TD $(129,120,121,122,123,124,125,126,127,129)$, IF́LG

$120 \quad A C K=H T A$

GO TO 128

$121 A C K=A S U M+A S H$

SO TO 128

$122 A C K=A B 1$

SO TO 128

$123 \mathrm{ACK}=A B 2$

G0 10128

$124 \mathrm{ACK}=\mathrm{APH} 1$

GO TO 128

$125 \mathrm{ACK}=\mathrm{APH2}$
D 924220

D) $P V 24230$

UAV 24240

D\&V 24250

D B 24260

DBV 24270

DBV 24280

[) $B \vee 24290$

$D B \vee 243 J 0$

DBV 24310

D $8 V 24320$

D $8 V 24330$

DAV 24340

DBV 24350

DRV 24360

DBV 24370

DAV 24380

DBV 24390

DBV $244 J 0$

DBV 24410

DBV 24420

DBV 24430 


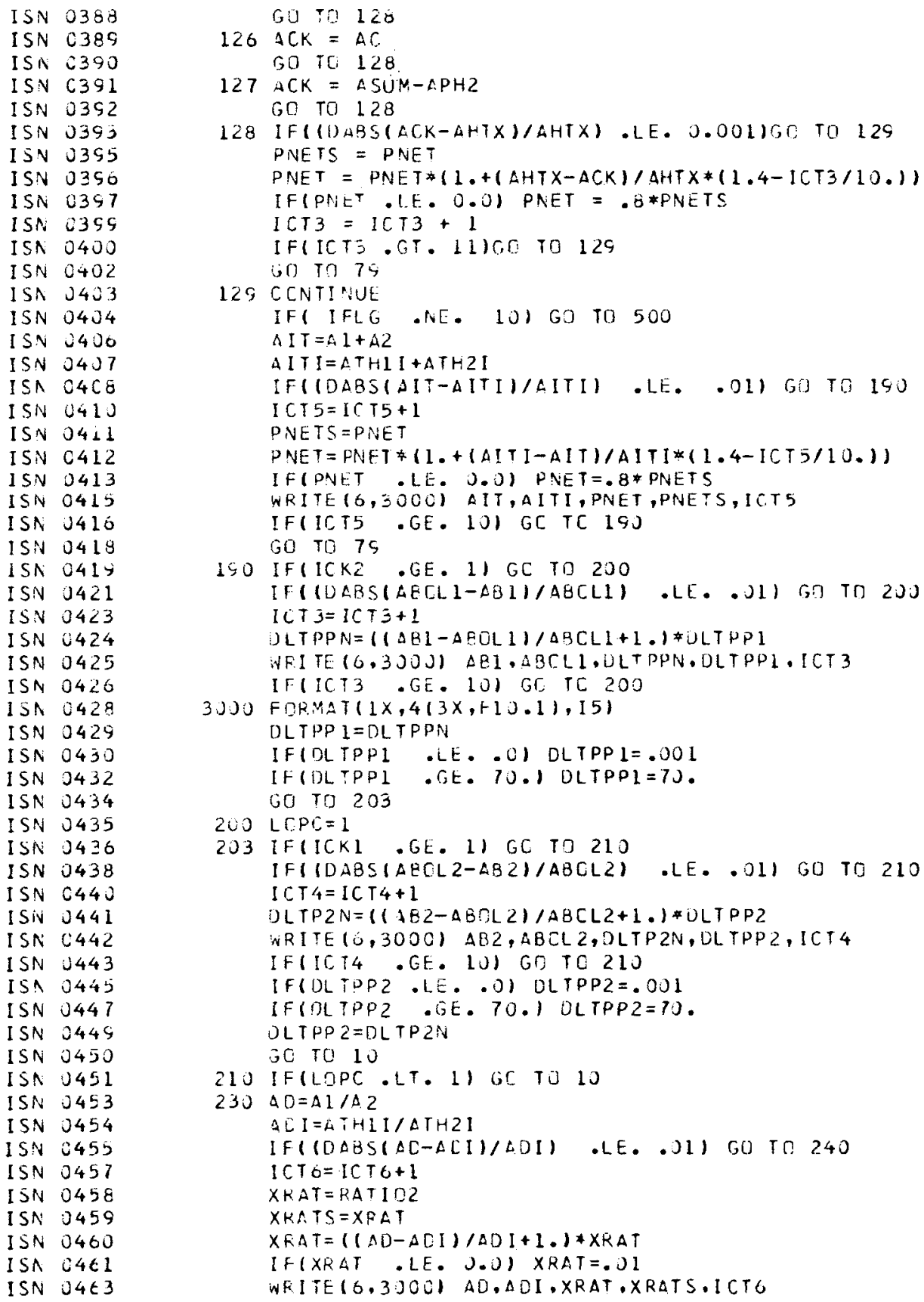

DBV 24440

DBV 24450

DAV 24460

DAV 24480

DAV 24490

DRV 24500

DAV 24510

DBV 24520

DSV $2453 \mathrm{~J}$

DBV 24540

DBV 24550

DBV 24560

DBV 24570

D $\vee 24580$

DBV 24390

DBV246J0

D $8 V 24610$

$D B \vee 24620$

DBV 24630

DRV 24640

DBV 24650

DBV 24660

DBV 24670

DBV 24680

DBV 24690

DAV 24700

DBV 24710

D 824720

DBV 24730

UBV 24740

DBV 24750

OBV 24760

DBV 24770

DBV 24780

DBV 24790

DAV24800

DBV 24810

DRV 24820

DBV 24830

DBV 24840

$D B \vee 24950$

DBV 24260

DBV 24870

DRV 24880

UBV $248 \subseteq 0$

DBV 24900

DR 24910

$D R \vee 2492 \mathrm{~J}$

DBV 24930

DRV 24940

DBV 24950

DEV 24960

DBV 24970

DBV 24980

DBV 24990

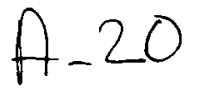


ISN 3464

ISN 0406

ISN 0468

ISN 0465

ISN 0470

ISN 0471

ISA 0472

ISN $C 473$

ISN 0474

ISN 0475

ISN 0476

ISN 0477

ISA 0478

ISN 0475

ISN 0480

IS:I C48I

ISN 3482

ISN 0483

ISN $J 4 E 4$

ISN 0485

ISA 0486

I SN 0487

ISN 0488

ISN 0489

ISN J490

ISN 0451

ISN 0492

ISN 0453

ISN 0454

ISN 0455

I Siv 0496

ISN 0497

ISN 0498

ISN 0499

ISN 0500

ISN 0501

ISA 0502

ISN 0503

ISA 0504

ISN C5OS

ISN 0506

ISN 0507

ISA 0508

ISN O5OS

I $5 \mathrm{~N} 0510$

I SN 0511

ISN OEI2

ISN 0513

ISN $0 \leq 14$

ISN 0515

ISN UE 16

ISN 0517

ISA 0518

ISN USIS

ISN $0 \leq 20$

ISN OS 21
I FI XFAT .GE 4 . I) XRAT $=4$.

IFIICTS .EE. 10 GC TC 240

ICT $3=0$

ICT $4=0$

GC TO 10

240 CONTINUE

500 NRITE $(6,803)$

RATIOE = KATIC2

EO3 FORMAT (LHU.1OX. 'CASE RESULTS')

WRITE $(6,850)$ RATIOZ

85J FJRMAT(IHJ, 'QNET/WB (BTU/LB)', IPE1 J.4, $5 \mathrm{X}$, 'EQUATION 4A') WR ITE $(6.851)$ RATIO4

651 FORMAT(1HO, 'PNET/WE (WATT-HR/LE)', IPE 12.4,5X, 'EQUATION 4B') WRITE $(6,852)$ TEITB 5

852 FORMAT(IHO, DELTA T BRINE (DEGREES F) , 1PE12.4,5X, 'EQUATION 5') WRITE $(6,853)$ EFF

E53 FCRMATIIHO. 'THERMAL EFFICIENCY NET (名)',IPE12.4,5X.'EQUATION 6') WRITE $(6,854)$ PNP'NEL

854 FORMAT(1HO, "PNET/WELL (LB/HR)', 1PE12.4,5X, 'EQUATION 20') WRITE $(6,855)$ RATIOI

GS5 FORMATIIHO, 'WF1/WE', IPE $12.4,5 X$, 'EQUATIIJN 1 ') WRITE $(6,856)$ RATIT2

855 FORMLT(IHO, 'WF2/WF1,,1PE12.4,5X, 'EQUATICN 3') WRITE $(6,857)$ TB3

ES7 FCRMLT(IHJ,'TE3 (CEGREES F)', IPE12.4,5X. 'EQUATION 2 ') VRITE $16,85 E) Q T 1$

858 FORMATIIHO,'QTI (BTU/HR)', IPE12.4,5X, 'EQUATION 7') WRITE $(6.855)$ QW 1

85 S FCRMAT(1HO, 'QPW1 (BTU/LB)', IPE 12.4,5X, 'EQLATION 8 ') WRITE $(6,860)$ UCW 1

860 FOKMEIIIHO, 'GCWI (BTU/LB)', IPE 12.4,5X,'EQUATIDH G') WRITE (6, J6I) ONETI

E6I FCRMATIIHO, 'QNETI (BTL/LE)".1PE12.4.5X, 'EGUATION 10") WRITE $(6,862) 0 T 2$

862 FOKMAT(1HO, 'QT2',1PE12.4,5X, 'EQUATION 11') WF. ITE $(6,863) 6$ PW2

EG3 FIRMATI1HO, 'PW2', 1PE12.4,5X, 'EQUATION 121 WRI TE $(6,864) 0 \mathrm{CW} 2$

864 FIMRATI IHO, 'QCW2', 1PE12.4,5X,'EQUATIOA $13 \cdot 1$ WRITE $(6,865)$ QNET2

E65 FORMAT(1HO, 'GNET2 (BTU/LB)',1PE12.4.5X.' EGUATION 14'1 WRITE $(6,866)$ PNET

8U6 FORMAT (1HO, 'PNET (NEGAKATTS)", IPE12.4,5X, 'INPUT') WRITE $(6.867)$ PGRESS

O67 FORMATILHO, 'PGROSS (MEGAWATTSI', IPE12.4,5X, 'EQUJAION 18') WKITE $(0,868)$ PNTPGR

868 FORMAT( 1 HO, 'PNET/PGROSS , 1PEI2.4,5X, EQUATICN 19') WRITE $(6,869)$ WE

869 FCKMAT(1H).'WE (LE/FR)',1PE12.4.5X.'EQUATICN 16') WRITE $(6,870)$ WF 2

87U FURMAT(1HO, 'WF2 (LB/HR)', IPE12.4,5X, 'EQUATION 17') WRITE(6.871)TFI

871 FORMATIIHO, 'TFI (OEGREES FI', IPE12.4,5X,'INPUT') WRITE $(6,872)$ TF2

872 FORMATI IHO, 'TF2 (DEGREES F)', IPE 12.4,5X, INPUT') WKITE $(6,873) \triangle B 1$
DBV 25000

DBV 25010

$D B \vee 25020$

$D B \vee 25030$

$D B \vee 25040$

$D B \vee 25050$

DRV 25060

$D B \vee 25070$

DBV 25080

DBV 25090

DRV 25100

DBV 25110

DBV 25120

DBV 25130

OBV 25140

UBV 25150

DRV25160

D $8 \vee 25170$

DBV25180

DBV $251 \subseteq 0$

DBV252J

DBV 25210

DBV 25220

1) BV2523 J

DAV 25240

DBV 25250

$D B \vee 25260$

DBV 25270

DBV2528J

DBV 25290

DBV 25300

DBV 25310

OBV 25320

DBV 25330

DAV 25340

DBV 25350

DBV 25360

DBV 25370

DEV 25380

DBV 25390

DBV 25400

DBV 25410

DRV2542 D

DSV 25430

DBV 25440

DEV 25450

DBV 25460

DBV 25470

DeV25480

DRV 25490

DRV 25500

DBV 25510

$D B \vee 25520$

DBV 25530

ก 8 V 25540

$D B \vee 25550$

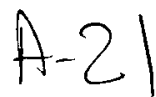


ISN 0522

ISN OE23

ISN $0 \leq 24$

ISN 0525

ISN $0520^{\circ}$

ISN $0 \leq 27$

ISN J528

ISN 0529

ISN 0530

ISN $0 \leq 31$

ISN 0.32

ISN 0533

ISIN $0 \leq 34$

ISN 0535

ISN UE 36

ISN 0537

ISN 0538

ISN 0539

ISN 0540

ISN 0541

ISN 0542

ISN 0543

ISN 0544

ISN OS45

ISN 0546

ISN 0547

ISN 0548

ISN 0549

ISN 0550

ISN 0551

ISA 0552

ISN 0553

ISN 0554

ISIV US55

ISN 0556

ISN 0557

ISN 3558

ISN 0559

ISN U500

ISN U561

ISA 0562

ISN 0563

ISN 0564

ISN 0565

ISN 0566

ISN 0507

ISN 0568

ISN 0565

ISA $057 \mathrm{~J}$

ISN 0571

$15 N \quad 0572$

ISN 0573

ISA 0574

ISN 0575

ISA 0570

ISN 0577
873 FORMAT(1HO, AEI (FT*2)", IPE12.4,5X, EQUETICN ?1") WRI TE $(6,874) A$ PHI

874 FCRNAT(IHO, 'APHI (FT* Z)', IPE12.4,5X, 'EQUATION 22') WRITE $(6,875)$ A I

875 FORMAT(IHO.'AI (FT*2)'.IPEL2.4.5X.' FQUATICN 23') WRITE 10,876$) \triangle B 2$

E7O FORMAT(IHU,'AB2 (FT*2)', LPE12.4,5X,' EQUATION 24') WRITE $(6,877) \mathrm{APH} 2$

677 FCRMAT(1HO, 'APH2 (FT*\# C)', IPE12.4, DX, 'EQUATION 25') WRITE $(6,87 E) A 2$

878 FORMAT(1HO,'A2 (FT* 2 )', IPE 12.4,5X, 'EQUATICA 26 ') WKITE 0,875$)$ ASUM

E79 FCRMAT(1HU,1ASLM IFT*2)'.1PE12.4.5X.'EGUATION $27 \cdot 1$ WRITE $(6,880)$ AC

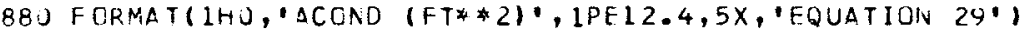
WRITE $(6,881)$ HTA

EE 1 FORMAT(1HO, "HTA (HT* 21$)$, IPE12.4,5X,'EQUATICN 30') WRITE $(6,882) \mathrm{WC}$ W

882 FCRMAT(1HO, 'WCW (LE/HR)', IPE 12.4, 5X, 'EQUATICN 31 ') IVITE $\{6,883) G P M$

883 FGRMAT( 1 HO.'GPM (GAL/MIN) ', IPE $12.4 .5 \mathrm{X}$, 'EQUATIONN 32 ' WRITE $(6,884)$ QCOND

884 FORMA T(1H), OOC IND (BTU/HR)', 1PE 12,4,5X, 'EQUATION 28 ') WRITE $(0.885)$ WFI

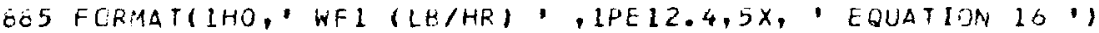
WKITE $(6,886)$ TRGO

886 FORMATI IHO,' TEMP REG CUT ', IPE12.4,5X,' DEg F.' WRITE $(6,887)$ TB5

E\$7 FORMATIIHJ.' TEMP BR OT ' .1PE12.4.5X.' DEG F' ' NPITE $(6,8$ B) UELRG

Q88 FORMAT(IHU,' DEL ENTH RGT ',LPEL2.4,5X,' ETU/LB' I WRITE (6,8 QS) ARG

GE9 FURMAT(1HO,' REG AREA ', LPF12.4,5X,' FT SQ') WRITE (́,890) DLTPPI

69O FORMAT(1HO,. PIN PT 1 , , 1PE12.4,5X,' DEG F ' WRITE $(6,851)$ DLTPPZ

ESI FGRMAT(1HJ.' PIN PT 2 . .IPE12.4.5X.' CEG F', WRITE $(6,8 \$ 2)$ TBIP

992 FCRMATIIHU,'BRINE SUPERHEAT 1 EXIT',1PE12.4,5X, "DEG F') WRITE $(6,853)$ TB3P

E\$3 FORMATILHO, GRINE SUPERHEAT 2 FXIT',1PE12.4,5X, DEG F') WRITE (S,E94) HII

ES4 FERMATILHO,' TUR ONE ENTH IN', IPE12.4,5X, 'BTU/LB') WP!TE (6, E क5) HI2

ES5 FORMAT(IHO,' TLR THC EATH IN', IPEI2.4.5X. 'RTU/LB') WRITE $(6,890) \triangle 5 H 1$

896 FURMAT(1HU,' DREA SUPHEAT ONE', IPEL2.4,5X, 'FTSQ' I WRITE $(0,897)$ ASH2

EC.7 FOKMAT(1HO,' AREA SUPFEAT TWO',IPE12.4,5X, ' FTSQ' 1 WRITE $(6,898)$ QALN

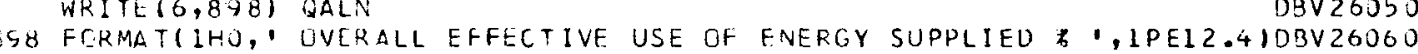
WRITE $(O, 8 S 9) U B P$

ECS FORMATI 1HJ.' BRINE PUMFING POWER , 1PF12.4.5X.' MW 11 HRITE 10,500$)$ WW

CJu fORMAT(IHJ,' CONJEASING WLTER PUMPING POWER, 1 PE 12.4,5X, MW' WRITE $(6,901)$ QFP
DBV 25560

DBV 25570

OBV 25580

DR 25590

DBV 25600

DRV 25610

DBV 25620

DBV 25630

DBV 25640

DBV 25650

DRV 25660

DRV 25680

DBV 25690

OBV2570J

DBV 25710

DRV 25720

DBV 2573 J

DBV 25740

DBV 25750

DAV 25760

OBV 25770

DBV 25780

DRV 25790

กBV 25800

DRV 25810

DAV 25820

DBV 25830

DRV 25840

UBV 25850

DBV 25860

DBV 25870

UBV 25880

DBV 25990

DBV 25900

DBV 25910

DBV 25920

DBV 25930

DBV 25940

DBV 25950

DBV 25960

DBV 25970

DBV 25980

LAV 25990

DSV 26000

1) $8 \vee 26010$

DRY 26020

DBV 26030

DRV 26340

$0 B \vee 26: 70$

DRV 26080 DRV 26000

DBV 26100

DAV 26110
DBV 25670 


\begin{tabular}{|c|c|}
\hline $5 N$ & $\begin{array}{l}0578 \\
0579 \\
0580\end{array}$ \\
\hline & 4301 \\
\hline & 0582 \\
\hline & 0583 \\
\hline & \\
\hline & 358 \\
\hline & \\
\hline & 0587 \\
\hline & 0588 \\
\hline A & 0589 \\
\hline & 590 \\
\hline & 0591 \\
\hline & 0552 \\
\hline & 0553 \\
\hline & 0594 \\
\hline & 0505 \\
\hline & 0556 \\
\hline & C5s7 \\
\hline & 0558 \\
\hline & 0595 \\
\hline & 0600 \\
\hline & 0001 \\
\hline & 0602 \\
\hline & 0603 \\
\hline & 0604 \\
\hline & 0605 \\
\hline & i) \\
\hline & C6 \\
\hline & 06 \\
\hline & 00 \\
\hline & $0 \in 10$ \\
\hline & $0 \in 11$ \\
\hline & $j \in 1$ \\
\hline & \\
\hline & \\
\hline & \\
\hline & \\
\hline & 06 \\
\hline & \\
\hline & \\
\hline & \\
\hline & 24 \\
\hline & 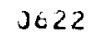 \\
\hline & \\
\hline & $c \in 2$ \\
\hline & $j t$ \\
\hline & $0 t$ \\
\hline
\end{tabular}

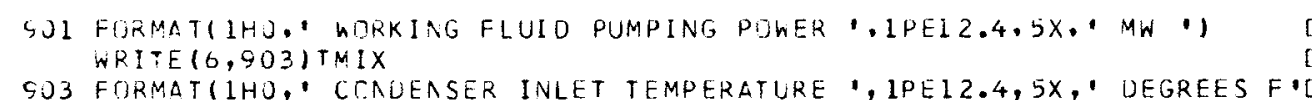
11 INR ITE $(6,902) \mathrm{NCW}$

C.J2 FGRMAT(IHU," NUMBER OF BRINE SUPPLY WELLS " IS) NRITE (6,904) NUBWP.

SO4 FORMLT(1HO," NUMBER CF BRINE REINJECTION WELLS", I5) WKITE $(6.905)$ NDCW

905 FORMATIIHO," NUMBER CF CDOLING WATER SUPPLY WELLS', I5) WRITE $(6,906)$ NECWR

500 FORMAT(1HO,' NUMBER CF COOLING WATER REIAJECTION WELLS', 15) NFITË $(6,507)$ PF 1

SUT FORMAT(IHO,'PRESSURE HP BOILER', IPE12.4,5X, 'PROP DECK') NRITE $(6,908)$ PF2

SO8 FORMAT(1HO,'PRESSURE LP BOILER', 1PE12.4,5X, 'PROP DECK') WFITE $(6,905)$ PCCND

SUS FURMATIIHJ, 'CLNOENSING PRESSIJRE', IPEI2.4,5X,'PRCP DECK'I WRITE $(6,910) \mathrm{AP}$

\$1 J FORMAT( 1 HO, 'PUNP EFFICIENCY',1PE12.4,5X, 'INPUT') WRITE $(6,911) \mathrm{NEL}$

$\$ 11$ FURMAT(1HO, 'ELECTRICAL EFFICIENCY', IPE12.4,5X, 'INPUT') WRITE (Ó,912)NGTI

S12 FIJRMAT( IHO, 'HP TURBINE EFFICIEN!CY',1PEI2.4,5X, 'INPUT') WRITE $(6.913)$ NGT2

$\$ 13$ FORMAT(1HO, 'LP TURBINE EFFICIENCY',1PEI2.4,5X,'INPUT'I WRITE $(6,914) \mathrm{WBW}$

\$I 4 FCRMAT(IHJ,'MAX FLChRATE / RRINE SUPPLY WELL', 1PE12.4,5X,'LBN/HR') WRITE 6,915 ICONDH

S15 FORMATI1HU, SAT VAFCR ENTHALPY AT CONL TFMP', IPE12.4.5X, IPROP DECK I') WRITE $(6,958)$

DBV 20240

S48 FORMATLEX, TEMP BR INLET TP BR SPH 1 OT TP BR PRH I OT 1 TP BR SPH 2 OT TP BR OUTLET BRINE FLOW RATE 'I WRITE $(6,1000) T B 1, T B 1 P, T B 3, T B 3 P, T B 5, W B$

1000 FCRMAT $(1 \times .6(2 \times \cdot E 15.4 .3 \times))$ WRITE 16,1200$)$

1200 FOKMAT16X, TP TURI INLET EN TURI INLET TP BOILER DNE 1 FLOWRT TR ONE EN EST BOIL 1 EN ACT EX TURI $)$ WRITE $(6,1000)$ TI1, HIL, TF1, WFI, HG I, HGITO WRITE(6,1300)

IEOO FORMATLGX, TP TUR2 INLET EN TUR2 INLET TP BOILER TWD

1 FLOW RT TR TWO EN EXT BCIL 2 EN ACT EX TUK2 1 WRITE $(5,1000)$ T12.HI2,TF2,WF2.HG2,HG2TO NRITE (6,1400)

1400 FURMAT(OX, LREA ECILER 1 AREA BOILER 2 AREA PREHET 1 1 AREA PREHET 2 AREA CONDENSER AREA REGENATOR 'I WRITE $(5,1000)$ AB1, AB2, APH1, APH2, AC, ARG WRITE $(6,1500)$

1500 FORMAT( $6 X$, " AREA SLPHET 1 AREA SUPHET 2 DELT PIN PT 1 1 DELT PIN PT 2 TEMP REG OUT FLOW RATE COOL ') WRI TE $(6,1,000)$ ASH1, ASH2, DLTPP1, DLTPP2, TRGO, WCW NPCINT $=$ NPOINT +1 IFINSUBSC EQ. O) GO TC 104 TEMPL T(NPOINT) $=A(N S U B S C)$ RATPLT(NPOINT) =RAIIC 3
DBV 26120 OBV 26130 DRV 26150 DBV?6100 DBV 26170 DRV 26180 DBV 26190 DBV 26200 OBV 26210 DBV 26220 DPV 26230

J $A \vee 26250$

Dค 26260

DBV 26270

DPV 26280

กBV26290

DPV 20300

DAV 26310

D 826320

DRV 26330

DBV 26340

D8V26350

DBV 26360

DBV 26370

DBV 26380

DBV 26390

DBV264J

D果 26410

DBV 26420

DBV 26430

DBV 26440

DEV 26450

OBV 26460

DAV 26470

DBV 26480 
ISN 0620

ISN DE 29

ISN 0631

ISN OE 32

ISN $0 \in 33$

ISA OE 34

ISN $0 \in 35$

ISN $U E 36$

ISN OE 37

ISN 0638

ISN $0 E 39$

ISN OE40

ISN DE 41

ISN DE 42

ISN $0 \in 43$

ISN OE 44

ISN 0645

ISN 0646

ISN 0647

ISN 0048

ISN 0649

ISN 0650

ISN DEEI

ISN $D E 52$

ISN OU 54

ISN JE55

ISN 0656

ISN 0657

ISN 0658

ISN 0659

ISN 0660

ISN 066 I

ISN $06 t 2$

ISN 0663

ISN 0664

ISN 0665

ISN 0660

ISN $0660^{\circ}$

ISA 0665

ISN 0670

ISN 0671

ISiN 0672

ISN 0673

ISiN 2674

IS N 3675
104 CONTINUE

IF (RRAT .EQ. 100.) GC TO 106

DO $105 \quad I=1,50$

$A(I)=C(I)$

105 CONTINUE

100 CONTINUE

c

$H E X C O S=10.56 *(A E 2+A E 1)+6.91 *(A P H 1+A P H 2+A R G+A S H)$

TRBCDS $=8268.5 *(P C, R C S S * 1000.1 * .53$

SECCOS $=2032.0 *(P G R C S S * 1000) * *$.

ELCCOS $=18835$. $($ PGRCSS*1000. $) * .42$

$C D C D S=7.600 * A C+24.25 * W C W * .65$

EQCOS = HEXCOS+COCOS +ELCCOS + SECCOS+TRBCCS

$S P L Y C D=.0383 * W B * P N E T$

TOTSYS $=1.68 * E Q C C S+S P L Y C O$

COSPGW $=1.03436272 *$ EQCOS $+.022966 *$ SPLYCO) /(PNET*1000.1

$3 P L Y C I=N U W * 284 \mathrm{JJ}$. \#PNET

TOTSYI $=1.68 *$ EQCCS+SFLYCI

$\operatorname{CCSPOI}=1.03436272$ EQLCD $+.022968 * S P L Y C I) /(P N E T * 1000$. WRITE $(0.1235)$

1235 FORMATI

1 COCTCSI SPLYCST TOTSCST CSTPOW MILS PR KWHR '

2)

WRITE $(6,1234) \mathrm{HEXCOS,} \mathrm{TRBCOS,} \mathrm{SECCOS,} \mathrm{ELCCOS,} \mathrm{CDCOS,} \mathrm{SPLYCO,} \mathrm{TOTSYS,} \mathrm{COSPD}$ IW

WRITE $(0,1234)$ HEXC CS. TRBCOS, SECCOS, ELCCOS, CDCOS, SPLYCI, TOTSYI, COSPO 11

1234 FORMAT( BD15.4)

C TEST PLOT SELECT SWITCH AND SUBSCR VALUES

IF (NSUBSC, .EQ. O .OR. NPLTSW .EQ. O) GC TC 4238

$M I N T=1 \cdot E+C$

MAXT $=0.0$

$M I N 1=0$.

$\operatorname{MAX} 1=-100$.

DO $2 J 1 N=1, N P C I N T$

MINT = AMINI (TENPLT (N), MINT)

$M A X I=\angle M \triangle X 1(R A T P L T(A), M A X I)$

MAXT = AMAXI(TEMPLT(N), MAXT)

201 CONTINUE

$M I N T=D E \angle T * \triangle I N T(N I N T / O E \angle T)-O E L T$

MAXT $=$ DE $Z T * A I N T(M A X T / D E Z T)+D E Z T$

MAXI $=1 U . * A I N T(M \Delta X 1 / 1 J .1+1 J$.

IF I IPLT.EO. O) CALL MDOESG(Z.O)

$I P L T=1$

CALL SUBJEGIL, MINT, NINI, MAXT,MAXI)

CALL CBJCTC $(\angle \ldots 1, .1 \ldots 8, .97)$

OEZU $=D E Z T / 2$

CALL GRIUG $12, U E Z U, 2,2,2,51$

CALL LDEELG(L,O,DEZT, 0,7.2)

CALL LABELC $(2,1,5.0,3,5.2)$

DBV 26490

DBV 26500

DBV 26510

DBV 26520

DBV 26530

DBV 26540

DBV 26550

DBV26560

DBV26570

DBV 26580

DB 26590

DBV 26600

DBV26610

DBV26620

DRV 26630

DBV 26640

DBV 26650

DRV 26660

D 8 V26670

D 8 V 26680

USV26690

DB V 26700

DBV 26710

DBV 26720

UAV 26730

DRV 26740

DBV $2675 \mathrm{~J}$

DBV 26760

DAV 26770

DBV26785

CALL TITLEG $2,40$. GRTITL.33.

*, 33HSENSITIVITY STLOY FOR $50 \mathrm{MW}$ PLANTI

กBV 26750

DBV 26800

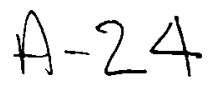


ISA 0070

ISN 0677

ISN 0678

ISN 0675

ISN 2680

ISN 0681

ISA OOEE

ISN 0683

ISN 0685

ISN 0687

ISN JOEE

ISA 0650

ISA $26 S 1$
CALL SETSMG $(2,52,1.5)$

CALL SETSMG $(2,55,2.0)$

CALL SETSMGIL,84.1 HK)

CALL POINTGIZ, NPCINT, TEMPLT, RATPLTI

CALL PAGEG $(2,0,0,1)$

4238 CONTINUE

4239 CONTINUE

IF (NSTOP . LT. 2 .CR. NSTOP .EQ. 6) GJ TO 4250

IF (NCASE. NE. O) GE IC 4

GC IO $b$

4250 IF IIPLT.NE. O) CALLEXITGIL) STOP

END
DBV26810

DBV 26820

DBV 26830

DEY 26640

DBV 23850

DRV 26860

DRV 26870

DBV 26880

DBV26890

DBV 26900

DRV 26510

UBV 26920

DBV 26930

$$
\text { A-25 }
$$




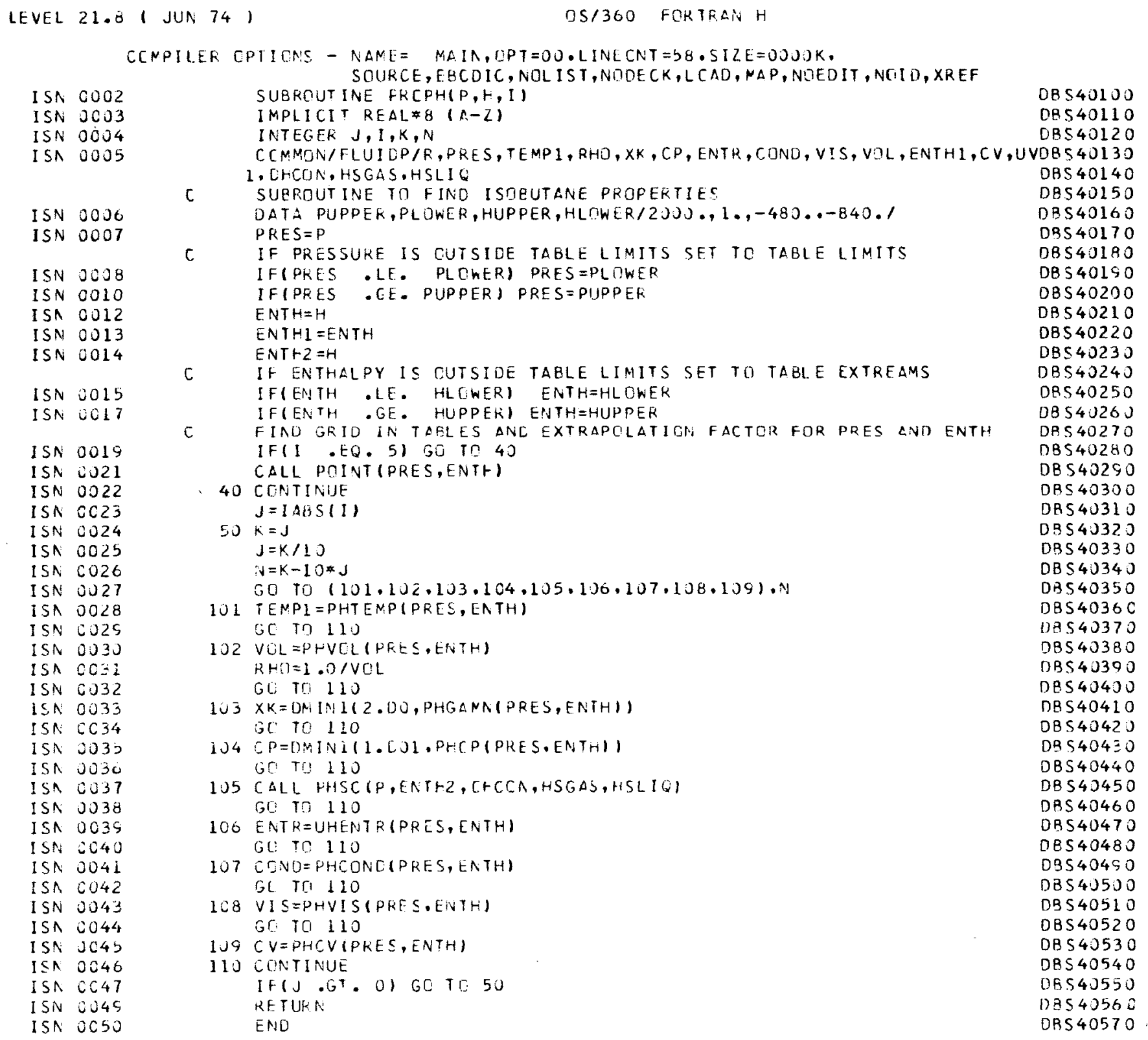

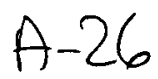


CCNPILER CPTIONS - NAME = MAIN, CPT=0J, LINECNT=59, SIZE=0OOJK,

ISN 0002

ISN CUUB

I $S N \quad 0004$

ISN 0005

ISN 0OOÓ

ISN 0007

ISA $\operatorname{CCOS}$

I SiN OOUS

ISA COIO

ISN 0011

ISN 0012

ISN DOI3

ISA 0014

ISN 0015

ISN 0016

ISN 0017

ISA 0018

ISN OUIS

ISA CO2O

ISN 0021

ISN 0022

ISN 0023

ISN 0024

ISN 0025

ISN CC26

ISN 0027

ISN $00 \angle 9$

ISN 0030

ISN 0032

ISN 0033

ISN 0034 SUBROUT INE PLINT $(P, H)$

I MPLTCIT REAL $8(A-Z)$

C SURFOUTINE TO OETAIN DESIRED GRID SQUARE FIIR PRIPERTIES CALCULATIODBS4O6OO INTEGER I, J, IC, JO, NPR, $A$ EN

CEMNON/POIN/NPR,NEA.FI,FJ.IC,JG.I.J

DIMENSICN PR (25), EN(25) $\quad$ DBS40630

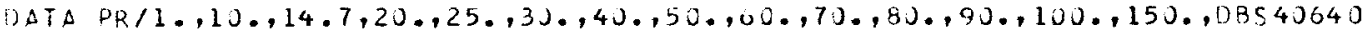

$1200 . .250 . .300 . .400 ., 500.0600 .800, .1000 ., 2000.1 \quad$ DRS40650

$\begin{array}{lll}\mathrm{JPK}=23 & \text { DBS4J660 }\end{array}$

DATA $[1 /-840,,-820,9-800,9-780,,-760,,-740,,-720, \ldots-700,,-680, \ldots-66008540670$

$1 .,-640 .,-620 .,-600 .,-580 .,-560 .,-540 .,-520,-500 .,-480.1 \quad$ DBS40680

$N E N=19$

$\mathrm{F} I=1$

$F J=1$

C FIND SUESCRIPT POIAT THAT BDUNOS PRESSURE

DU 3. $I=1, N P R$

$! n=1$

If $(P F(1)-P) 30,40,50$

30 CONTINUE

C IF PRESSURE IS EXECTLY CN GRIO SET ENTHALPY FACTOR TO LERO

DB 540690

DB 540700

DBS40710

DES4J72D

DAS40730

08540740

DBS40750

DRS 40760

DB 540770

08540780

DBS40790

DSS40800

DBS40810

DBS40820

DBS 540830

DR540840

DBS4 4850

DB 540860

DBS40870

DR $\$ 40880$

DBS 40850

DBS40900

DBS4091D

DP. 540920

DBS $\$ 40930$

DR $\$ 40940$

DBS40950

140 KETURN

END

DR 540960 
LEVEL 21.8 ( JUN 7K)

OS/360 FIRTRAN H

CCNPILEK DPTIONS - NAME = MAIN, OPT =00, LINECNT $=58$, SIZE=0000K, SRURCE, EBCDIC, NOL IST, NOUECK, LCAD, MAP, NDEDIT, NOID, XKEF

ISN 3002

ISA 0003

ISN 0004

ISN 0005

ISN COOS

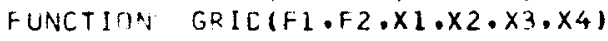

DB 540570 IMPLICIT: REAL $\$ 8(A-Z)$

C CCMPUTATIIN IF LINEAR INTERFOLATION FGR TWO DIMENSION TABLES $G R 1 D=X 1 *(1-F 1-F 2+F 1 * F 2)+X 2 * F 1 *(1.0-F 2)+X 3 * F 2 *(1.0-F 1)+X 4 * F 1 * F 2$ RETURA

END

DBS40980

DB $\$ 40990$

03541000

DBS41010

DBS $41 J 20$ 
CCMPILER CPTIONS - NAME $=$ MAIN, OPT $=00$, LINECNT $=58, S I Z E=0000 \mathrm{~K}$, SCURCE, EBCDIC, NOL IST, NODECK, LOAD, MAP, NOEDIT, NOID, XRFF

ISN 0002

ISN 0003

ISN COC4

ISN 0005

ISN 0000

ISN 0007

ISN 0008

ISN COOS

ISN 0010

ISA COLI

ISN 0013

ISN 0015

ISN 0016

ISN CU17

ISN OOIB

ISN COIS

I ISN 0020

ISN 0021
FUNCTION PHTEMP(PRES, ENTHI

IMPLICIT REAL $\$ 8$ (A-L)

INTEGER IC, JO, I, J, NPR, NEN

C CMNCN/POIN/NPR, NEN, FI, FJ,IO.JO,I J

DIMENSIGN TEMP $(23,1$ S), TEMI(161), TEMZ(161), TEM3(115)

DB $\$ 41030$

DBS 41040

DBS41050

DBS41360

DB $\$ 41070$

EQUIV $\triangle L E N C E$ (TEMP(1,1), TEMI (1)), (TFMP $(1,8), \operatorname{TEM} 2(1)),(T E M P(1,15), T E D B 541080$

$143(1))$

FUNCT ION TO FIND TEMPERATURE FROM PRESSURE AND ENTHALPY

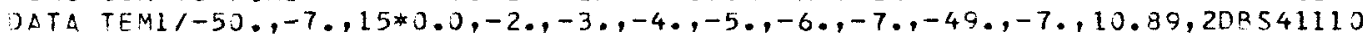
$16 \cdot 5 \cdot 37.5 \cdot 37.5,38 \cdot .36 \cdot 3,2 * 36.3,35.5,9 * 35 ., 30,20,10,,-48,,-7,10$ DBS 41120 $2.89,26.5,37.5,47.5,63 ., 71 \cdot 3,71.4,71.5,6 * 71 \ldots, 70.5,6 * 70 .,-47,,-7 ., 1008541130$ $3.89,26.5,37.5,47.5,63 ., 77 ., 89 ., 98 \cdot 5,7 * 135 \ldots 3 * 104 \ldots 3 * 103 \ldots-46 \ldots-7.08541140$ $410.89,26,5,37,5,47,5,63 ., 77 ., 89 ., 58.5,107 ., 115.5,123 \ldots 137 \ldots, 3 * 136.503541150$ $5,136 ., 135 ., 135 ., 3 * 136 \ldots,-45 \ldots,-7 ., 10.89,26.5,37.5,47.5,63 ., 77 \ldots, 89 .$, DBS41160 $658.5,107 \ldots 115.5,123 \ldots 154 \ldots 3 \times 166 \ldots 4167 \ldots 166.5 .166 \ldots-44, .-7 \ldots 10.89,08541170$ $726.5,37.5,47,5,63 \ldots, 77 \ldots, 89,98.5,107,115.5,123,155,178.5,193 \ldots, 10 B 541180$ $893.5,194 ., 155,195.5,196 ., 197 ., 199.1$ DRS41190

DATA TEM21-42.2.-6.67,10.89,25.6,36.93,46.67,62.88,76.35,87.89,98. DBS41200 $103,107.2,115.5,123.1,154.4,178.5,198.7,216.1,220.0,221.0,222 ., 224.0 B 541210$ $2,224 \cdot 5,226 \cdot, 2 \cdot 5,5.0,10.89,25.6,36.53,46.67,62.88,76 \cdot 35 \cdot 87.89 .98 .0308541220$ $3,107.2,115.5,123.1,154.4,178.5,198.7,216.1,243,, 246,, 249,1252,, 2550 R 541230$ $4 ., 259.9,55 \cdot 3,60 ., 58.23,59.9,60 ., 63 ., 68 ., 76.35,87.89,98.03,107.2,110 B 541240$ $55.5 .123 .1 .124 \cdot 4.178 \cdot 5 \cdot 198.7 \cdot 216.1,244.9 \cdot 265.0272 .1280 ., 283 \cdot 7,291.10 B 541250$ $6,104.4,106 \ldots 107.1,108 ., 110, .111 \ldots, 114 \ldots, 115.9,118 ., 120.5,124 \ldots, 126 .$, DBS41260 $7128.5,154.4,178.5,198.7,216.1,244.9,268.7,283.3,298.3,305.6,318 \cdot 1$, DBS41270 $8150.3,152 \ldots 152.8,154 \ldots 155, \ldots 156,, 158 \ldots 159.8,162,164,, 166,168 \ldots, 1690 B 541280$ $9.5,174.6,150 ., 201 ., 216.1,244.9,268 \cdot 7,290.7,313.7,325.3,344.7,194 .$, DBS41290 $1155.9,156.2,157,, 197.5,198,200 \ldots 201.6,204 \ldots 205 \ldots 207 \ldots 208 \ldots 209.5,208541300$ $217.5,225.8,234.3,243.3,262.3,232.7,301.2,328.9,344 ., 370.6,235.6,2304541310$ $37.3,237.3,237 \cdot 1,237.4,238,240,241.8,243.5,244 \cdot 1,246 ., 247.5,248.508541320$ $4.255 .2 .262 .269 .276 .1 \cdot 290.7 \cdot 304.8 \cdot 319.2 \cdot 345.6 \cdot 362.9 .395 .91$ DB 541330 ULTA TEM3/274.5,275.8,276.3,276.9,277.4,278.0,279.1,280.2,281.3,280BS41340 $12.4,283.6,284.7,285.8,291.4,296.8,302.3,307.8,319.1,330.9,342.5,3608 \$ 41350$ $25.3 .383 .2 \cdot 42 \mathrm{i} \cdot 0.312 \cdot 5 \cdot 313.2 \cdot 313.6 \cdot 314.1 \cdot 314.6,315.0,316.0,316.9,3108541360$ $37.0,318.7,315.6,320 \cdot 5,321.4,326 \cdot 0,330 \cdot 6,335.2,339 \cdot 6,34 \% \cdot 3,359 \cdot 0,3608541370$ $48.7,388.4,405.3,445.0,348 \cdot 5,349.2,349 \cdot 6,350.0 \cdot 350.4,350.8,351.5,3508541380$ $52.3,353.1,359.8,354.6,355.3,356.1,360.0,364.0,367.9,371.8,380.2,3808541390$ $68.5,397.0,414.2,423.3,468.9,383.5,384 \cdot 0,384.2,394.6,384.9,385.2,3808 \$ 41400$ $75.5 .386 \cdot 6 \cdot 387.3 .387 .9 \cdot 388 \cdot 6 \cdot 389 \cdot 2 \cdot 389 \cdot 9 \cdot 393 \cdot 4 \cdot 394 \cdot 4 \cdot 400 \cdot 3 \cdot 403 \cdot 9 \cdot 4108541410$ $81.4,418 \cdot 5,423 \cdot 5,433.5,443 \cdot 9,493 \cdot 8,417 \cdot 0,417 \cdot 5,417.8,418 \cdot 1,418 \cdot 4,41$ DBS 41420 $53.8,419 \cdot 4,420 \cdot 0,420 \cdot 7,421 \cdot 4,422 \cdot 1,422 \cdot 8,423 \cdot 5,426 \cdot 5,429 \cdot 5,432 \cdot 5,430 B 541430$ $15.8,442 \cdot 5 \cdot 449 \cdot 1,453 \cdot 3,461 \cdot 8 \cdot 470 \cdot 3 \cdot 512 \cdot 8 /$

$[F(10 . E Q .(N P R+1)) \quad I C=N P R$

IF (JO.EQ. (NEN+L)) JD=NEN

$X 1=\operatorname{TEMP}(1, J)$

$\times 2=\operatorname{TEMP}(1[, J)$

$X 3=T E M P(I, J C)$

$x 4=\operatorname{TEMP}(10, J[)$

PHTEMP $=$ CRICI $(F I, F J, \times 1, \times 2, \times 3, \times 4)$

RETLRN

END
DS541440

DBS41450 DBS41460 DB $\$ 41470$ $D B \subseteq 41480$ DBS 41490 DBS 41500 DBS $\$ 1510$ DRS41520 DBS41530 
CCNPILEF CPTIONS - NAME = MAIN,OPT = JU, LINECNT $=53$. SILE=0OJOK. SOURCE, EBCDIC, NOL IST, NUDECK,LCAD, MAP, NDEDIT, NOID, XREF

ISN 6002 ISN 0003 ISN 0004 ISN 0005 ISN 3006 ISN 0007 ISN 0008 ISN 0005

ISN 0010

ISN UUII

ISN 0012 FUNCTION PHVCL, (PRES, ENTH) IMPLICIT REAL $\$ Q(A-Z)$

INTEGER IO, JO, I, J, NPR, AEN

COMMON/PGIIN/NPR, NEA, FI,FJ, IO, JO,I,J

UIMENSION VOL $(23,15), V C 1(161), V O 2(161)$

JIMENSICN VC3 (115)

EQUIVALENCE (VDL(1.1).VOI(1)).(VCL(1.8).VQ2(1))

EQUIVALENCE (VCL $(1,15)$, VO3(1))

C FUNCTION TO FINC SPECIFIC VOLUNE FROM PRESSURE AND ENTHALPY DATA VDL1/

\& $34.00, .2000, .0267, .0267, .0267, .0267, .0267, .0267, .0267, .0266$,

$\varepsilon .0265, .0266, .0265, .0265, .0264, .0263, .0261, .0257, .0251, .0242$.

\&. $.0232, .0220, .0208,42.0,1.200, .6000, .2300, .0278, .0278, .0278$,

\& .0278,.0278,.0278,.0278,.0278,.00277,.00277,.00276,.0276,.0275,

$\varepsilon .0274 \ldots .0273 \ldots .0272 \ldots 0270 \ldots 0266 \ldots .0260,46.00 .2 .230 .1 .360, .8000$.

$\varepsilon .5050, .3150, .1000, .0289, .0289, .0289, .0289, .0289, .0289, .0288$,

$\varepsilon .0288, .0287, .0287, .0286, .0285, .0284, .0283, .0281, .0277,50.00$,

$\varepsilon \quad 3.350 .2 .000 .1 .330, .9500, .7110, .3820, .2380, .1330, .0660, .0302$,

\&. .0302,.0302,.0302,.0301,.0300,.0299,.0258,.0277,.00296,

$\varepsilon .0295, .0253, .0284,55.00,4.473,2.710,1.870,1.380 .1 .080, .7080$.

$\varepsilon_{2} .4900, .3400, .2580, .1870, .1310, .0559, .0316, .0316, .0315, .0314$,

$\varepsilon .0312, .0211, .0305, .0367, .0305, .0255,62.30,5.413,3.470$,

c $2.380 .1 .840 .1 .470 .1 .000 .07490 . .5610 . .4390 . .3550 . .2500, .2320$,

c. $.0715, .0335, .0334, .0332, .0330, .0327, .0325, .0321, .0318, .0306$,

\& 7J.0u,6.330,4.130,2.930,2.280,1.850,1.31 J,1.0J J,.7850,.6320,

E. .5120..4310,.3690,.1700..0770..0358..0355..0350,.0347,.0344, \&. $.0339, .0335, .03121$ DATA VחLI

$\varepsilon 77.40 .7 .200,4.880 \cdot 3.450,2.740,2.240,1.610,1.250,1.000, .8310$, $\varepsilon .6690, .5920, .5000, .2710, .1550, .8500, .0448, .0390, .0375, .0371$, $\varepsilon .0362, .0354, .0335,84.60 .8 .060 .5 .640 .4 .340 .3 .210,2.650 .1 .930$. $\varepsilon \quad 1.500,1.220,1.020, .8600, .7420, .6510, .3780, .2350, .1540, .1000$, E. .0425,.0417,.0404,.0391,.0 J382,.0350,55.50,8.790,6.310,4.490, ₹ $3.650 .3 .060 .2 .220,1.720 .1 .430 .1 .180 .1 .320 .08910 . .7840 .04680$. E .3090,.2190,.155c,.0810,.0400,.0474,.0440,.0417,.0369,103.0, \& $9.500,0.930,4.940,4.060,3.350,2.500,1.950,1.6) 0,1.340,1.150$,

\& $1.030 .5070, .5640, .3850, .2810, .2110, .1400, .0770, .0541, .0497$. $\varepsilon .0465, .0380,112.0,10.20,7.540,5.400,4.370,3.640,2.7 J 0,2.140$,

$\varepsilon \quad 1.770,1.510,1.280,1.140 .1 .020, .6450, .4560, .3350, .2640, .1680$,

s. .1120,.0864,.0555,.0521,.0411,120.0,10.90,8.120,5.870,4.680,

\& $3.930,2.900,2.320,1.530,1.630,1.410,1.200,1.120, .7110, .5120$,

$\varepsilon .3890 . .3110 . .2070 .1480, .1089,00735 \ldots 0600 \ldots 0439.128 .0 .11 .50$.

\& $8.620,6.260,4.590,4.170,3.100,2.500,2.130,1.760,1.530,1.350$,

E. $1.210, .7740, .5600, .4300, .3460, .2360, .1760, .1348, .0891, .0710$,

$\gamma_{2} .04671$

DATA VISI

\& $136.0,12.00,9.130,6.630,3.3 .00,4.390,3.300,2.650,2.210 .1 .880$. $\varepsilon \quad 1.630,1.460,1.300, .8310, .6070, .4670, .3810, .2650, .2020, .1575$,

$\varepsilon .1058, .0815, .0496,142.0,12.40,9.640,7.030,5.610,4.620,3.500$,

$\varepsilon \quad 2.800 .2 .350 .2 .000,1.730,1.550,1.390 . .88400 .0480, .5040 . .4130$.

\&.2940,.2210,.1768,.1206,.0841,.0531,149.0,12.80,10.00,7.420,

$\varepsilon 5.920,4.840,3.090,2.940,2.490,2.10 J, 1.830,1.630,1.480, .9360$,

\&.5880,.5380,.4390,.3160,.2410,.1558,.1354,.1043,.0508,154.0.

\& $13.20,10.50,7.830,6.150 .5 .070,3.890,3.050 .2 .590 .2 .220,1.930$,
DBS 41540 DBS 41550 DBS41560 DBS41570 DRS 41580 DB 541590 DBS 541600 DSS41610 DBS4162J DBS 41630 UBS 41640 DBS41650 DBS41660 DBS 41670 DBS41680 DR $\$ 41690$ DRS41700 DBS41710 DBS41720 DBS 41730 DBS 41740 DB\$41750 DBS41760 DRS41770 DBS 41780 DRS 41790 OBS41800 DBS41810

DBS41820

DBS41830

OBS 41840 DBS41850

DR $\$ 41860$

DB $\$ 41870$

UBS41380

DBS 41890

DBS 41900

DBS41910

$D B \subseteq 41920$

DBS41930

DBS4194J

DS\$ 41950

DBS41960

$D B \subseteq 41970$

DBS41980

DBS41990

DBS 42010

DRS 42010

DBS 42320

DB 542030

DeS 542040

DS542J5J

DBS42060

DBS42.070

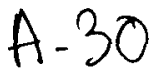


ISiv 0013

ISA OOLS

ISA 0017

ISN 0018

ISN OOLS

ISN CO20

ISN 0021

ISA $0=22$

ISA 0023
E $1.710,1.500, .9860, .7300, .5720, .4070, .3380, .2610, .2100, .1500$,

$\varepsilon .1150,00610,102.0,13.60,11.00,8.150,6.430,5.300,4.060,3.190$.

\& $\angle .200,2.320,2.020,1.750,1.590,1.030, .7710 .6000$.

\&.4940,.5560,.2800,.2230,.1610,.1290,.00501

IF(ID. EQ. (NFP+1)) IC $=N P R$

IF(JO EQ. (NEN+1)) JO=NEN

$X 1=\operatorname{VOL}(I, J)$

$\times 2=V O L(I 0, J)$

$x 3=V D(I, J C)$

$X 4=V O L(I O, J C)$

$P H V O L=G R I D(F I, F J, \times I, \times 2, \times 3, \times 4)$

KETURA:

END
DBS $\$ 2090$

DBS42090

DBS 421$) 0$

DBS42110

DBS42120

DBS42130

DRS42140

DBS 42150

DBS $\$ 2160$

UB $\$ 42170$

DBS42180

DBS42190

DBS4?200 
EEVEL $21.8(J L N 74)$

DS/30O FORTRAN H

CCNPILER CPTIONS - NAME $=$ MAIN,OPT=0O, LINECNT $=58$, SIZE $=0000 \mathrm{~K}$, SOURCE, ERCDIC, NOLIST, NOOECK, L CAD, MAP, NOEDIT, NOIO, XREF

ISN 0002

ISN 0003

ISN 0004

ISN 0005

I SN 0006

ISN 2007

ISN 0008

ISN 0010

ISN 0012

ISA 0013

ISN 0014

I SN 0015

I SN 0016

ISN 0017

I SN COLO
FUNCTION PHGAMN (PRES, EATH)

IMPLICIT REAL $\$(A-Z)$

INTEGER IO, JO, I, J, NFR, NEN

COMMON/POIN/NPR, NEN,FI, FJ, IO,JO,I, J

DIMENSION GAMN $(25,25)$

C FUNCTION TC FIND RATIO OF SPECIFIC HEATS

DATA GAMN/E25*10.1

IF(ID.EQ. (NFR+1)) IC=NPR

IF(JO) EEQ. (NEN+1)) JO=NEN

$X I=\operatorname{GAMN}(I, J)$

$X 2=G A M N(I C, J)$

$X 3=G I M N(1, J E)$

$X x_{4}=G \Delta M N(I C, J U)$

$P H G A M N=G R I L(F I, F J, X 1, \times 2, \times 3, X 4)$

RETUR N

END
DBS 42210

DBS42.220

DBS 42230

ORS 42240

DBS 42250

DB. 542260

DBS 42270

DB $\$ 42280$

UB $\$ 42290$

$D B \$ 42300$

UBS 42310

$D B \subseteq 42320$

DBS42330

DBS42340

DBS 42350

DBS42360 
CCNPILER OPTICNS - NAME = MAIN,CPT=00, LINECNT $=58$, SIZE=0000K, SOURCE, EBCDIC, AOLIST, NDDECK.LCAD, MAP, NDEDIT , NOID, XREF

ISN 0002

ISN 0003

ISA 0004

ISN 0005

ISA 0006

ISN 0007

$15 \wedge \quad 0000$

ISN 0005

ISN COIJ

ISN 0011

ISN 0012

ISN: 0014

ISN 0016

ISA 0017

ISN 0018

ISN OOIG

ISN 0020

ISN 0021

ISN 0022
FUNCT ION PHCP (PKES,ENTH)

INPLICIT REAL*B $(A-Z)$

INTEGER IO,JO,I,J,NFR, AEN

CCMMON/POIN/NPR,NEN,FI,FJ,IC,JO,I, J

OIMENSION CPFN $(23,19), C P F 1(161), C P F 2(161), C P F 3(115)$

EQUIVALENCE (CPFN(1,1),CPF1(1)), (CPFN(1,8),CPF2(1))

EQUIVALENCF (CPFNI1, 15), CPF $3(1)$

C FUNCTION TO FIND CP FROM PRESSURE $\triangle N D$ ENTHALPY

DATE CPFI/3.,.56, 4*.555,8*.55,2*.545,.54,.535,.53,.525,.52,.51,

D8 542370

DB 542380

DBS 42390

DBS42400

DBS 42410

DB 542420

DBS 42430

DB $\$ 42440$

$\varepsilon .505,3 ., .57, .56,2 * .57, .565,11 * .56, .555, .55, .54, .53, .525, .52,4 * 3 ., 0 B 542460$

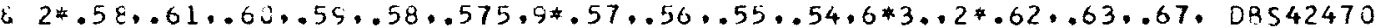

$\varepsilon .66, .65, .64,5 * .61, .55,10 * 3 ., .68, .64, .71, .67,3 * .66,3 * .635,2 * .625$, DBS S42480

$\varepsilon .61,12 * 3 ., .76, .75, .72, .71, .7, .69, .67, .665, .66, .655, .635,13 * 3 ., .790 B S 42490$

$\varepsilon, .78, .77, .76, .755, .74, .73, .70, .69, .6651$ DBS 42500

i) ATA CPF2/.385, .45,.46,11*3.1.8,..79,.83,.815,.785,.75,.715,.69,..66D8S42510

$\varepsilon, .365, .395, .42, .43, .44, .485, .55, .5,8 * 3, . .94, .92, .91, .80, .715, .69$, DBS42520

E. .655,..35,.355,.40, .41,.42,.425,..45,.465,.485,.5, .505,.59, .56,.59, DBS42530

$\varepsilon \quad 3 * 3 ., .96,1 \ldots 1.165, .865, .79, .685,2 * .42,3 * .425, .43, .445, .45, .46, \quad$ DBS42540

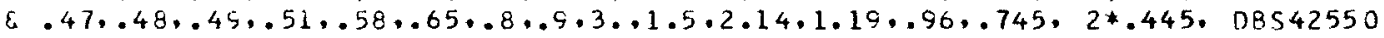

$\varepsilon 2^{4} .45, .455, .46,2+.465, .47, .475, .40, .485, .49, .53, .56, .6, .0675, .92$, DBS42560

\& $1.18,2.24,1.31,1.04, .76,2 * .47, .475, .48, .485,4 * .49,3 * .50, .505, \quad$ DBS 42570

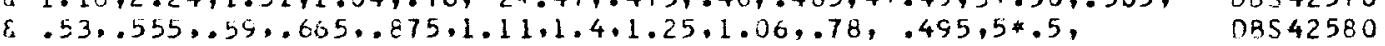

$.505, .51,2 * .515,2 * .52, .525, .54, .565, .59, .64, .705, .83, .97,1.1, \quad$ DBS42590

$\varepsilon 1.02, .821$

DATA CPF3/6*.52,.525,.53,2*.535,.045,2*.55,.565,.585,.66,.63,.68,

$\varepsilon .74, .81, .935, .945, .84,4 * .545,3 * .55,2 * .555, .56,2 * .565, .57, .585$,

$\varepsilon .595, .61, .625 . .655, .655, .735, .82, .85, .86,4 * .565,3 * .57,2 * .575$.

$\varepsilon \quad 3 * .58, .585, .555, .005, .615, .625, .645, .67, .725, .795, .84, .87$

\& $4 * .585,6 * .59,3 * .595, .6, .61, .62, .63, .64, .66, .71, .78, .84, .87$,

E. $5 * .595 .4 * .6 .4 * .605, .615 \ldots 625, .635, .65, .66, .7, .77, .83, .86, .671$

$I F(I O \cdot E Q \cdot(N P R+1)) \quad I C=N P R$

I $F(J] \cdot[Q .(N E N+1)) \quad J C=N E N$

$X 1=C P F N(I, J)$

$x_{2}=C P F N(I C, J)$

$X 3=C P F N(I, J C)$

$X 4=C P F N(I C, J O)$

$P H C P=G R I \cup(F I, F J, \times 1, \times 2, \times 3, \times 4)$

RETURN

END
DBS42600

DBS42610

DBS 52620

DBS42630

DBS42640

$D B \subseteq 42650$

DBS 42660

DBS42670

DBS42680

DBS42690

DB $\$ 42700$

OB $\$ 42710$

DBS 42720

DB $\$ 42730$

DBS42740

DBS42750 
CCNPILEF CPTICNS - NAME = MLIN, OPT $=00$, LINECNT $=58$, SIZE $=0000 \mathrm{~K}$, SOURCE, EECDIC, NOL IS T, NODECK, LCAD, MAP, NOEDIT, NOIO, XREF

ISN 0002

ISN 0003

ISN OCO4

ISN 3005

ISN 0006

ISN 0007

ISN 0008

ISN 0005

ISN 0010

ISA 0012

ISN 0014

ISN 0015

ISN 0016

ISN 0017

ISN 001 \&

ISN COIS

ISN 0020

ISA 0021

ISN -0022

ISA 0023

ISA 0024

ISN JO25

ISN 0025

ISA 0027

ISN 0028 SUBROUTINE PHSC (PHSAT, T, DHCCN,HSGAS. HSLIQI

IMPLICIT REAL $* \delta(A-Z)$

INTEGER I

DIMENSITN PSATI31), TSATI31), HCON(31), HSASI31)

DBS 42760 DBS42770 DES 42780 $D B \$ 42790$

DATA PSAT/7.5, 5.28,11.53,14.41,17.32,21.83,26.48,31.86,38.04,45.0608S42800 $1,52.58,61.55,72.04,83 \cdot 22,95.73 \cdot 109.72 \cdot 125 \cdot 18 \cdot 141.98 \cdot 160 \cdot 55 \cdot 180 \cdot 89 \cdot 08542810$ $2203.04,227.20,253.41,281.85,312.60,345.82,381.57,420.11,461.54,50508542820$ 3.E5,529.06/

DB 542830

DATA TSAT/ $-20,-10 \ldots 0 \ldots 10 \ldots 20 \ldots 30 \ldots 40 \ldots 50 \ldots 60 \ldots 70 \ldots 80 \ldots 90 \ldots 100 \ldots 110 B S 42840$

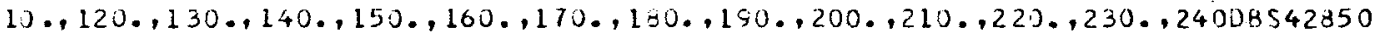
$2 ., 250 ., 260 ., 270 ., 274.961$ OB $\$ 42860$ DATA HCON/ 162.8.160.5.158.9,156.8,154.6,152.2,145.7,147.2,144.5,14DB542870 $11.9,139,1,130.1,133.1,130.1,127 ., 123.7,120.2,116.4,112.3,108,2,10308542880$ $2.8,98 ., 53.5,88.4,82.4,75.6,68.1 .55 .3 .48 .7 \cdot 33.9 \cdot 0.1$ DBS 42990 DATA HGAS/ $-688.4,-685.1,-681.8,-678.5,-675.2,-671.5,-668.7,-665.5,0 B S 42900$ $1-662.2,-659.1,-656.0,-652.9,-649.8,-646.7,-643.7,-640.6,-637.5,-63$ DBS 42910 $24.6,-631.8,-629.0 .-626.3 .-623.8,-621.4 .-619.0 \cdot-617.0,-615.6,-014.708 \$ 42920$ $3,-614 \cdot 2,-614 \cdot 5,-616.7,-631.21$

C IF TEMPERATURE IS OUTSIDE TABLE LIMITS SET TO TABLE LIMITS IFIT .LE. TSATILU T=TSAT(L)

IF(T . GE. TSAT(21)) T=TSAT(31)

DO $3 J \quad I=1,31$

I F(TSATII)-T) $30,10,20$

10 PHSAT = PSAT (I)

DHCCN $=$ HCON (I)

HSGAS =HGAS( I)

GC TO 40

$20 \times 1=(T-T S A T(I-1))((T S A T(I)-T S A T(I-1))$

PHS $A T=P S A T(I-1) \neq(1,-X 1)+X 1 * P S A T(1)$

OHCON $=H \operatorname{CON}(1-i) *(1,-X 1)+X 1 *+\operatorname{CON}(I)$

$H S G A S=+4 G A S(1-1) *(1,-x 1)+X 1 * H G A S(1)$

$G O$ TO 40

30 CONTINUE

40 HSL $1 Q=H S G A S-C H C O N$ RETURN

END
DBS 42930

08542940 $D B \subseteq 42950$

DBS42960 DB 542970 DBS4 4980 DBS 42990 OB 543000 DBS43010 DB 543020 DRS43030 DBS43040 DBS43050 DBS43060 DBS43070 DBS 43080 DB 543090 DA\$431J0 DAS 43110 
CCNPILER CPTICNS - NAME $=$ MAIN, DPT $=00$, LINECNT $=58, S I Z E=0000 \mathrm{~K}$,

ISN 0002

ISN 0003

ISA 0004

ISN 0005

ISN OOJ6

ISN 0007

ISN J.J08

ISN 0005

I SN 0010

ISN OJII

ISA OJI3

ISN OOIS

ISN OOIS

ISN COI7

ISN OUI8

ISN OUIS

ISN 0020

ISN 0021 SQUURCE, EBCCIC, ACLIST, NODECK, LOAD. MAP, NOEDIT , NOID, XREF FUNCTION UHEETRR (PRES, ENTH)

INPLICIT REAL $\$ 8(A-Z)$

INTECER IO,JOI,J,NPR,AEN

CCMMI]N/POIN/NPR, NEN, FI, FJ,IC,JO,I,J

DIMENSICN ENTR $(23,15)$, ENT $1(101)$, ENT2(161), ENT3(115)

EQUIVALENCE (ENTR(1,1). ENT1 (1)), (ENTR (1,8), ENT2(1), (ENTR(1,15), ENDBS43170

IT $3(1)$

FUNCTION TO FINU ENTRUPY FRCM PRESSURE AND ENTHALPY

DBS 43120

DB $\$ 43130$

$O B \subseteq 43140$

DBS 43150

DBS 43190

DATA ENTL/23*.816,.862,.860,.8858,.856,.855,.855,.856,.855, .857,.8808543200 $156, .856, .856, .857, .856, .8565, .856, .854, .853, .852, .8515, .850, .848, .08543210$ $2346, .508, .505, .5002, .8585, .897, .856, .8961, .854, .893, .893,6 \$ 892, .808543220$ $3915, .891, .850, .885, .887, .8855, .863, .952, .549, .943, .935, .9565, .93550 R S 43230$ $4, .933, .932, .931, .931,3 * .930,2 * .929,2 * .928, .927, .9265, .926, .924, .9208543240$ $51 \ldots 915,1.01, .593, .585, .980, .9765, .9745, .971 \ldots 969, .967, .967, .966, .908543250$ E6,.965,.967,.964,2*.96इ,.962,.961,.900,.958,.956,.951,1.05,1.037,1DBS43260 $7.028,1.022,1.0175,1.015,1.010,1.006,1.004,1.003,1.002 .1 .000, .999, .0 B 543270$ $8997,3 * .596, .9555, .555, .994, .952, .990, .981,1.09,1.081,1.070,1.063,108543280$ $9.058,1.054,1.049,1.044,1.041,1.036,1.037,1.035,1.054,1.050,1.029,108543290$ $1.028 \cdot 1.027 .1 .027 .1 .026 \cdot 1.025,1.023 .1 .020 .1 .0161$ DA $\$ 43300$ DATA ENT2/1.214,1.126,1.114,1.105,1.0\$8,1.093,1.087,1.081,1.077,1.08543310 $1 J 74,1.072,1.070,1.069,1.063,1.060,1.05,1.057,1.056,1.055,1.04,1.0 \mathrm{~S} \$ 43320$ $2 J 52,1.045,1.040 \cdot 1 \cdot 246 \cdot 1 \cdot 168 \cdot 1.155 \cdot 1 \cdot 145,1 \cdot 139,1 \cdot 134,1 \cdot 126,1 \cdot 120,1.08543330$ $3116,1.112,1.109,1.107,1.104,1.097,1.052,1.089,1.087,1.084,1.083,1.03543340$ $4082.1 .380,1.078,1.073,1.286,1.209,1.195,1.185,1.177,1.172,1.163 .1 .08543350$ $5157,1.152,1.147,1.145,1.140,1.138,1.130,1.124,1.119,1.117,1.113,1.08543360$ $6110,1.109,1.107,1.105,1.095,1.324,1.245,1.230,1.221,1.214,1.207,1.08 \$ 43370$ $7199,1.152 \cdot 1.187 \cdot 1 \cdot 182 \cdot 1 \cdot 178 \cdot 1 \cdot 176 \cdot 1 \cdot 171 \cdot 1 \cdot 160 \cdot 1 \cdot 155 \cdot 1 \cdot 149 \cdot 1 \cdot 146 \cdot 1 \cdot 0 B 543380$ $3141,1.138,1.136,1.133,1.131,1.122,1.358,1.279,1.266,1.256,1.249,1.0 R \$ 43390$ $9243,1.233,1.226,1.220,1.216,1.212,1.206,1.2 J 4,1.193,1.185,1.179,1.085434 J 0$ $1175,1.165,1.166,1.163,1.160,1.157,1.147,1.391,1.308,1.258,1.256,1.0 R 543410$ $2267,1.260,1.263,1.257,1.252,1.246,1.242,1.238,1.235,1.223,1.215,1$. DP $\$ 43420$ $3208,1.204,1.197,1.193,1.190,1.186,1.182,1.171,1.418,1.342,1.327 \cdot 1.09543430$ $4317,1.305,1.304,1.254,1.286,1.281,1.270,1.271,1.268,1.264,1.252,1.0 B S 43440$ $5243,1.236,1.232,1.225,1.219,1.216,1.211,1.236,1.1931$ $08 \$ 43450$

DATA ENT3/1.446.1.372,1.355,1.349,1.341,1.335.1.325.1.314.1.311.1.DBS43460 $1306.1 .301,1.257,1.251,1.279,1.269,1.264,1.258,1.250,1.245,1.241,1.0 B S 43470$ $2235,1.229,1.216,1.472,1.397,1.382,1.373,1.365,1.359,1.349,1.341,1.0 B S 43480$ $3335,1.330 \cdot 1.326,1.322,1.318,1.304 .1 .296,1.289 .1 .284,1.275,1.269,1$. DR S 43490 $4264,1.258,1.252,1.239,1.498,1.420,1.407,1.396,1.385,1.383,1.373,1.0 B 543500$ $5366,1.359,1.354,1.349,1.345,1.342,1.329,1.321,1.313,1.307,1.299,1.08543510$ $6292.1 .288,1.281,1.276,1.262,1.521,1.443,1.430,1.415,1.411,1.405,1.08543520$ $7355,1.388,1.381,1.376,1.371,1.367,1.365,1.352,1.341,1.335,1.330,1.08543530$ $8322,1.315,1.303 \cdot 1.253 \cdot 1.285,1.283,1.544,1.464 \cdot 1.452,1.440,1.433,1.0 B S 43540$ $5427,1.417,1.411,1.403,1.398,1.393,1.389,1.385,1.372,1.362,1.354,1.08 \$ 43550$ $1348,1.338,1.330,1.324,1.314,1.307,1.304 /$

$I F(I D$. EQ. $(N P R+1)) \quad I C=N P R$

I F (JO . EQ: (NEN+I)) JO=NEN

$D B \$ 43560$

$X I=E N T R(I, J)$

$X_{2}=E N T R,(I C, J)$

$X 3=E N T R(I, J C)$

$X 4=E N T R(I O, J O)$

UHENTR $=G R$ I C (FI, FJ, $\left.x_{1}, x_{2}, x_{3}, x_{4}\right)$

RETURN

END

DBS 43570

DB $\$ 43580$

$D B \$ 43590$

DB 543600

DB 543610

DB $\$ 43620$

$D B \$ 43630$

DBS43640

DB $\$ 43650^{\circ}$ 


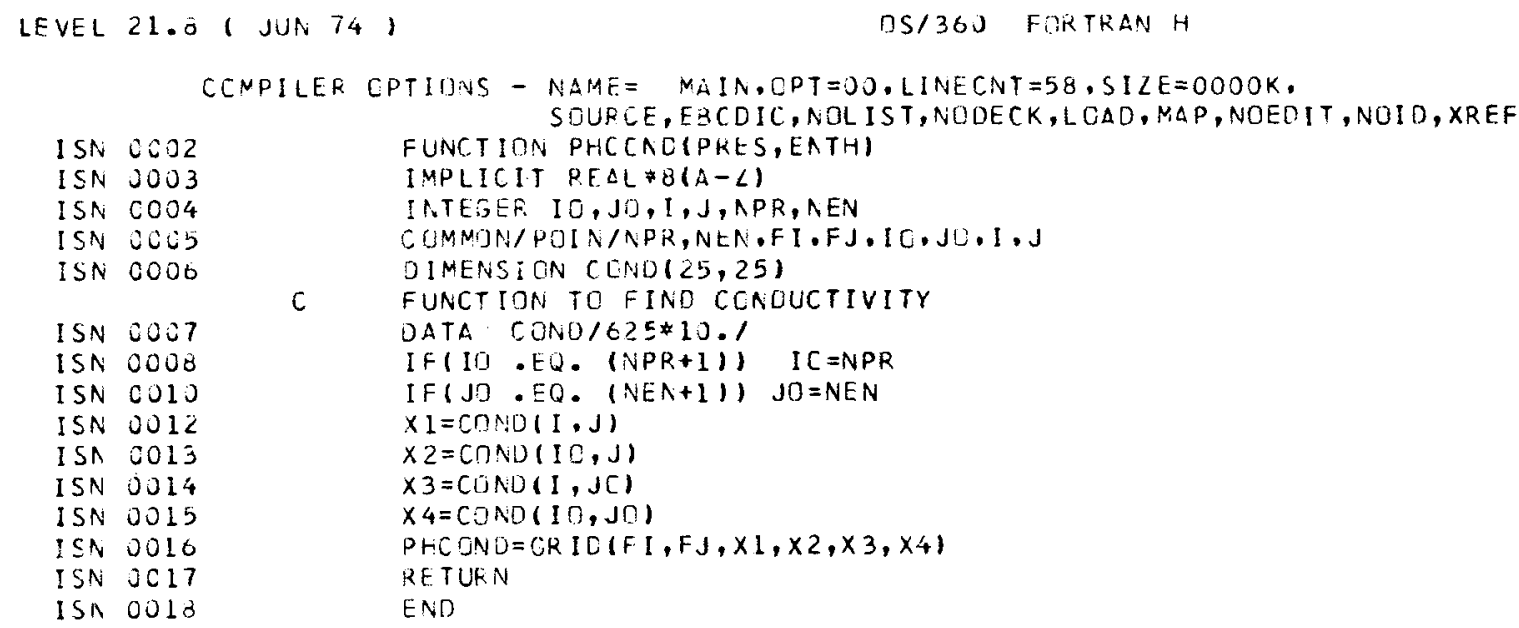

$1 \mathrm{SN} \operatorname{COO} 2$

ISN 3003

ISN $\mathrm{COO} 4$

ISN $0 \mathrm{COS}$

ISN COOB

ISN 0007

ISN 0008

ISN COIO

ISN 0012

ISA 0013

ISN 0014

ISN 0015

ISN 0010

ISN 3017

ISN $001 \%$

FUNCTION PHCCACIPKES, EATH)

IMPLICIT REAL $\#(A-\angle)$

INTEGEF. IO,JO,I,J,APR, AEN

CUMMON/ POIN/NPR,NEN,FI,FJ,IO,JU,I,J

DIMENSION CLND $(25,25)$

C FUNCTION TO FIND CCNDUCTIVITY

DATA CONO/EZE*10.1

IF(IO $E Q \cdot(N P R+1)) \quad I C=N P R$

$I F(J O \cdot E Q \cdot(N E N+1)) \quad J O=N E N$

$x I=\operatorname{Con} 0(I, \mathrm{~J})$

$x=\operatorname{COND}(I 0, j)$

$\times 3=\operatorname{CoND}(1, J()$

$x 4=\operatorname{CONO}(10, \mathrm{~J} \cap)$

PHCOND $=$ CRICIFI, FJ, XI, $\times 2, \times 3, \times 4)$

RETUKN

END

$08 \$ 43660$ DBS43670

DBS43680

$D 8 \$ 43690$

DRS $\$ 3700$

DBS43710

DRS43720

DBS $\$ 3730$

DBS43740

DBS 43750

DBS 43760

DRS 43770

DSS 43760

DBS 43790

DAS43800

UBS 43810 


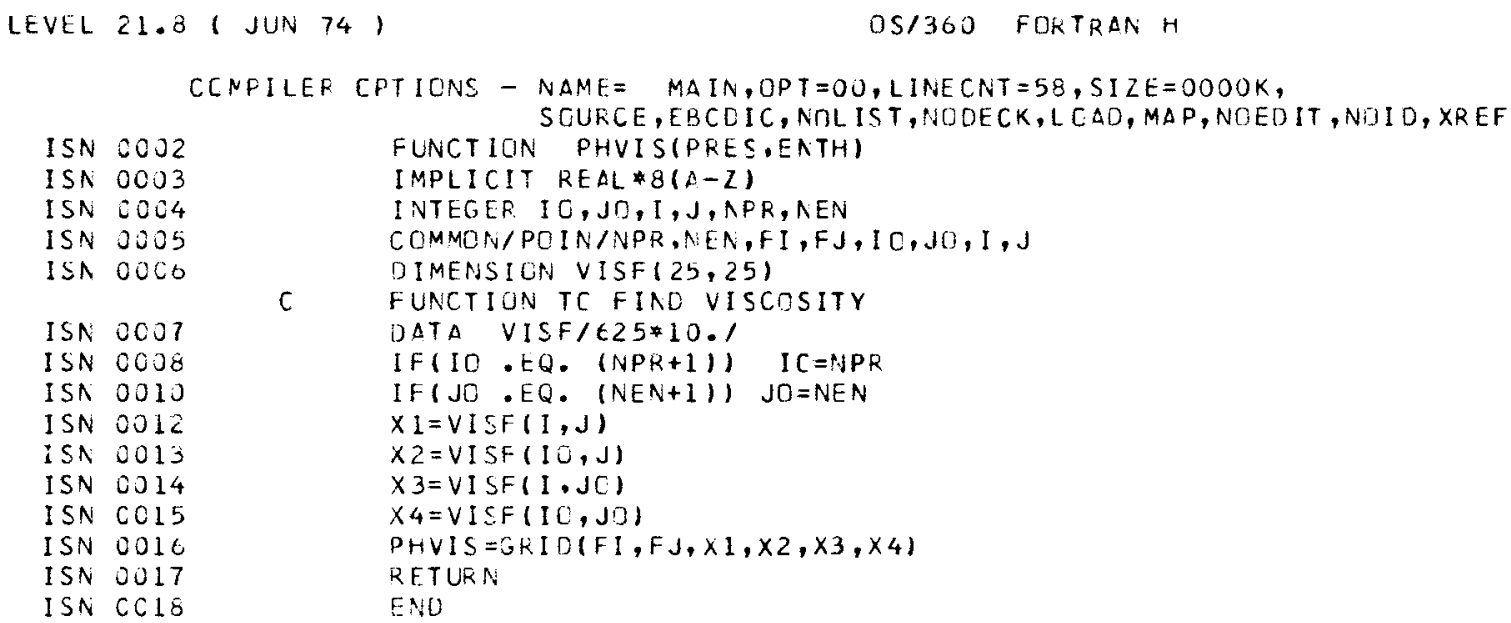

DBS 43820 DB\$43830 DBS43840 DBS 43850 DBS43860 DBS 43870

$D B \subseteq 43880$ DBS 43890 DR 543900 DBS $\$ 3910$ DBS 43920 DBS 43930 DBS 43940 DB $\$ 43950$ DBS43960 DBS 43970 


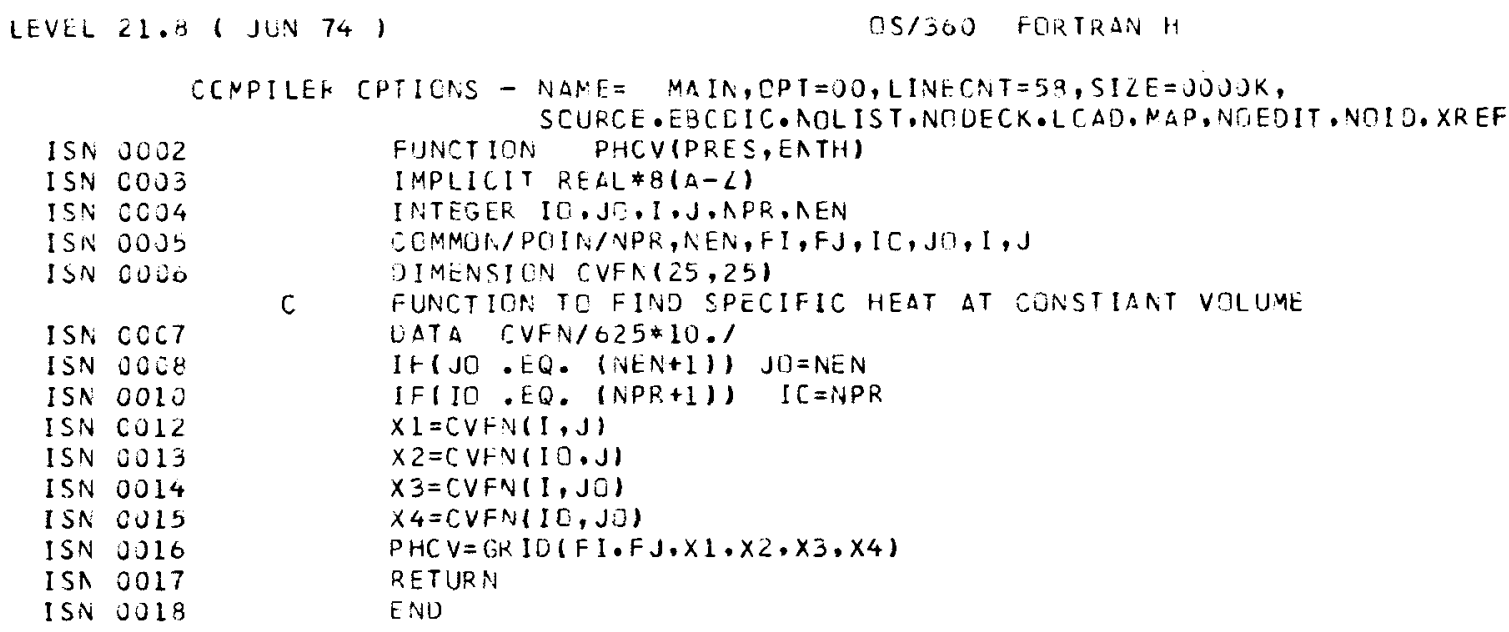

DB $\$ 43980$

DBS $\$ 3990$

DBS44000

DBS 44010

DBS44020

DBS 44030

DBS44040

DBS 44050

DRS44060

DB $\$ 44070$

DBS 44080

DB 544090

DBS 44100

DBS 44110

D. $\$ 44120$

DES 44130 
CCNPILER CPTIONS - NAME = MAIN, OPT=00, LINECNT=58, SILE= 0000K, SOURCE, EBCDIC, NOLIST, NODECK, LOAD, NAP, NOEDIT, NOID, XREF

ISN 0002

ISN 0003

ISN $30 C 4$

ISA 0005

ISN OUUÓ

ISN 0007
ISN 0008

ISN COOY

ISN 0011

ISA OUIZ

ISN 0013

ISA $\mathrm{CO} 14$

ISA 0015

ISN 0017

ISN 0018

ISN 0015

ISN 0021

ISA CO2S

ISN 0025

ISN 0026

ISN 0027

ISN 0028

ISN 0029

ISN 0031

ISN 0032

ISN 0033
SUBROUT INE ITER (I, X,YI,YJ,FLAG)

INPLICIT REAL $8(\&-2)$

INTEGER. I, J,ICLK

CCMMOIN/ITERS/ISWT (30)

CCMMIN/ITERSI/EPS(30), NINX(30), MAXX130), PCNT1 $(30)$, PCNTN(30). IICNT( 00$),$ ITF $(30)$, XNAMI 30$)$, YNAM 30$)$, MI, NI

CCMMON/ ITERS2, XOLO (30), YOLL(30), XMIN $(30), X M A X(30)$ )

C GENEPAL NEWTON TYPE I TERATION SCHEME

C CALL ITERII,X,YI,YJ, *I

I - ITEKATICN NUMBER

$X$ - INDEP VARIABLE

YI - DEP VARI $\triangle B L F F(X)$

YJ - UEP VARIAELE $G(X)$

ISWT - PRINT FLAG

OEL - DEP VARIABLE TOLERANCE

MIMX - MININUM $X$

MAXX - MAXINUM $X$

PCNTI - FRACTICAAL AMCUNT TO INCREMENT IST $X$

PCATN - HRACTIONAL AMOLNT TO INCRENENT NTH $X$

ICNT - COUNTER (1-15,NDT CLOSED) (J,CLOSEO)

ITF - FLAC, POS NNEWTCNS, NEG=COUNTEF TYPE ITERATION

XNAM - INOEP VARIARLE NAME( 8 CHAF MAX)

IIJQ = MON-CLDUSURF FLAG - - NON-CLOURE = NON-ZERI

YNAM - DEP VARIARLE NAME( 8 (HAH. MAX)

ITERATION LCCATEO IN STATEMEMT

NUMEER SUERCUTINE NUMBER

$1 \quad M A I N \quad 42$

$\therefore 1=9.00$

MAIN

43

IF(ICNTII).NE.O.) GO TO 10

C SAVE GRIGINAL MIN.NAX X ON IST PASS

$X M I N(I)=M I N X(I)$

$X M A X(1)=N \Delta X X(I)$

$10 \mathrm{DIFY}=Y I-Y J$

EPSY $=D A B S([I F Y / Y J)$

C CLUSURE TCLERANCL TEST

IFIEPSY.GT.EPSI I) IGO TC 20

ICNT(I) $=0$.

GE TO 80

c

CHECK FCR CLUNTER CNLY PATH

20 IFIICNTII).GE.NI) GO TC 100

IF(ITFII).LT.0.0) GC TC 70

IF(ICNT (I).NE. O.IGO TC 30

C IST PASS X INCREMENT CALCULATION

DELX = DSIGN(PCNTI(1),-DIFY)

GO TO 40

30 DIF $X=X-X$ CLDIII

DELY = YOLD(I) - DIFY

C NTH PASS X INCREMENT CALCULATION

IFIDELY.EQ.0.0I GC TO 36

DELX $=$ PCNTA(I) DIFX*DIFY/UELY

$L=X+D E L X$

IFIX.GI.Z.AND.YI.GT.YJ) GO TO 40
DBS44140

08544150

DBS 44160

DB 544170

DB 544180

DB 544150

UAS 44210

DES 44210

DBS 44220

DBS $\$ 4230$

DBS 44240

DB 544250

DB 544260

08544270

$D B \$ 44280$

DRS44290

DBS443JO

DBS 44310

DBS 44320

DR\$44330

DB 544340

DA 544350

DBS544360

DR $\$ 44370$

D 5544380

DBS 44390

DBS 44400

DHS 44410

$D B \$ 44420$

DBS 44430

DB $\$ 44440$

DBS44450

DBS44460

DB 544470

DSS44480

DBS 44490

DBS $445 J 0$

DRS44510

DBS 44520

DRS 5453 C

DBS44540

DR $\$ 4455$ ?

DBS 44560

DB 544570

DB\$44580

$D B \subseteq 44590$

DBS $\$ 4500$

DBS446́10

DBS44620

DBS 44630

DRS 44640

DES4465J

DB $\$ 44660$

DRS4467) 


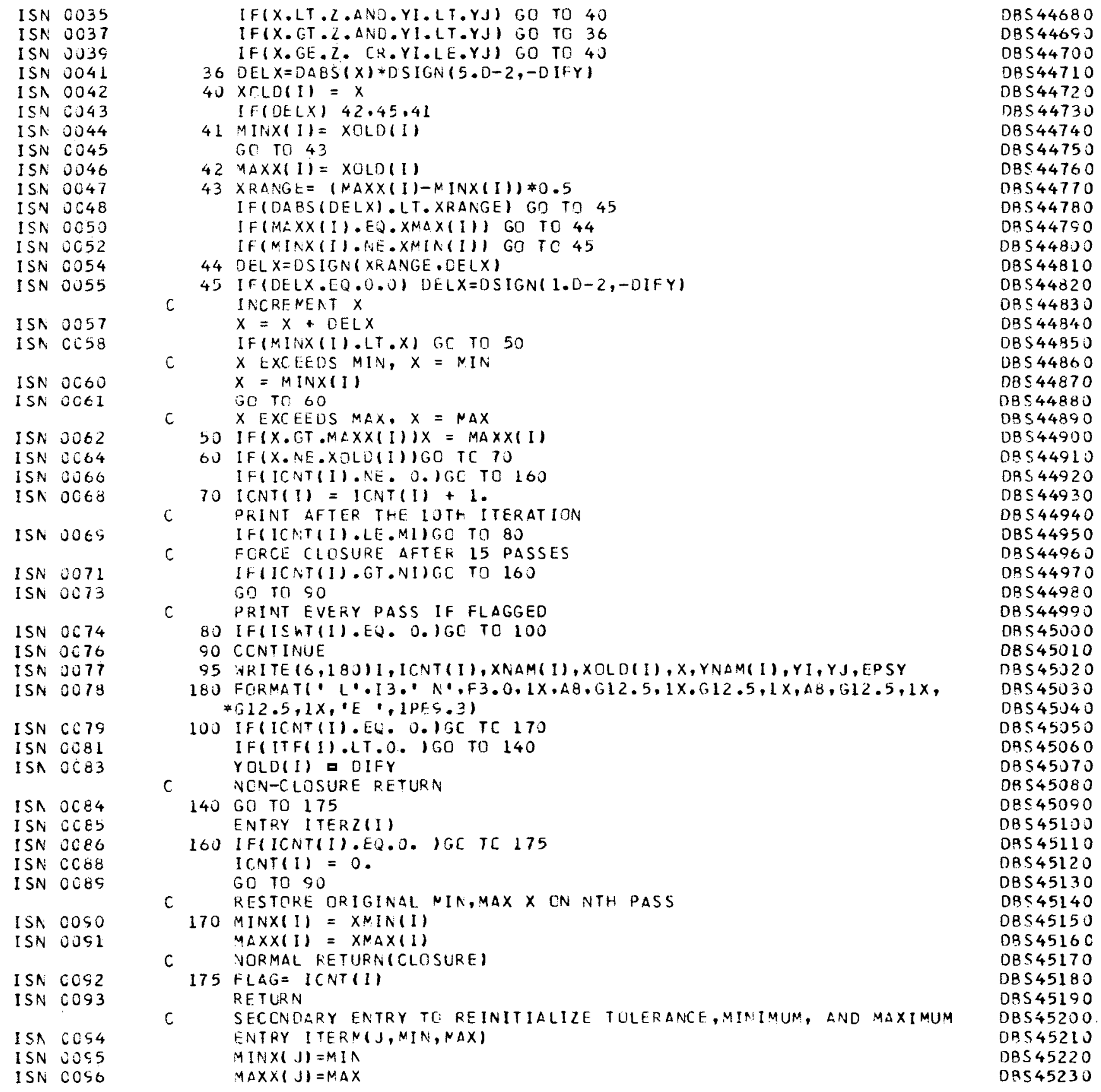

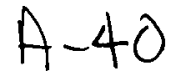


ISA 0057

ISN COS 8

RETURN

END
DBS45240

DBS 45250

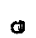

$A-4 \mid$ 


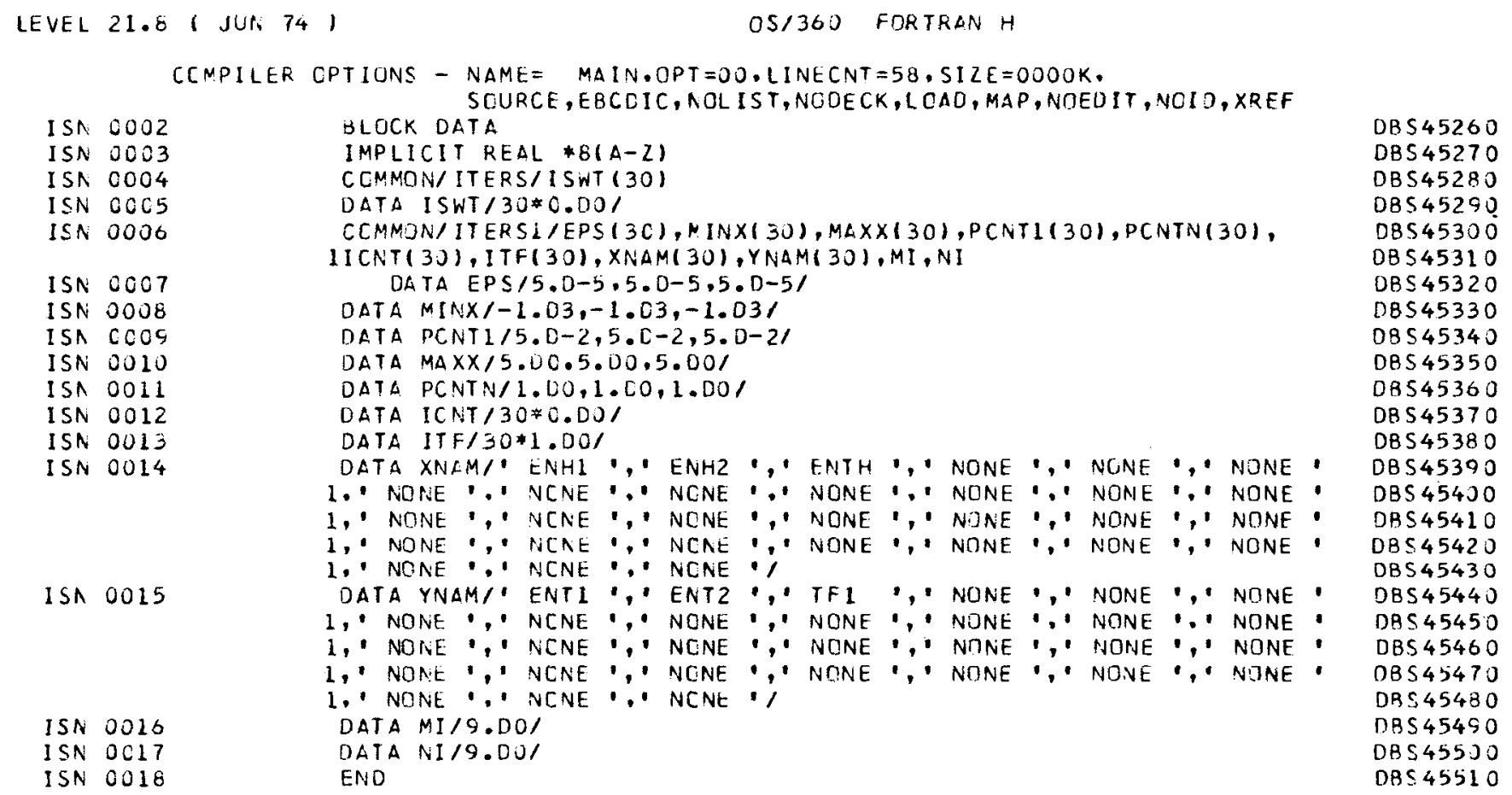




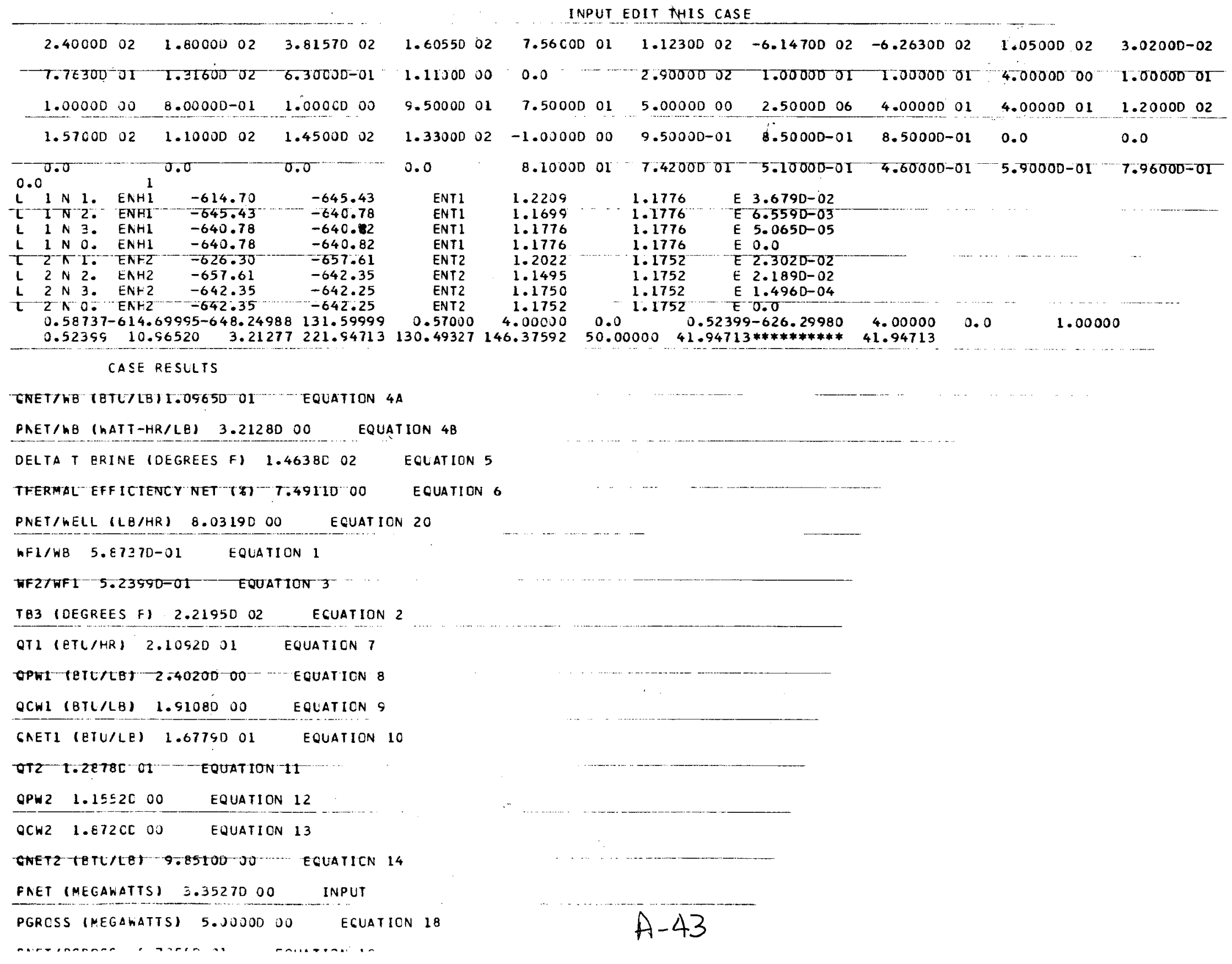




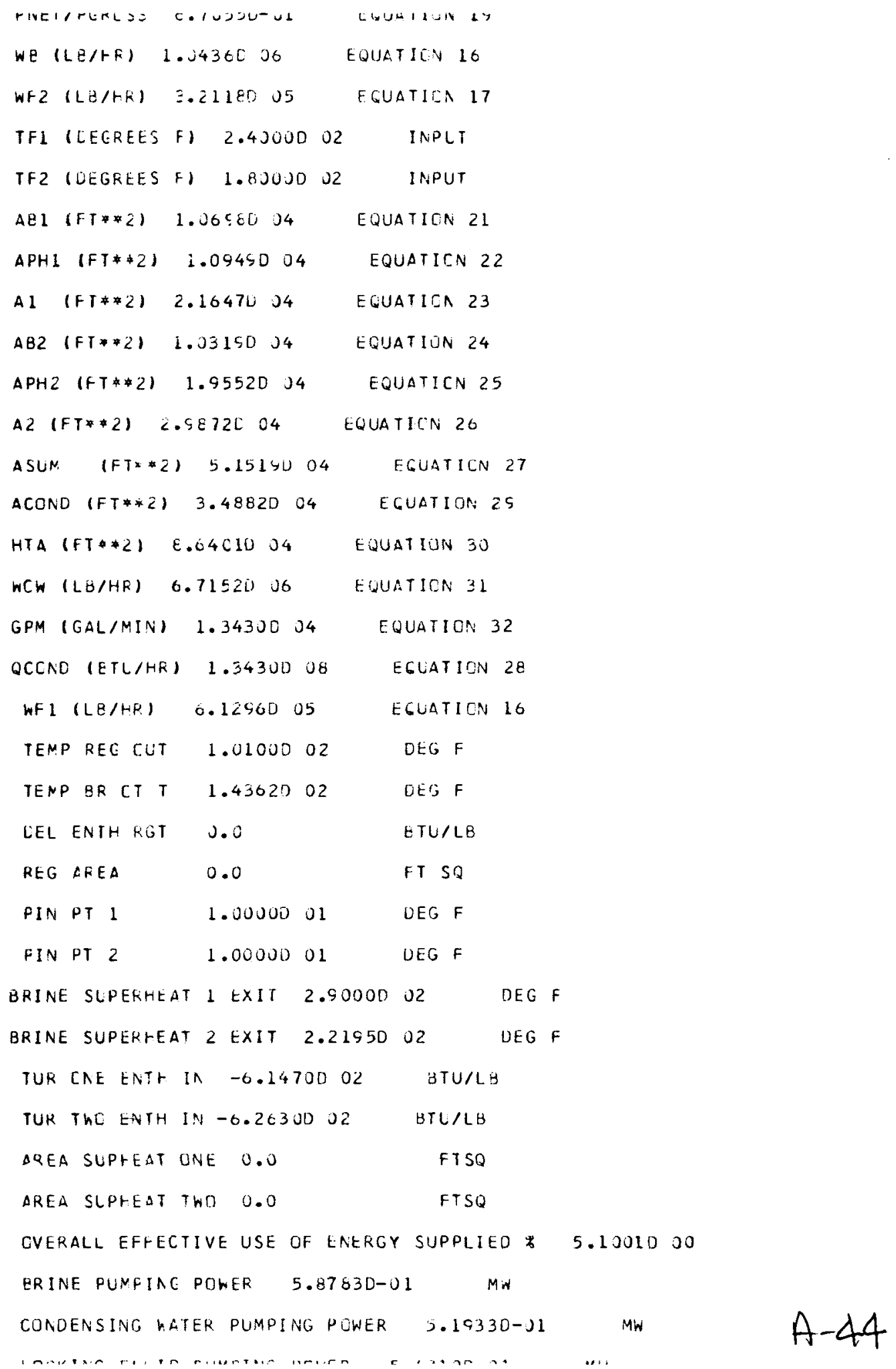




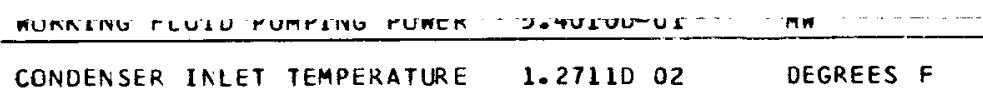

NUMBER CF ERINE SUPPLY WELLS 2

NUPEER OF ERINE REINJECTION WELLS I

NUMBER OF COOLING WATER SUPPLY WELLS 9

NUMB́ER CF CCCLING WATER REINJECTION WELLS

PRESSURE HP BCILER 3.81570 O2 PROP DECR

PRESSURE LP ECILER 2.0304002 PROP DECK

CCNDENSING PRESSURE 7.76300 OL PROP CECK

PUMP EFFTCIENCY $8.00000-01$ - INPUT

ELECTRICAL EFFICIENCY 9.5000D-01 INPUT

HP TURBINE EFFICIENCY $8.50000-01$ INPUT

LP TURBINE EFFICIENCY $8.50000=01$ INPUT

MAX FLOWRATE / BRINE SUPPLY WELL 2.50000 O6

SAT VAPOR ENTHALPY AT COND TEMP -6.48250 O2 TP PRDP DECK
TEMP BR INLET TP BR SPH 1 OT OT TP BR PRH 1 OT

TEMP BR INLET

TP TURI 03 O. $29000^{-103}$

0.2400003

EN TURI INLET $-0.61470 \quad 03$

P TUR2 TNL AREA BOILER I

AREA BOILER
0.10700 OS TUR2 INLET $-0.6263003$ $\triangle R E A$ BDILER

AREA SUPHET I AREA SUPHET 2 AREA BOILER 2

0.0
0.043210 SUPHET 2

0.2219003

TP BOILER ONE

TP BOILER TWO

0.1800003

AREA PREHET 1

0.1095005

DELT PIN PT 1

0.1000002

ELESCST TURGCST O.0 SECPCST

$\begin{array}{lllll}0.43270 \text { C6 } & 0.7549006 & 0.1212006\end{array}$

$0.1212006 \quad 0.6738 \mathrm{D} 06$

TP BR SPH 2 OT TP BR OUTLET BRINE FLOW RATE

FLOW RT TR ONE EN ACT EX TUR 1

FLOW RT TR THO EN EXT AOIL 2 EN ACT EX TUR2

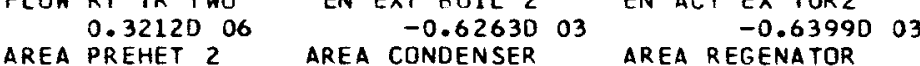

AREA PREHET 2

AREA CONDENSER

DELT PIN PT 2 TEMP REG OUT OU OS FLOW RATE COOL

0.7549006

0.6738006

COCTEST

0.9293006

0.1010003

$0.67150 \quad 07$

0.1340006

TOTSCST-CSTPOW MILS PR K K $0.1904006 \quad 0.5082007$

0.3076002 (Brine Flow Rate). 


\section{APPENDIX B \\ COST EQUATION \\ DERIVATIONS}




\section{COST EQUATION DERIVATIONS}

I. Total System Cost

A. Derivation

$$
\begin{aligned}
& \Phi_{T}=\left\{\Phi_{E}+\sum_{i} f_{i} \Phi_{E}\right\} F_{I}+\left\{\Phi_{W}+\sum_{W} f_{W} \Phi_{W}\right\} F_{I I} \\
& \text { where: }{ }^{\Phi}{ }_{T}=\text { total system cost } \\
& \Phi_{E}=\text { total equipment cost } \\
& \Phi_{W}=\text { supply system cost } \\
& \mathrm{F}_{\mathrm{I}}=\begin{array}{l}
\text { factor for Engineering, Design, and Inspection, } \\
\text { overhead and contingency }
\end{array} \\
& \begin{aligned}
F_{I I}= & \text { factor for exploration, land use, and exploratory } \\
& \text { drilling }
\end{aligned} \\
& f_{i}=\text { factors for piping, buildings, instrumentation, etc., } \\
& \text { and the remaining energy conversion cost } \\
& \mathrm{fw}=\text { factors for supply and disposal piping, pumps, } \\
& \text { insulation, etc., and energy supply cost }
\end{aligned}
$$

1. The total equipment cost, $\Phi_{E}$, is the sum of the major equipment costs of the energy conversion system:

$$
{ }_{E}{ }=\Phi_{H E}+\Phi_{C}+\Phi_{T-G}+\Phi_{P}+\Phi_{E S}
$$

where: a) ${ }^{\Phi} H E=$ heat exchanger cost

b) $\Phi_{E}=$ condenser and cooling tower cost

c) $\Phi_{T-G}=$ turbine-generator cost

d) $\Phi_{P}=$ secondary fluid pump cost

e) $\Phi_{E S}=$ electrical system cost

2. The supply system cost, $\Phi_{W}$, is the sum of the costs of drilling, casing, cementing, etc. 
B. Calculation (all areas in $\mathrm{ft}^{2}$, all flow rates in $1 \mathrm{bm} / \mathrm{hr}$, all power in $\mathrm{KW}$ )

1. $\Phi_{E}$, Total Equipment Cost

a) $\Phi_{H E}$, heat exchangers cost

$=\Phi$ BOILERS $+\Phi$ PREHEATERS

$=(10.56)$ (area of boilers) $+(6.91)$ (area of preheaters, regenerator, and superheaters)

b) $\Phi_{C}$, condenser and cooling tower cost

$=(7.6)$ (area of condenser) $+(24.25)$ (cooling water flow rate) 0.65

c) $\Phi_{T-G}$, turbine-generator cost

$=(8268.5)$ (gross power $)^{0.53}$

d) $\Phi_{p}$, secondary fluid pump cost

$=(2032$.$) (gross power )^{0.48}$

e) $\Phi_{E S}$, electrical system cost

$=(18,835)$ (gross power $)^{0.42}$

2. $\left\{\Phi_{W}+\sum_{W} f_{W} \Phi_{W}\right\} F_{I I}$, Total Supply System Cost

a) Based upon brine flow rate, $=(540$.$) (net power)$

b) Based upon the number of wells, $=(28,400$.$) (new power) (no. of wells)$

3. $\sum_{i} f_{i}=0.25$ and $F_{I}=1.34$, exclusive of contingency

4. Therefore, the Total System Cost is:

$$
\Phi_{T}=1.68 \Phi_{E}+540 \text { nnet power }(K W) \text { \} based upon brine flow rate }
$$

and

$$
\begin{gathered}
\Phi_{T}=1.68 \Phi_{E}+(28,400) \text { (no. of wells) (net power }(K W) \text { ) based upon } \\
\text { the number of wells } \\
B-\imath
\end{gathered}
$$




\section{Cost of Power}

Cost of Power $=$ $\left.\left[\frac{1}{\text { Net Power (KW) }}\right]\left[\frac{1000 \mathrm{mils}}{\mathrm{KW}-\mathrm{hr}}\right]\right\}\left[\frac{1 \mathrm{yr}}{(8760 \mathrm{hr} / \mathrm{yr})}\right]$

$\left.\left.\left[\frac{i(1+i)^{n}}{(1+i)^{n}-1}\right]\left[{ }_{E}+\sum_{i} f_{i} \Phi_{E}\right) F_{I}\right]+\left[\frac{i(1+i)^{n}}{(1+i)^{n}-1}\right]\left[\left(\Phi_{W}+\sum_{W} f_{W} \Phi_{W}\right) F_{I I}\right]\right\}$

Gathering Terms:

Cost of Power $=$

$\left(2.0454 \times 10^{-2}\right)\left(\Phi_{E}+\sum_{W} f_{i} \Phi_{E}\right) F_{I}+\left(2.2968 \times 10^{-2}\right)\left(\Phi_{W}+\sum_{W} f_{W} \Phi_{W}\right) F_{I I}$ Net Power Output (KW) 
Internal Distribution

1 - Chicago Patent Group - ERDA 9800 South Cass Avenue

Argonne, Illinois 60439

3 - A. T. Morphew Classification and Technical Information Officer ERDA - ID

Idaho Falls, Idaho 83401

1 - H. P. Pearson, Supervisor Technical Information

20 - Authors

35 - INEL Technical Library

External Distribution

573 - UC-66d - GE-UtiTization Technology, TID-4500, R65

Tota1 Copies Printed - 633 